\title{
A Rate-Distortion Approach to Caching
}

\author{
Roy Timo, Shirin Saeedi Bidokhti, Michèle Wigger and Bernhard C. Geiger
}

\begin{abstract}
This paper takes a rate-distortion approach to understanding the information-theoretic laws governing cache-aided communications systems. Specifically, we characterise the optimal tradeoffs between the delivery rate, cache capacity and reconstruction distortions for a single-user problem and some special cases of a two-user problem. Our analysis considers discrete memoryless sources, expected- and excess-distortion constraints, and separable and $\mathbf{f}$-separable distortion functions. We also establish a strong converse for separable-distortion functions, and we show that lossy versions of common information (Gács-Körner and Wyner) play an important role in caching. Finally, we illustrate and explicitly evaluate these laws for multivariate Gaussian sources and binary symmetric sources.
\end{abstract}

\section{INTRODUCTION}

$\mathbf{T}$ HIS paper takes a rate-distortion approach to understanding the information-theoretic laws governing cache-aided communications systems. To fix ideas, let us start by outlining some of the applications that motivated our study.

On-demand media streaming: Imagine an on-demand internet media provider, and consider the problem of streaming media to millions of users. A common problem is that the users will most likely request and stream media during periods of high network congestion. For example, most users would prefer to watch a movie during the evening, rather than during the early hours of the morning. Downloading bandwidth hungry media files during such periods leads to further congestion, high latency, and poor user experience.

To help overcome this problem, content providers often cache useful information about the media database in small storage systems at the network edge (with fast user connections) during periods of low network congestion. The basic idea is that information placed in these caches will not have to be transported later over a congested network. Naturally these small storage systems cannot host the entire media library, so the provider must carefully cache information that will be most useful to the users' future requests.

Distributed databases: Now imagine a large database that is distributed over a vast global disk-storage network. Such a database might contain measurements taken by weather or traffic sensors spread across several countries; the time-series prices of companies' stock (or, FX prices) at different exchanges; the shopping history of customers; the browsing history of users; or the mobility patterns (or, channel-state measurements) of mobile devices in cellular networks.

Now suppose that a user queries the database and requests an approximate copy of one file (or, perhaps, a function of several files). Since the database is large and distributed, we can expect that it will need to make several network calls to load relevant data in memory before it can communicate the file to the user. Such network calls are performance bottlenecks, potentially leading to high latency and network traffic costs.

Modern database systems handle such problems by smartly caching the most common queries in fast memory. If, for example, it is known in advance that the user will request the weather forecast of a particular city, then we can simply cache part or all of this forecast in memory. Obviously, however, we cannot always know in advance what data will be requested, so we should carefully cache information that is useful to many different requests.

The main purpose of this paper is to help develop a better understanding of such cache-aided communications systems. We will focus on single-user systems, and we will try to determine the "most useful" information to place in the cache.

In the spirit of Maddah-Ali and Niesen [1. 2], we will break the problem into two distinct phases: A caching phase concerning the pre-placement of information in the cache, and a delivery phase concerning the reliable communication of the particular source (or, file) requested by the user.

Since the caching phase occurs before the user makes its request, it seems reasonable that the information placed in the cache should be common to many different sources in the library. Moreover, to minimise overhead and latency during peak-congestion times, it seems reasonable that the delivery-phase message should not duplicate any information already stored in the cache. With this in mind, we will focus on the following problems. For a given library of sources and a given cache capacity:

- What "common information" should be put in the cache?

- What is the minimum delivery-phase rate needed to achieve a given fidelity requirement at the user?

R. Timo is with Ericsson Research, Stockholm, roy.timo@ericsson.com. S. Saeedi Bidokhti is with the Department of Electrical Engineering, Stanford University, saeedi@stanford.edu. M. Wigger is with the Communications and Electronics Dsepartment, Telecom ParisTech, michele.wigger@telecomparistech.fr. B. Geiger is with the Institute for Communications Engineering, Technical University of Munich, bernhard.geiger@tum.de.

Some of the material in this paper was completed by R. Timo at the Technical University of Munich and presented at the International Zurich Seminar on Communications (IZS), March, 2016.

S. Saeedi Bidokhti was supported by the Swiss National Science Foundation Fellowship no. 158487. Bernhard C. Geiger was supported by the Erwin Schrödinger Fellowship J 3765 of the Austrian Science Fund. 
Our study will make the following specific assumptions.

- The library consists of $L$ different sources, and each source consists of $n$ symbols. Here $L$ is any fixed positive integer, and we consider the information-theoretic limits of cache-aided communications in the limit $n \rightarrow \infty$.

- The cache can reliably store up to $n C$ bits, and it is said to have capacity $C$.

- The fidelity of the user's reconstruction of the requested source can be meaningfully measured by a separable distortion function or, more generally, by an $\mathbf{f}$-separable distortion function 1 .

- The $L$ sources are generated by an arbitrary $L$-component discrete memoryless source (DMS). This assumption is quite common in the multi-terminal information theory literature, admits rigorous proofs and nevertheless gives a great deal of insight about more complicated models. Although it seems restrictive, some important transformations (e.g. BurrowsWheeler) are known to emit almost memoryless processes [5, 6].

Our paper is most related to Wang, Lim and Gastpar [7]. A key difference to [7], however, is the source request model: Wang et al. assumed that each user randomly selects a symbol from each source at each time in an independent and identically distributed (iid) manner. They then leveraged connections to several classic multi-terminal problems to establishe some interesting tradeoffs between the optimal compression rate and cache capacity under a lossless ${ }^{2}$ reconstruction constraint. In contrast to [7], we will require that the user requests one source in its entirety, we do not place prior probabilities on the user's selection, and we allow for lossy reconstructions. We thus consider a lossy worst-demand (i.e., compound source) scenario, while [7] considered an ergodic iid-demand scenario.

Hassanzadeh, Erkip, Llorca and Tulino $[8]$ recently studied cache-aided communications systems for transmitting independent memoryless Gaussian sources under mean-squared error distortion constraints. Their caching schemes exploited successiverefinement techniques to minimise the mean-squared error of the users' reconstructions, and they presented a useful "reverse filling-type solution" to the minimum distortion problem. Yang and Gündüz [9] consider the same cache-aided Gaussian problem, but instead focussed on the minimum delivery-phase rate for a given distortion requirement. They presented a numerical method to determine the minimum delivery rate, and proposed two efficient caching algorithms.

This paper will try to broaden the above work and improve our understanding of cache-aided communications. We will do this by, for example, considering arbitrarily correlated sources and lossy reconstructions with respect the general class of f-separable distortion functions.

Organisation: The problem setup is formally described in Section III Our main results for separable distortion functions are presented in Sections III to VI Specifically, Section III presents the optimal rate-distortion-cache tradeoffs; Section IV shows that two information-theoretic notions of common information (Gács-Körner and Wyner) play an important role in caching; Section Vrelates caching to Gray and Wyner's seminal paper "Source coding for a simple network" [14]; Section VI presents a strong converse (in the sense of Kieffer [15]); and Section VII considers Gaussian and binary sources. Finally, Section VIII presents the optimal rate-distortion-cache tradeoffs for $\mathbf{f}$-separable distortion functions, and Section IX considers a two-user version of the problem.

\section{Problem Setup}

\section{A. Source Model and RDC Codes}

Let $\mathcal{L}=\{1, \ldots, L\}$ denote the set of indices corresponding to the $L$ sources in the library. We assume throughout that the $\ell$-th source consists of $n$ symbols,

$$
X_{\ell}^{n}=\left(X_{\ell, 1}, X_{\ell, 2}, \ldots, X_{\ell, n}\right),
$$

with each drawn from a finite alphabet $\mathcal{X}_{\ell}$. The $L$ sources

$$
\boldsymbol{X}^{n}=\left(X_{1}^{n}, X_{2}^{n}, \ldots, X_{L}^{n}\right)
$$

are randomly generated by a $L$-component discrete memoryless source $(D M S)$; that is, $\boldsymbol{X}^{n}$ is a sequence of $n$ independent and identically distributed (iid) tuples $\boldsymbol{X}=\left(X_{1}, \ldots, X_{L}\right)$ characterized by an arbitrary joint pmf $p_{\boldsymbol{X}}(\boldsymbol{x})$ defined on the Cartesian product space $\mathcal{X}=\mathcal{X}_{1} \times \cdots \times \mathcal{X}_{L}$. Let $\hat{\mathcal{X}}_{\ell}$ be an appropriate finite reconstruction alphabet for the $\ell$-th source at the receiver and let $\hat{\mathcal{X}}=\hat{\mathcal{X}}_{1} \times \cdots \times \hat{\mathcal{X}}_{L}$.

A joint rate-distortion-cache $(R D C)$ code for a given blocklength $n$ is a collection of $(2 L+1)$ mappings:

(i) A cache-phase encoder at the server

$$
\phi_{\mathrm{c}}^{(n)}: \mathcal{X}^{n} \rightarrow \mathcal{M}_{\mathrm{c}}^{(n)} .
$$

Here $\mathcal{M}_{\mathrm{c}}^{(n)}$ is a finite (index) set with an appropriate cardinality for the cache capacity.

\footnotetext{
${ }^{1}$ Roughly speaking, separable distortion functions can be expressed as an average of a given per-letter distortion function over the sequence of pairs of source and reconstruction symbols. In $\mathbf{f}$-separable distortion functions this average is replaced by a more general function. Precise definitions are given in Definitions 1 and 2 in Section II-B The class of $\mathbf{f}$-separable distortion functions was recently introduced by Shkel and Verdú [3], and it has a rather appealing axiomatic motivation that we review later in Section II

${ }^{2}$ Specifically, Wang et al. required that a function of the source is reliably reconstructed (otherwise known as a deterministic distortion function).
} 


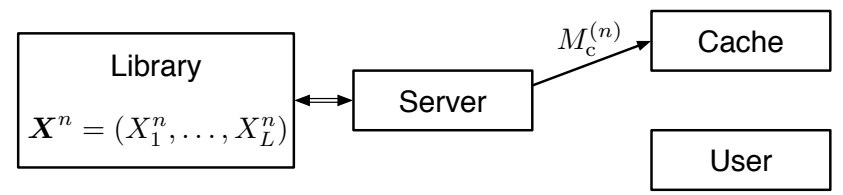

\section{Caching Phase}

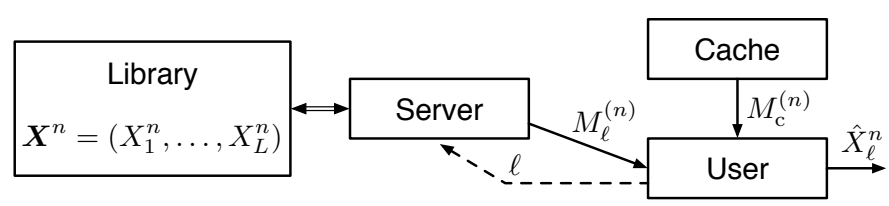

\section{Delivery Phase}

Fig. 1. A cache-aided communications system with a single user.

(ii) A delivery-phase encoder at the server

$$
\phi_{\ell}^{(n)}: \mathcal{X}^{n} \rightarrow \mathcal{M}^{(n)}
$$

for each user request $\ell \in \mathcal{L}$. Here $\mathcal{M}^{(n)}$ is a finite (index) set with an appropriate cardinality for the delivery phase.

(iii) A delivery-phase decoder at the user

$$
\varphi_{\ell}^{(n)}: \mathcal{M}^{(n)} \times \mathcal{M}_{\mathrm{c}}^{(n)} \rightarrow \hat{\mathcal{X}}_{\ell}^{n}
$$

for each possible user request $\ell \in \mathcal{L}$.

We call the above collection of encoders and decoders an $\left(n, \mathcal{M}^{(n)}, \mathcal{M}_{\mathrm{c}}^{(n)}\right)$-code.

During the caching phase (before the user requests a particular source), the server places the message $M_{\mathrm{c}}^{(n)}=\phi_{\mathrm{c}}^{(n)}\left(\boldsymbol{X}^{n}\right)$ in the cache. At some later time (the delivery phase), the user picks $\ell \in \mathcal{L}$ arbitrarily and requests the corresponding source $X_{\ell}^{n}$ from the server. The server responds to the user's request with the message $M_{\ell}^{(n)}=\phi_{\ell}^{(n)}\left(\boldsymbol{X}^{n}\right)$, and the user attempts to reconstruct $X_{\ell}^{n}$ by computing $\hat{X}_{\ell}^{n}=\varphi_{\ell}^{(n)}\left(M_{\ell}^{(n)}, M_{\mathrm{c}}^{(n)}\right)$. This cache-aided encoding and decoding process is illustrated in Figure 1.

A key point to note here is that the cache message $M_{\mathrm{c}}^{(n)}$ is encoded and stored in the cache before the user selects $\ell \in \mathcal{L}$ and requests the corresponding source $X_{\ell}^{n}$ from the server. Hence, the cached message $M_{\mathrm{c}}^{(n)}$ should (in a sense to be made precise later) efficiently represent useful common information shared between sources in the library.

\section{B. Separable and $\mathbf{f}$-Separable Distortion Functions}

As per the usual RD paradigm, let us first assume that the fidelity of the user's reconstruction $\hat{X}_{\ell}^{n}$ of the $\ell$-th source $X_{\ell}^{n}$ can be meaningfully quantified by the arithmetic mean of single-symbol distortions. Specifically, for each $\ell \in \mathcal{L}$ let

$$
\mathrm{d}_{\ell}: \hat{\mathcal{X}}_{\ell} \times \mathcal{X}_{\ell} \rightarrow[0, \infty)
$$

be a single-symbol distortion function. For example, we will often take $\mathrm{d}_{\ell}$ to be the Hamming distortion function where $\hat{\mathcal{X}}_{\ell}=\mathcal{X}_{\ell}$ and

$$
\mathrm{d}_{\ell}\left(\hat{x}_{\ell}, x_{\ell}\right)= \begin{cases}1 & \text { if } \hat{x}_{\ell} \neq x_{\ell} \\ 0 & \text { if } \hat{x}_{\ell}=x_{\ell} .\end{cases}
$$

We assume throughout that each $\mathrm{d}_{\ell}$ satisfies the following two conditions:

- For each source symbol $x_{\ell} \in \mathcal{X}_{\ell}$ there exists a reconstruction symbol $\hat{x}_{\ell} \in \hat{\mathcal{X}}_{\ell}$ such that $\mathrm{d}_{\ell}\left(\hat{x}_{\ell}, x_{\ell}\right)=0$.

- There exists a finite $D_{\max }>0$ such that $\mathrm{d}_{\ell}\left(\hat{x}_{\ell}, x_{\ell}\right) \leq D_{\max }$ for all $x_{\ell} \in \mathcal{X}_{\ell}$ and $\hat{x}_{\ell} \in \hat{\mathcal{X}}_{\ell}$.

Definition 1 (Separable distortion function). The $n$-symbol distortion between a particular source realisation $x_{\ell}^{n} \in \mathcal{X}_{\ell}^{n}$ and reconstruction $\hat{x}_{\ell}^{n} \in \hat{\mathcal{X}}_{\ell}^{n}$ is

$$
\overline{\mathrm{d}}_{\ell}\left(\hat{x}_{\ell}^{n}, x_{\ell}^{n}\right):=\frac{1}{n} \sum_{i=1}^{n} \mathrm{~d}_{\ell}\left(\hat{x}_{\ell, i}, x_{\ell, i}\right) .
$$

Let $\mathbf{d}=\left(\mathrm{d}_{1}, \ldots, \mathrm{d}_{L}\right)$ and $\overline{\mathbf{d}}=\left(\overline{\mathrm{d}}_{1}, \ldots, \overline{\mathrm{d}}_{L}\right)$. 
Although separable distortion functions are almost ubiquitous in the literature, it is also useful to consider the broader class of $\mathbf{f}$-separable distortion functions recently introduced by Shkel and Verdú [3]. Specifically, let us define the following for each request $\ell \in \mathcal{L}:$ Let

$$
\mathrm{f}_{\ell}:[0, \infty) \rightarrow[0, \infty)
$$

be continuous and strictly increasing, and let $\mathrm{d}_{\ell}$ be a single-symbol distortion function (1).

Definition 2 (f-Separable distortion function). The $n$ symbol distortion between $x_{\ell}^{n} \in \mathcal{X}_{\ell}^{n}$ and $\hat{x}_{\ell}^{n} \in \hat{\mathcal{X}}_{\ell}^{n}$ is

$$
\overline{\mathrm{fd}}_{\ell}\left(\hat{x}^{n}, x^{n}\right):=\mathrm{f}_{\ell}{ }^{-1}\left(\frac{1}{n} \sum_{i=1}^{n} \mathrm{f}_{\ell}\left(\mathrm{d}_{\ell}\left(\hat{x}_{i}, x_{i}\right)\right)\right) .
$$

Let $\overline{\mathbf{f d}}=\left(\overline{\mathrm{fd}}_{1}, \ldots, \overline{\mathrm{fd}}_{L}\right)$.

The basic idea in (4) is to choose the function $f_{\ell}$ to assign appropriate (possibly non-linear) "frequency costs" to different quantisation error events. If $\mathrm{f}_{\ell}$ is the identity mapping, then $\overline{\mathrm{fd}}_{\ell}$ reduces to the usual separable distortion function $\overline{\mathrm{d}}_{\ell}$ generated by $\mathrm{d}_{\ell}$. Several interesting connections between $\mathrm{f}$-separable distortions and Rényi entropy, compression with linear costs, and subadditive distortion functions are discussed in [3]. Moreover, $f$-separable distortions have a rather pleasing axiomatic motivation based on the following observation by Kolmogorov [4].

Proposition 1. Let $\left\{a_{1}, \ldots, a_{n}\right\}$ be a set of $n$ real numbers and $\overline{\mathrm{M}}_{n}: \mathbb{R}^{n} \rightarrow \mathbb{R}$ satisfy the following four axioms of mean:

(i) $\overline{\mathrm{M}}_{n}\left(a_{1}, \ldots, a_{n}\right)$ is a continuous and strictly increasing function of each argument $a_{i}$.

(ii) $\overline{\mathrm{M}}_{n}\left(a_{1}, \ldots, a_{n}\right)$ is a symmetric function of its arguments.

(iii) $\overline{\mathrm{M}}_{n}(a, \ldots, a)=a$.

(iv) For any integer $m \leq n$,

$$
\overline{\mathrm{M}}_{n}\left(a_{1}, \ldots, a_{m}, \ldots, a_{n}\right)=\overline{\mathrm{M}}_{n}\left(a, \ldots, a, a_{m+1}, \ldots, a_{n}\right),
$$

where $a=\overline{\mathrm{M}}_{m}\left(a_{1}, \ldots, a_{m}\right)$.

Then $\overline{\mathrm{M}}_{n}$ must take the form [4. p. 144]

$$
\overline{\mathrm{M}}_{n}\left(a_{1}, \ldots, a_{n}\right)=\mathrm{f}^{-1}\left(\frac{1}{n} \sum_{i=1}^{n} \mathrm{f}\left(a_{i}\right)\right)
$$

for some continuous and strictly increasing $\mathrm{f}$.

\section{Operational RDC Functions}

We will consider two different problem formulations: Optimal caching subject to an expected distortions criteria, and optimal caching subject to an excess distortions criteria. Throughout, let $\boldsymbol{D}=\left(D_{1}, \ldots, D_{L}\right)$.

Definition 3. We say that a rate-distortion-cache tuple $(R, D, C)$ is $\overline{\mathbf{f d}}$-achievable with respect to (w.r.t.) expected distortions if there exists a sequence of $\left(n, \mathcal{M}^{(n)}, \mathcal{M}_{c}^{(n)}\right)$-codes such that

$$
\begin{aligned}
& \limsup _{n \rightarrow \infty} \frac{1}{n} \log \left|\mathcal{M}_{c}^{(n)}\right| \leq C, \\
& \limsup _{n \rightarrow \infty} \frac{1}{n} \log \left|\mathcal{M}^{(n)}\right| \leq R, \text { and } \\
& \limsup _{n \rightarrow \infty} \mathbb{E}\left[\overline{\mathrm{fd}}_{\ell}\left(\hat{X}_{\ell}^{n}, X_{\ell}^{n}\right)\right] \leq D_{\ell}, \quad \forall \ell \in \mathcal{L} .
\end{aligned}
$$

The RDC function w.r.t. expected distortions $\mathrm{R}_{\mathbf{f}}^{\dagger}(D, C)$ is the infimum of all rates $R \geq 0$ such that the rate-distortion-cache tuple $(R, \boldsymbol{D}, C)$ is $\overline{\mathbf{f d}}$-achievable.

Definition 4. We say that a rate-distortion-cache tuple $(R, D, C)$ is $\overline{\mathbf{f d}}$-achievable w.r.t. excess distortions if there exists a sequence of $\left(n, \mathcal{M}^{(n)}, \mathcal{M}_{c}^{(n)}\right)$-codes such that (5a) and (5b) hold and

$$
\lim _{n \rightarrow \infty} \mathbb{P}\left[\bigcup_{\ell \in \mathcal{L}}\left\{\overline{\mathrm{fd}}_{\ell}\left(\hat{X}_{\ell}^{n}, X_{\ell}^{n}\right) \geq D_{\ell}\right\}\right]=0 .
$$

The RDC function w.r.t. excess distortions $\mathrm{R}_{\mathbf{f}}^{\ddagger}(D, C)$ is the infimum of all rates $R \geq 0$ such that the rate-distortion-cache tuple $(R, \boldsymbol{D}, C)$ is $\overline{\mathbf{f d}}$-achievable.

For separable distortion functions, we will omit the subscript $\mathbf{f}$ from the above definitions (because it is an identity mapping) and simply write $\mathrm{R}^{\dagger}(\boldsymbol{D}, C)$ and $\mathrm{R}^{\ddagger}(\boldsymbol{D}, C)$. 


\section{RESUlt: RDC FunCtion FOR SEPARABle Distortion FunCtions}

This section presents a single-letter expression (the informational RDC function) for the expected and excess operational RDC functions defined in Section $\Pi$

\section{A. Preliminaries}

We will need the following basic RD functions. The standard informational RD function of the $\ell$-th source $X_{\ell}$ w.r.t. $\mathrm{d}_{\ell}$ is

$$
\mathrm{R}_{X_{\ell}}\left(D_{\ell}\right):=\min _{p_{\hat{X}_{\ell} \mid X_{\ell}}: \mathbb{E}\left[\mathrm{d}_{\ell}\left(\hat{X}_{\ell}, X_{\ell}\right)\right] \leq D_{\ell}} I\left(X_{\ell} ; \hat{X}_{\ell}\right),
$$

where the minimisation is over all test channels $p_{\hat{X}_{\ell} \mid X_{\ell}}$ from $\mathcal{X}_{\ell}$ to $\hat{\mathcal{X}}_{\ell}$ satisfying the indicated distortion constraint.

The informational joint RD function of $\boldsymbol{X}$ w.r.t. $\mathbf{d}$ is [10]

$$
\mathrm{R}_{\boldsymbol{X}}(\boldsymbol{D}):=\min _{p_{\hat{\boldsymbol{X}} \mid \boldsymbol{X}}: \mathbb{E}\left[\mathrm{d}_{\ell}\left(\hat{X}_{\ell}, X_{\ell}\right)\right] \leq D_{\ell}, \forall \ell \in \mathcal{L}} I(\boldsymbol{X} ; \hat{\boldsymbol{X}}),
$$

where the minimisation is over all test channels $p_{\hat{\boldsymbol{X}} \mid \boldsymbol{X}}$ from $\mathcal{X}$ to $\hat{\mathcal{X}}$ satisfying all $L$ of the indicated distortion constraints.

The informational conditional RD function [10] of $X_{\ell}$ with side information $U$ is

$$
\mathrm{R}_{X_{\ell} \mid U}\left(D_{\ell}\right):=\min _{p_{\hat{X}_{\ell} \mid X_{\ell} U}: \mathbb{E}\left[\mathrm{d}_{\ell}\left(\hat{X}_{\ell}, X_{\ell}\right)\right] \leq D_{\ell}} I\left(X_{\ell} ; \hat{X}_{\ell} \mid U\right),
$$

where the minimisation is over all test channels $p_{\hat{X}_{\ell} \mid X_{\ell} U}$ from $\mathcal{X}_{\ell} \times \mathcal{U}$ to $\hat{\mathcal{X}}_{\ell}$ satisfying the indicated distortion constraint. The above minima exist by the continuity of Shannon's information measures, the bounded single-symbol distortion functions $\mathbf{d}$, and the fact that each (conditional) mutual information is minimised over a compact set.

\section{B. Result}

The next lemma summarises some basic properties of the operational RDC functions that we will use frequently. We omit the proof.

Lemma 2. The following statements are true for separable distortion functions.

(i) $\mathrm{R}^{\dagger}(\boldsymbol{D}, C)$ and $\mathrm{R}^{\ddagger}(\boldsymbol{D}, C)$ are convex, non-increasing and continuous in $(\boldsymbol{D}, C) \in[0, \infty)^{L+1}$.

(ii) If $C>\mathrm{R}_{\boldsymbol{X}}(\boldsymbol{D})$, then

$$
\mathrm{R}^{\dagger}(D, C)=\mathrm{R}^{\ddagger}(D, C)=0
$$

(iii) If $C=0$, then

$$
\mathrm{R}^{\dagger}(\boldsymbol{D}, 0)=\mathrm{R}^{\ddagger}(\boldsymbol{D}, 0)=\max _{\ell \in \mathcal{L}} \mathrm{R}_{X_{\ell}}\left(D_{\ell}\right) .
$$

(iv) If an $\left(n, \mathcal{M}^{(n)}, \mathcal{M}_{c}^{(n)}\right)$-code satisfies

$$
\mathbb{P}\left[\bigcup_{\ell \in \mathcal{L}}\left\{\overline{\mathrm{d}}_{\ell}\left(\hat{X}_{\ell}^{n}, X_{\ell}^{n}\right) \geq D_{\ell}\right\}\right] \leq \epsilon
$$

for some $\epsilon>0$, then the same code also satisfies

$$
\mathbb{E}\left[\overline{\mathrm{d}}_{\ell}\left(\hat{X}_{\ell}^{n}, X_{\ell}^{n}\right)\right] \leq D_{\ell}+\epsilon D_{\max }, \quad \forall \ell \in \mathcal{L} .
$$

We now define the informational RDC function of interest to our caching problem. Let $\mathcal{U}$ be a finite alphabet of cardinality $|\mathcal{U}| \leq|\mathcal{X}|+2 L$. The informational RDC function of interest is

$$
\mathrm{R}(\boldsymbol{D}, C):=\min _{U: I(\boldsymbol{X} ; U) \leq C} \max _{\ell \in \mathcal{L}} \mathrm{R}_{X_{\ell} \mid U}\left(D_{\ell}\right)
$$

where the minimisation is taken over the set of all auxiliary random variables $U$ on $\mathcal{U}$ jointly distributed with $\boldsymbol{X}$ satisfying the indicated mutual information constraint.

Theorem 3. For separable distortion functions, the RDC functions for both expected and excess distortions coincide with the informational RDC function in (7):

$$
\mathrm{R}^{\dagger}(\boldsymbol{D}, C)=\mathrm{R}^{\ddagger}(\boldsymbol{D}, C)=\mathrm{R}(\boldsymbol{D}, C) .
$$

Proof: Theorem 3 is proved in Appendix A.

The next corollary particularises Theorem 3 to the (almost) lossless reconstruction setting. We omit the proof.

Corollary 4. For Hamming distortion functions,

$$
\mathrm{R}^{\dagger}(\mathbf{0}, C)=\mathrm{R}^{\ddagger}(\mathbf{0}, C)=\mathrm{R}(\mathbf{0}, C)=\min _{U: I(\boldsymbol{X} ; U) \leq C} \max _{\ell \in \mathcal{L}} H\left(X_{\ell} \mid U\right),
$$

where $\mathcal{U}$ can be restricted to $|\mathcal{U}| \leq|\mathcal{X}|+L$. 


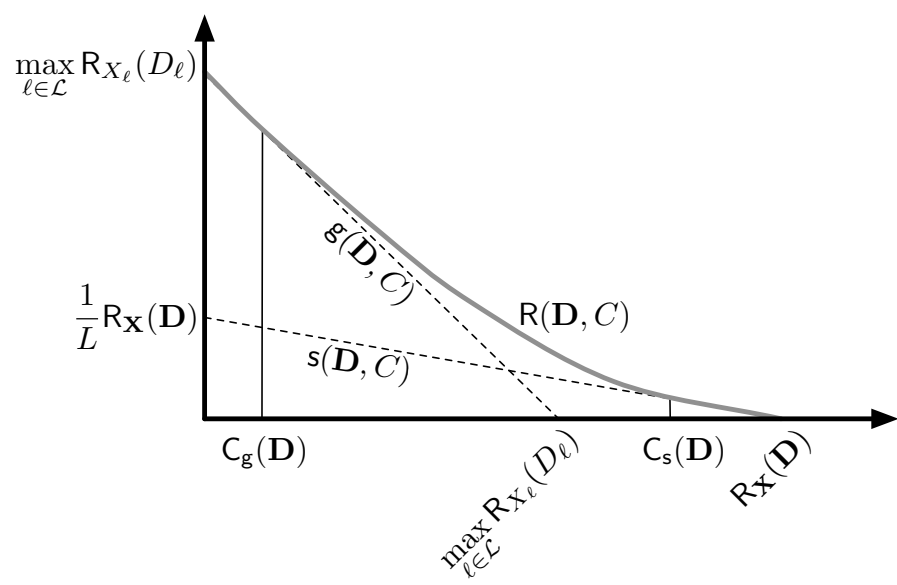

Fig. 2. An illustration of some typical characteristics of the RDC function $\mathrm{R}(\boldsymbol{D}, C)$ for a fixed distortion tuple $\boldsymbol{D}=\left(D_{1}, \ldots, D_{L}\right)$. The function describes the optimal (minimum) delivery-phase rate (vertical axis) for a given cache capacity (horizontal axis). The bounds in Lemmas 5 and 11 are plotted with dashed lines.

\section{CACHING INTERPRETATIONS OF THE GÁCS-KÖRNER AND WyNER’s COMMON INFORMATION}

We now consider two special 'enhanced' caching setups and derive their RDC functions. We then show that the conditions under which the RDC function $\mathrm{R}(\boldsymbol{D}, C)$ of our original caching problem coincides with the RDC functions of these enhanced setups relates to the different common-information definitions of Gács, Körner, and Wyner.

\section{A. Genie-Aided Caching and Gács-Körner Common Information}

Imagine that, before the caching phase, a genie tells the server which $\ell \in \mathcal{L}$ the user will choose in the future. The optimal caching strategy for this hypothetical genie-aided system is obvious: We should compress the $\ell$-th source $X_{\ell}^{n}$ using an optimal $\mathrm{RD}$ code, cache $n C$ bits of the code's output, and then send the remaining bits during the delivery phase. The RDC function of the genie-aided problem is therefore

$$
\mathrm{g}(\boldsymbol{D}, C)=\left[\max _{\ell \in \mathcal{L}} \mathrm{R}_{X_{\ell}}\left(D_{\ell}\right)-C\right]^{+}
$$

where $[a]^{+}=\max \{0, a\}$.

In the main problem at hand, however, the server does not know in advance which $\ell \in \mathcal{L}$ the user will select, and this uncertainty may cost additional rate in either the caching or delivery phases. Since the optimal performance of the genie-aided system cannot be worse than that of our system, we immediately have the following lemma.

Lemma 5. $\mathrm{R}(\boldsymbol{D}, C) \geq \mathrm{g}(\boldsymbol{D}, C)$.

Clearly, in the caching system, we can always achieve the genie bound at $C=0$. It is therefore natural to consider the critical cache capacity

$$
\mathrm{C}_{\mathrm{g}}(\boldsymbol{D}):=\max \{C \geq 0: \mathrm{R}(\boldsymbol{D}, C)=\mathrm{g}(\boldsymbol{D}, C)\} .
$$

That is, $\mathrm{C}_{\mathrm{g}}(\boldsymbol{D})$ is the largest cache capacity so that there is no loss in performance because the transmitter has to fill the cache memory before learning the demand $\ell$. The maximum indicated in $(8)$ exists because, for $0 \leq C \leq \mathrm{R}_{\boldsymbol{X}}(\boldsymbol{D}), \mathrm{R}(\boldsymbol{D}, C)$ is convex and $\mathrm{g}(\boldsymbol{D}, C)$ is linear. Figure 2 illustrates some typical characteristics of $\mathrm{R}(\boldsymbol{D}, C)$ and $\mathrm{g}(\boldsymbol{D}, C)$.

We now consider the critical cache capacity $\mathrm{C}_{\mathrm{g}}(\boldsymbol{D})$. Let

$$
\mathcal{L}^{*}(\boldsymbol{D}):=\left\{\ell^{*} \in \mathcal{L}: \mathrm{R}_{X_{\ell^{*}}}\left(D_{\ell^{*}}\right)=\max _{\ell \in \mathcal{L}} \mathrm{R}_{X_{\ell}}\left(D_{\ell}\right)\right\} .
$$

Define

$$
\mathrm{C}_{\mathrm{g}}^{*}(\boldsymbol{D}):=\max _{U} I(\boldsymbol{X} ; U),
$$

where the maximisation is taken over the set of all auxiliary random variables $U$ on $\mathcal{U}$ jointly distributed with $\boldsymbol{X}$ such that for all $\ell^{*} \in \mathcal{L}^{*}$ we have

$$
I(\boldsymbol{X} ; U)=\mathrm{R}_{X_{\ell^{*}}}\left(D_{\ell^{*}}\right)-\mathrm{R}_{X_{\ell^{*}} \mid U}\left(D_{\ell^{*}}\right)
$$

and

$$
\mathrm{R}_{X_{\ell^{*}} \mid U}\left(D_{\ell^{*}}\right)=\max _{\ell \in \mathcal{L}} \mathrm{R}_{X_{\ell} \mid U}\left(D_{\ell}\right) .
$$


Theorem 6. For separable distortion functions,

$$
\mathrm{C}_{\mathrm{g}}(\boldsymbol{D})=\mathrm{C}_{\mathrm{g}}^{*}(\boldsymbol{D})
$$

Corollary 7. For Hamming distortions

$$
\mathrm{C}_{\mathrm{g}}(\mathbf{0})=\mathrm{C}_{\mathrm{g}}^{*}(\mathbf{0})=\max _{U} I(\boldsymbol{X} ; U),
$$

where the maximisation is taken over the set of all $U$ satisfying

$$
U \leftrightarrow X_{\ell^{*}} \leftrightarrow X_{\mathcal{L} \backslash \ell^{*}} \quad \forall \ell^{*} \in \mathcal{L}^{*}
$$

and

$$
H\left(X_{\ell^{*}} \mid U\right)=\max _{\ell \in \mathcal{L}} H\left(X_{\ell} \mid U\right) \quad \forall \ell^{*} \in \mathcal{L}^{*} .
$$

Proof: Theorem 6 and Corollary 7 are proved in Appendices B-A and B-B respectively.

We now explain Corollary 7 at hand of an easy example. Suppose that we only have two sources $X_{1}=\left(A, B_{1}\right)$ and $X_{2}=\left(A, B_{2}\right)$ where $A, B_{1}$ and $B_{2}$ are mutually independent and $H\left(B_{1}\right)=H\left(B_{2}\right)$. In this case, choose $U=A$. This way, $\mathrm{C}_{\mathrm{g}}^{*}(\mathbf{0})=H(A)$ and

$$
\mathrm{R}(\mathbf{0}, C)=\mathrm{g}(\mathbf{0}, C)=H(A)+H\left(B_{1}\right)-C, \quad C \leq H(A) .
$$

In this example the optimal $U$ in Corollary 7 is "common" to both sources. In fact, if we had placed additional private information about, say, $X_{1}$ (i.e., information about $B_{1}$ ) in the cache, then this information would be wasted whenever the user downloads $X_{2}$.

The above idea naturally leads to a multivariate version of Viswanatham, Akyol and Rose's [11] definition of lossy GácsKörner common information. (See [12] for Gács and Körner's original treatment of common information.)

Definition 5. Define the lossy Gács-Körner common information of $\boldsymbol{X}$ w.r.t. $\mathbf{d}$ by

$$
\mathrm{K}_{G K}(\boldsymbol{X} ; \boldsymbol{D}):=\max _{(U, \hat{\boldsymbol{X}})} I(\boldsymbol{X} ; U),
$$

where the maximum is taken over all tuples $(U, \hat{\boldsymbol{X}})$ on $\mathcal{U} \times \hat{\boldsymbol{X}}$ jointly distributed with $\boldsymbol{X}$ and satisfying

(i) $\forall \ell \in \mathcal{L}: U \leftrightarrow X_{\ell} \leftrightarrow X_{\mathcal{L} \backslash \ell}$

(ii) $\forall \ell \in \mathcal{L}: U \leftrightarrow \hat{X}_{\ell} \leftrightarrow X_{\ell}$

(iii) $\forall \ell \in \mathcal{L}: \mathbb{E}\left[\mathrm{d}_{\ell}\left(\hat{X}_{\ell}, X_{\ell}\right)\right] \leq D_{\ell}$

(iv) $\forall \ell \in \mathcal{L}: I\left(X_{\ell} ; \hat{X}_{\ell}\right)=\mathrm{R}_{X_{\ell}}\left(D_{\ell}\right)$.

The indicated maximum in Definition 5 exists because the set of all tuples $(U, \hat{\boldsymbol{X}})$ satisfying (i)-(iv) can be viewed as a compact subset of the corresponding probability simplex.

The next theorem relates the critical cache capacity $\mathrm{C}_{\mathrm{g}}^{*}(\boldsymbol{D})$ to $\mathrm{K}_{\mathrm{GK}}(\boldsymbol{X} ; \boldsymbol{D})$, and it provides a new operational meaning for lossy Gács-Körner common information in caching systems.

Theorem 8. For separable distortion functions,

$$
\mathrm{C}_{\mathrm{g}}^{*}(\boldsymbol{D}) \geq \mathrm{K}_{G K}(\boldsymbol{X} ; \boldsymbol{D})
$$

with equality if $\mathrm{R}_{X_{1}}\left(D_{1}\right)=\cdots=\mathrm{R}_{X_{L}}\left(D_{L}\right)$.

Proof: Theorem 8 is proved in Appendix C

Now recall the multivariate extension of Gács and Körner's original (lossless) definition of common information in [12]:

Definition 6. Gács and Körner's common information of the tuple $\boldsymbol{X}$ is defined as

$$
\mathrm{K}_{G K}(\boldsymbol{X}):=\max _{U: U \leftrightarrow X_{\ell} \leftrightarrow X_{\mathcal{L} \backslash \ell}, \forall \ell \in \mathcal{L}} I(\boldsymbol{X} ; U),
$$

where the maximisation is taken over all auxiliary random variables $U$ on $\mathcal{U}$ jointly distributed with $\boldsymbol{X}$ satisfying the $L$ indicated Markov chains.

The definition of the lossy Gács-Körner common information $\mathrm{K}_{\mathrm{GK}}(\boldsymbol{X} ; \boldsymbol{D})$ in $[10$ and the definition of the original GácsKörner common information $\mathrm{K}_{\mathrm{GK}}(\boldsymbol{X})$ in (11) can apply both to discrete random vectors $\boldsymbol{X}$ as well as continuous random vectors $\boldsymbol{X}$. In this latter case, however, the lossy Gács-Körner common information is only defined when all rate-distortion functions in (iv) are finite,

$$
\mathrm{R}_{X_{\ell}}\left(D_{\ell}\right)<\infty, \forall \ell \in \mathcal{L} .
$$

This finiteness can be guaranteed by, for example, our assumption that the single symbol distortion function $d_{\ell}$ satisfies $d_{\ell}\left(\hat{x}_{\ell}, x_{\ell}\right)<D_{\max }$ for all $\hat{x}_{\ell}$ and $x_{\ell}$. 
The optimization variable $U$ is subject to less constraints in (11) than 10$]$, so

$$
\mathrm{K}_{\mathrm{GK}}(\boldsymbol{X} ; \boldsymbol{D}) \leq \mathrm{K}_{\mathrm{GK}}(\boldsymbol{X})
$$

whenever (12) holds. Moreover, for discrete random variables $\boldsymbol{X}$ and Hamming distortion functions $\mathbf{d}$ :

$$
\mathrm{K}_{\mathrm{GK}}(\boldsymbol{X} ; \boldsymbol{D}=\mathbf{0})=\mathrm{K}_{\mathrm{GK}}(\boldsymbol{X}) .
$$

Lemma 9. Let $\mathbf{d}$ be Hamming distortion functions and consider zero distortions, $\boldsymbol{D}=\mathbf{0}$. Then,

$$
\mathrm{K}_{G K}(\boldsymbol{X})=\max _{U: H\left(U \mid X_{\ell}\right)=0, \forall \ell \in \mathcal{L}} H(U)
$$

Proof: Lemma 9 is proved in Appendix $\mathrm{D}$.

This leads the following corollary:

Corollary 10. For Hamming distortion functions we have

$$
\mathrm{C}_{\mathrm{g}}^{*}(\mathbf{0}) \geq \mathrm{K}_{G K}(\boldsymbol{X})
$$

with equality if $H\left(X_{1}\right)=H\left(X_{2}\right)=\ldots=H\left(X_{L}\right)$.

\section{B. The Superuser Setup and Wyner's Common Information}

Now imagine that a superuser is connected to the server by $L$ independent rate $R$ noiseless links, and suppose that the superuser requests every source. The optimal caching strategy for this superuser problem is again clear: Take an optimal code for the joint RD function of $\boldsymbol{X}$, cache $C$ bits of the code's output, and distribute the remaining bits equally over the $L$ links in the delivery phase. The RDC function of this superuser problem is

$$
\mathrm{s}(\boldsymbol{D}, C)=\left[\frac{\mathrm{R}_{\boldsymbol{X}}(\boldsymbol{D})-C}{L}\right]^{+} .
$$

Returning to our main problem: The server only receives one source request and, therefore, it cannot distribute and average the compressed bits of the joint RD code over $L$ noiseless channels. This intuition leads to the following bound.

\section{Lemma 11.}

$$
\mathrm{R}(\boldsymbol{D}, C) \geq \mathrm{s}(\boldsymbol{D}, C) \text {. }
$$

Clearly the superuser bound is achievable by the caching system at $C=\mathrm{R}_{\boldsymbol{X}}(\boldsymbol{D})$ and, similarly to the previous subsection, it is natural to consider the smallest cache capacity for which there is no rate loss with respect to the optimal superuser system:

$$
\mathrm{C}_{\mathrm{s}}(\boldsymbol{D}):=\min \{C \geq 0: \mathrm{R}(\boldsymbol{D}, C)=\mathrm{s}(\boldsymbol{D}, C)\}
$$

The minimum in (14) exists because, for $0 \leq C \leq \mathrm{R}_{\boldsymbol{X}}(\boldsymbol{D}), \mathrm{R}_{\boldsymbol{X}}(\boldsymbol{D})$ is convex and $\mathrm{s}(\boldsymbol{D}, C)$ is linear. Figure 2 depicts the superuser bound and the critical cache capacity $\mathrm{C}_{\mathrm{s}}(\boldsymbol{D})$.

We now characterise $C_{s}(D)$. For a given $D$, let

$$
\mathrm{C}_{\mathrm{s}}^{*}(\boldsymbol{D}):=\min _{(U, \hat{\boldsymbol{X}})} I(\boldsymbol{X} ; U)
$$

where the minimum is taken over all tuples $(U, \hat{\boldsymbol{X}})$ on $\mathcal{U} \times \hat{\mathcal{X}}$ such that the following five properties hold

(i) $\boldsymbol{X} \leftrightarrow \hat{\boldsymbol{X}} \leftrightarrow U$

(ii) $I\left(X_{1} ; \hat{X}_{1} \mid U\right)=\cdots=I\left(X_{L} ; \hat{X}_{L} \mid U\right)$

(iii) $\forall \ell \in \mathcal{L}: \hat{X}_{\ell} \leftrightarrow U \leftrightarrow \hat{X}_{\mathcal{L} \backslash \ell}$

(iv) $\forall \ell \in \mathcal{L}: \mathbb{E}\left[\mathrm{d}_{\ell}\left(\hat{X}_{\ell}, X_{\ell}\right)\right] \leq D_{\ell}$

(v) $I(\boldsymbol{X} ; \hat{\boldsymbol{X}})=\mathrm{R}_{\boldsymbol{X}}(\boldsymbol{D})$.

Theorem 12. For separable distortion functions,

$$
\mathrm{C}_{\mathrm{s}}(\boldsymbol{D})=\mathrm{C}_{\mathrm{s}}^{*}(\boldsymbol{D})
$$

Proof: Theorem 12 is proved in Appendix E

The above quantity $\mathrm{C}_{\mathrm{s}}^{*}(\boldsymbol{D})$ is closely related to the natural multivariate extension of Viswanatha, Akyol and Rose's [11] informational definition of lossy Wyner common information.

Definition 7. For a given distortion tuple $\boldsymbol{D}$ and single-symbol distortion functions d, define the lossy Wyner common information of $\boldsymbol{X}$ by

$$
\mathrm{K}_{W}(\boldsymbol{X} ; \boldsymbol{D}):=\min _{(U, \hat{\boldsymbol{X}})} I(\boldsymbol{X} ; U)
$$


where the minimum is taken over all tuples $(U, \hat{\boldsymbol{X}})$ on $\mathcal{U} \times \hat{\mathcal{X}}$ such that the following four properties hold

(i) $\boldsymbol{X} \leftrightarrow \hat{\boldsymbol{X}} \leftrightarrow U$

(ii) $\forall \ell \in \mathcal{L}: \hat{X}_{\ell} \leftrightarrow U \leftrightarrow \hat{X}_{\mathcal{L} \backslash \ell}$

(iii) $\forall \ell \in \mathcal{L}: \mathbb{E}\left[\mathrm{d}_{\ell}\left(\hat{X}_{\ell}, X_{\ell}\right)\right] \leq D_{\ell}$

(iv) $I(\boldsymbol{X} ; \hat{\boldsymbol{X}})=\mathrm{R}_{\boldsymbol{X}}(\boldsymbol{D})$.

The next theorem follows trivially from the above definitions, and it gives an operational meaning for lossy Wyner common information for caching.

Theorem 13. For separable distortion functions,

$$
\mathrm{C}_{\mathrm{s}}^{*}(\boldsymbol{D}) \geq \mathrm{K}_{W}(\boldsymbol{X} ; \boldsymbol{D})
$$

Now recall the natural multivariate extension of Wyner's original definition of common information [13]:

Definition 8. Wyner's common information of the tuple $\boldsymbol{X}$ is defined as

$$
\mathrm{K}_{W}(\boldsymbol{X}):=\min _{U: X_{\ell} \leftrightarrow U \leftrightarrow X_{\mathcal{L} \backslash \ell}, \forall \ell \in \mathcal{L}} I(\boldsymbol{X} ; U),
$$

where the minimum is taken over all auxiliary random variables satisfying all L indicated Markov chains.

The lossy Wyner common information $\mathrm{K}_{\mathrm{W}}(\boldsymbol{X} ; \boldsymbol{D})$ as well as Wyner's original common information $\mathrm{K}_{\mathrm{W}}(\boldsymbol{X})$ are both defined for discrete and continuous random vectors $\boldsymbol{X}$. In the latter case, the lossy Wyner common information is only defined when the rate-distortion function in (iv) is finite, $\mathrm{R}_{\boldsymbol{X}}(\boldsymbol{D})<\infty$.

Remark 1. At this point it is worth noting that, in general, the lossy Wyner common information $\mathrm{K}_{W}(\boldsymbol{X} ; \boldsymbol{D})$ is neither convex/concave nor monotonic in $\boldsymbol{D}$. Moreover, it is generally the case that $\mathrm{K}_{W}(\boldsymbol{X} ; \boldsymbol{D})$ can be larger/smaller than the Wyner common information $\mathrm{K}_{W}(\boldsymbol{X})$. A nice treatment of this issue for $L=2$ variables is given by Viswanatha et al. in [11. Sec. III.B].

Let now $\boldsymbol{X}$ be a discrete random vector and $\mathbf{d}$ be Hamming distortion functions. Consider zero distortions, $\boldsymbol{D}=\mathbf{0}$. Then,

$$
\mathrm{K}_{\mathrm{W}}(\boldsymbol{X})=\mathrm{K}_{\mathrm{W}}(\boldsymbol{X} ; \boldsymbol{D}=\mathbf{0}),
$$

which implies the following corollary.

Corollary 14. For Hamming distortions we have that

$$
\mathrm{C}_{\mathrm{s}}^{*}(\mathbf{0}) \geq \mathrm{K}_{W}(\boldsymbol{X})
$$

with equality if $\mathrm{K}_{W}(\boldsymbol{X})=I\left(\boldsymbol{X} ; U^{*}\right)$ for some $U^{*}$ satisfying

- $H\left(X_{1} \mid U^{*}\right)=\cdots=H\left(X_{L} \mid U^{*}\right)$

- $\forall \ell \in \mathcal{L}: X_{\ell} \leftrightarrow U^{*} \leftrightarrow U_{\mathcal{L} \backslash \ell}^{*}$

\section{The Super-Genie Lower Bound}

We conclude this section by combining and generalising the genie and superuser lower bounds. For each subset $\mathcal{S} \subseteq \mathcal{L}$, let

$$
D_{\mathcal{S}}=\left(D_{\ell} ; \ell \in \mathcal{S}\right) \quad \text { and } \quad X_{\mathcal{S}}=\left(X_{\ell} ; \ell \in \mathcal{S}\right)
$$

and let

$$
\mathrm{R}_{X_{\mathcal{S}}}\left(D_{\mathcal{S}}\right)=\min _{p_{\hat{X}_{\mathcal{S}} \mid X_{S}}} I\left(X_{\mathcal{S}} ; \hat{X}_{\mathcal{S}}\right)
$$

denote the joint RD function of $X_{\mathcal{S}}$, where the minimum is taken over all test channels $p_{\hat{X}_{\mathcal{S}} \mid X_{S}}$ from $\mathcal{X}_{\mathcal{S}}$ to $\hat{\mathcal{X}}_{\mathcal{S}}$ such that $\mathbb{E}\left[\mathrm{d}_{\ell}\left(\hat{X}_{\ell}, X_{\ell}\right)\right] \leq D_{\ell}$ for all $\ell \in \mathcal{S}$.

\section{Lemma 15.}

$$
\mathrm{R}(\boldsymbol{D}, C) \geq \max _{\mathcal{S} \subseteq \mathcal{L}}\left[\frac{\mathrm{R}_{X_{\mathcal{S}}}\left(D_{\mathcal{S}}\right)-C}{|\mathcal{S}|}\right]^{+}
$$

Proof: Lemma 15 is proved in Appendix F 


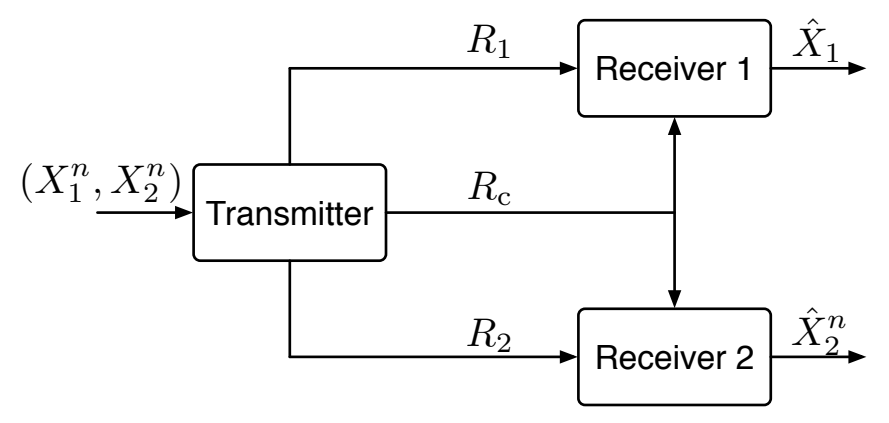

Fig. 3. Lossy source coding for a simple network with two receivers.

\section{CONNECTIONS TO GRAY AND WYNER's “SOURCE CODING FOR A SIMPLE NETWORK”}

The RDC function in (77) is closely related to Gray and Wyner's classic "source coding for a simple network" problem [14] illustrated in Figure 3. A transmitter is connected to two different receivers via a common link of rate $R_{\mathrm{c}}$ and two private links of rates $R_{1}$ and $R_{2}$. The set of all achievable rate tuples $\left(R_{\mathrm{c}}, R_{1}, R_{2}\right)$ for which receivers 1 and 2 can respectively reconstruct $X_{1}^{n}$ and $X_{2}^{n}$ to within distortions $D_{1}$ and $D_{2}$ is given by [14. Thm. 8]

$$
\mathcal{R}_{\mathrm{GW}}\left(D_{1}, D_{2}\right):=\bigcup_{p_{U \mid X_{1}, X_{2}}}\left\{\left(R_{\mathrm{c}}, R_{1}, R_{2}\right): \quad \begin{array}{l}
R_{\mathrm{c}} \geq I\left(X_{1}, X_{2} ; U\right) \\
R_{1} \geq \mathrm{R}_{X_{1} \mid U}\left(D_{1}\right) \\
R_{2} \geq \mathrm{R}_{X_{2} \mid U}\left(D_{2}\right)
\end{array}\right\},
$$

where the union is over all test channels $p_{U \mid X_{1}, X_{2}}$ from $\mathcal{X}_{1} \times \mathcal{X}_{2}$ to an auxiliary alphabet $\mathcal{U}$ of cardinality $|\mathcal{U}| \leq\left|\mathcal{X}_{1}\right|\left|\mathcal{X}_{2}\right|+4$.

It is possible to extend Gray and Wyner's "simple network" from $L=2$ receivers to an arbitrary number of receivers. More specifically suppose that the transmitter is connected to $L$ different receivers via a single common link with rate $R_{\mathrm{c}}$ and $L$ private links of rates $\boldsymbol{R}=\left(R_{1}, R_{2}, \ldots, R_{L}\right)$ respectively, where $R_{\ell}$ denotes the rate to the $\ell$-th receiver. It is not too hard to show that the set of all achievable rate tuples $\left(R_{\mathrm{c}}, \boldsymbol{R}\right)$ for which every receiver $\ell$ can reconstruct $X_{\ell}^{n}$ to within a distortion $D_{\ell}$ is given by

$$
\mathcal{R}_{\mathrm{GW}}(\boldsymbol{D}):=\bigcup_{p_{U \mid X}}\left\{\left(R_{\mathrm{c}}, \boldsymbol{R}\right): \begin{array}{l}
R_{\mathrm{c}} \geq I(\boldsymbol{X} ; U) \\
R_{\ell} \geq \mathrm{R}_{X_{\ell} \mid U}\left(D_{\ell}\right) \quad \forall \ell \in \mathcal{L} .
\end{array}\right\},
$$

where the union is over all test channels $p_{U \mid X_{1}, X_{2}}$ from $\mathcal{X}$ to $\mathcal{U}$ with $|\mathcal{U}| \leq|\mathcal{X}|+2 L$. The next proposition shows that the informational RDC function can be expressed as a minimisation over the achievable rate region $\mathcal{R}_{\mathrm{GW}}(\boldsymbol{D})$.

Lemma 16.

$$
\mathrm{R}(\boldsymbol{D}, C)=\min _{(C, \boldsymbol{R}) \in \mathcal{R}_{G W}(\boldsymbol{D})} \max _{\ell \in \mathcal{L}} R_{\ell} .
$$

Proof: If $(C, \boldsymbol{R}) \in \mathcal{R}_{\mathrm{GW}}(\boldsymbol{D})$, then we can use the corresponding Gray-Wyner encoder and decoders to achieve a delivery phase-rate of $\max _{\ell} R_{\ell}$ in the caching problem; thus, $\mathrm{R}(\boldsymbol{D}, C)$ cannot be larger than the minimum in Lemma 16 . Now suppose $\mathrm{R}(\boldsymbol{D}, C)$ is strictly smaller than the above minimum: There would then exist an encoder and decoders in the Gray-Wyner problem that can operate outside of the rate region $\mathcal{R}_{\mathrm{GW}}(\boldsymbol{D})$, which is a contradiction.

\section{Vi. A Strong Converse For Separable Distortion Functions}

Consider the excess distortion problem formulation in Definition 4 for the special case of separable distortion functions $\overline{\mathbf{d}}$ in (2). If the delivery-phase rate $R$ is strictly smaller than the informational $\operatorname{RDC}$ function $\mathrm{R}(\boldsymbol{D}, C)$, then the weak converse part of Theorem 3 shows that the excess-distortion probability of any sequence of $\left(n, \mathcal{M}_{\mathrm{c}}^{(n)}, \mathcal{M}^{(n)}\right)$ codes satisfying 5 a and (5b) will be bounded away from zero; that is,

$$
\limsup _{n \rightarrow \infty} \mathbb{P}\left[\bigcup_{\ell \in \mathcal{L}}\left\{\overline{\mathrm{d}}_{\ell}\left(\hat{X}_{\ell}^{n}, X_{\ell}^{n}\right) \geq D_{\ell}\right\}\right]>0 .
$$

The next theorem strengthens this weak converse, and it will be used for $\mathrm{f}$-separable distortion functions in the next section.

Theorem 17. Fix any cache capacity $C$ and distortion tuple $\boldsymbol{D}$ such that $\mathrm{R}(\boldsymbol{D}, C)>0$. Any sequence of $\left(n, \mathcal{M}_{c}^{(n)}, \mathcal{M}^{(n)}\right)$-codes satisfying

$$
\limsup _{n \rightarrow \infty} \frac{1}{n} \log \left|\mathcal{M}^{(n)}\right|<\mathrm{R}(\boldsymbol{D}, C)
$$


and

$$
\limsup _{n \rightarrow \infty} \frac{1}{n} \log \left|\mathcal{M}_{c}^{(n)}\right| \leq C
$$

must also satisfy

$$
\limsup _{n \rightarrow \infty} \mathbb{P}\left[\bigcup_{\ell \in \mathcal{L}}\left\{\overline{\mathrm{d}}_{\ell}\left(\hat{X}_{\ell}^{n}, X_{\ell}^{n}\right) \geq D_{\ell}\right\}\right]=1 .
$$

Proof: Theorem 17 is proved in Appendix $\mathrm{G}$

The strong converse in Theorem 17 applies to the union of excess-distortion events in (18). As an alternative to this union event, one could also consider the maximum excess distortion probability across source files. The following lemmas 18 and 19 present results on maximum excess distortion probabilities. The next lemma follows by modifying the strong converse for the usual point-to-point RD problem (see, for example, Kieffer [15]). We omit its proof.

Lemma 18. Fix any cache capacity $C$ and distortion tuple $\boldsymbol{D}$ such that $\mathrm{R}(\boldsymbol{D}, C)>0$. Any sequence of $\left(n, \mathcal{M}_{c}^{(n)}, \mathcal{M}^{(n)}\right)$-codes satisfying (17) and

$$
\limsup _{n \rightarrow \infty} \frac{1}{n} \log \left|\mathcal{M}^{(n)}\right|+C<\mathrm{R}_{X_{\ell}}\left(D_{\ell}\right)
$$

for some $\ell \in \mathcal{L}$ must also satisfy

$$
\limsup _{n \rightarrow \infty} \mathbb{P}\left[\overline{\mathrm{d}}_{\ell}\left(\hat{X}_{\ell}^{n}, X_{\ell}^{n}\right) \geq D_{\ell}\right]=1 .
$$

Lemma 18 and the definition of the critical cache capacity $C_{g}(D)$ in $(8)$ immediately yield the following marginal strong converse for small cache sizes. We omit its proof.

Lemma 19. Fix any cache capacity $C$ and distortion tuple $\boldsymbol{D}$ such that $\mathrm{R}(\boldsymbol{D}, C)>0$ and $C \leq \mathrm{C}_{\mathrm{g}}(\boldsymbol{D})$. Any sequence of $\left(n, \mathcal{M}_{c}^{(n)}, \mathcal{M}^{(n)}\right)$-codes satisfying 17 and

$$
\limsup _{n \rightarrow \infty} \frac{1}{n} \log \left|\mathcal{M}^{(n)}\right|<\mathrm{R}(\boldsymbol{D}, C)
$$

must also satisfy (19) for some $\ell \in \mathcal{L}$.

\section{ExAmples (SEPARABle Distortion Functions)}

\section{A. Identical and Independent Sources}

Suppose that $\boldsymbol{X}=\left(X_{1}, \ldots, X_{L}\right)$ is a string of $L$ mutually independent instances of a random variable $X$ on $\mathcal{X}$. If $\mathrm{d}_{1}=\cdots=\mathrm{d}_{L}=\mathrm{d}$ and $\boldsymbol{D}=(D, \ldots, D)$, then Theorem 3 specializes to:

$$
\mathrm{R}(\boldsymbol{D}, C)=\mathrm{s}(\boldsymbol{D}, C)=\left[\mathrm{R}_{X}(D)-\frac{C}{L}\right]^{+} .
$$

The optimal caching strategy here is simple: Take an optimal RD code for $(X, \overline{\mathrm{d}}, D)$; compress each source $X_{\ell}^{n}$ to the RD limit $\mathrm{R}_{X}(D)$; cache $C / L$ of the compressed bits; and transmit the remaining bits during the delivery phase.

\section{B. Multivariate Gaussian Sources}

The discussion so far has been restricted to sources defined on finite alphabets. However, it can be shown that Theorem 3 extends to multivariate Gaussian sources with squared-error distortions; for example, see the discussion in [16].

More formally, let $\boldsymbol{X}=\left(X_{1}, \ldots, X_{L}\right) \in \mathbb{R}^{L}$ be a zero mean multivariate Gaussian with covariance matrix $\boldsymbol{K}_{\boldsymbol{X}}$ and

$$
\mathrm{d}_{\ell}\left(\hat{x}_{\ell}, x_{\ell}\right)=\left(\hat{x}_{\ell}-x_{\ell}\right)^{2}, \quad \forall \ell \in \mathcal{L} \text {. }
$$

Let $\mathrm{R}_{\mathrm{G}}^{\dagger}(\boldsymbol{D}, C)$ denote the corresponding operational RDC function w.r.t. the expected distortion criteria:

$$
\mathbb{E}\left[\frac{1}{n} \sum_{i=1}^{n}\left(\hat{X}_{\ell, i}-X_{\ell, i}\right)^{2}\right] \leq D_{i}, \quad \forall \ell \in \mathcal{L} .
$$

Now let

$$
\mathrm{R}_{\mathrm{G}}(\boldsymbol{D}, C)=\inf _{(U, \hat{\boldsymbol{X}})} \max _{\ell \in \mathcal{L}} I\left(X_{\ell} ; \hat{X}_{\ell} \mid U\right)
$$

where the infinum is taken over all real tuples $(U, \hat{\boldsymbol{X}})$ jointly distributed with $\boldsymbol{X}$ such that

$$
I(\boldsymbol{X} ; U) \leq C
$$

and

$$
\mathbb{E}\left[\left(X_{\ell}-\hat{X}_{\ell}\right)^{2}\right] \leq D_{\ell}, \quad \forall \ell \in \mathcal{L}
$$




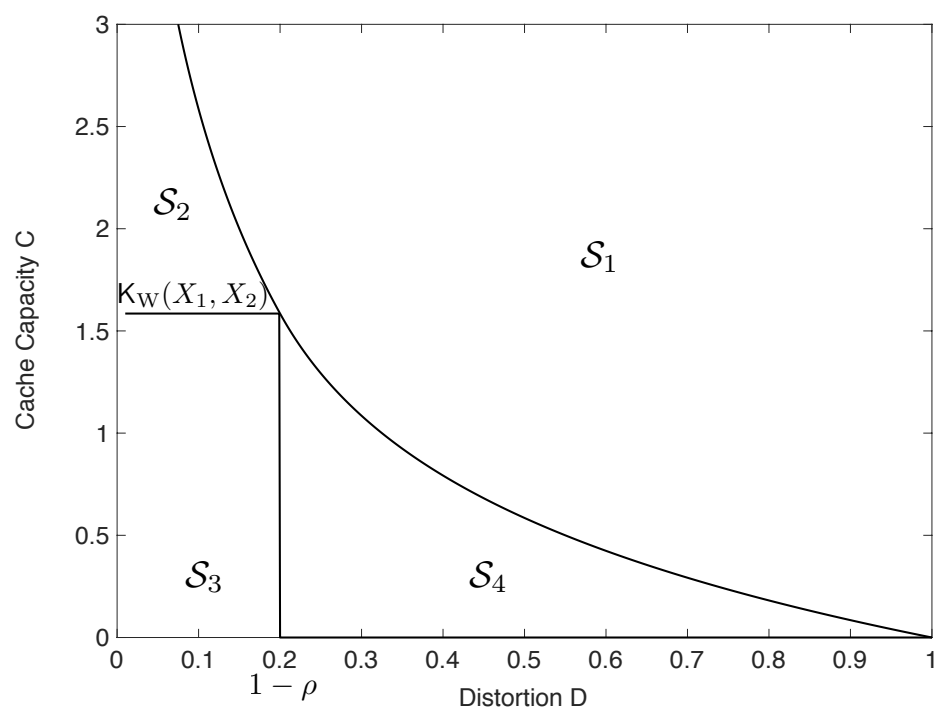

Fig. 4. Illustration of the distortion-cache regions $\mathcal{S}_{1}, \mathcal{S}_{2}, \mathcal{S}_{3}$ and $\mathcal{S}_{4}$ used in Theorem 22 with $\rho=0.8$.

The next theorem is the Gaussian counterpart of Theorem 3, and we omit its proof.

\section{Theorem 20.}

$$
\mathrm{R}_{G}(\boldsymbol{D}, C)=\mathrm{R}_{G}^{\dagger}(\boldsymbol{D}, C) .
$$

Strictly speaking, the expression we give in 20 for the Gaussian RDC function is non-computable, because there is no bound on the cardinality on the auxiliary variable $U$ (and it is not clear if one can restrict the optimization domain to $U$ 's that are jointly Gaussian with $\boldsymbol{X}$ ). As we will see in the next subsection, we can give explicit expressions for $\operatorname{R}_{\mathrm{G}}^{\dagger}(\boldsymbol{D}, C)$ when there are only two sources and over wide ranges of parameters of the Gaussian sources.

For an arbitrary number of source files and for symmetric distortions, we have the following lower bound on $\mathrm{R}_{\mathrm{G}}^{\dagger}(\boldsymbol{D}, C)$ in the next proposition. It is a Gaussian version of Lemma 15

For each subset $\mathcal{S} \subseteq \mathcal{L}$, let $X_{\mathcal{S}}=\left(X_{\ell} ; \ell \in \mathcal{S}\right)$ denote the tuple of random variables with indices in $\mathcal{S}$, and let $\boldsymbol{K}_{X_{\mathcal{S}}}$ denote the covariance matrix of $X_{\mathcal{S}}$.

Proposition 21. If $\boldsymbol{D}=(D, \ldots, D)$, then

$$
\mathrm{R}_{G}(\boldsymbol{D}, C) \geq \max _{\mathcal{S} \subseteq \mathcal{L}}\left[\frac{1}{2|\mathcal{S}|} \log \frac{\operatorname{det} \boldsymbol{K}_{X_{\mathcal{S}}}}{D^{|\mathcal{S}|}}-\frac{C}{|\mathcal{S}|}\right] .
$$

Proof: Proposition 21 is proved in Appendix 『

\section{Bivariate Gaussian Sources}

Let us now fix $\rho \in(0,1)$ and consider a zero mean bivariate Gaussian source $\boldsymbol{X}=\left(X_{1}, X_{2}\right)$ with the covariance matrix

$$
\boldsymbol{K}_{X_{1} X_{2}}=\left[\begin{array}{ll}
1 & \rho \\
\rho & 1
\end{array}\right] .
$$

We wish to evaluate the Gaussian RDC function in (20) with symmetric distortions $D_{1}=D_{2}=D$. To do this, we will consider distortion-cache pairs $(D, C)$ separately for each one of the regions $\mathcal{S}_{1}, \mathcal{S}_{2}, \mathcal{S}_{3}$ and $\mathcal{S}_{4}$ that we define shortly. There are two key quantities defining these regions: the Gaussian joint RD function $\mathrm{R}_{\mathrm{G}, X_{1} X_{2}}$ and the Wyner common information between $X_{1}$ and $X_{2}$. For symmetriq ${ }^{3}$ distortions $D_{1}=D_{2}=D$, the joint RD function $\mathrm{R}_{\mathrm{G}, X_{1} X_{2}}$ is given by [18. Thm. III.1]:

(i) If $0<D \leq 1-\rho$, then

$$
\mathrm{R}_{\mathrm{G}, X_{1} X_{2}}(D, D)=\frac{1}{2} \log \frac{1-\rho^{2}}{D^{2}} .
$$

(ii) If $1-\rho \leq D \leq 1$, then

$$
\mathrm{R}_{\mathrm{G}, X_{1} X_{2}}(D, D)=\frac{1}{2} \log \frac{1+\rho}{2 D-(1-\rho)} .
$$

\footnotetext{
${ }^{3}$ Here we only recall the joint RD function of $\left(X_{1}, X_{2}\right)$ for the case of symmetric distortions, $D_{1}=D_{2}=D$. A treatment of the RD function for arbitrary distortion pairs can be found in [18] and the references therein.
} 
(iii) If $D>1$, then

$$
\mathrm{R}_{\mathrm{G}, X_{1} X_{2}}(D, D)=0
$$

The Wyner common information of the Gaussian pair $X_{1}$ and $X_{2}$ is given by [11, 17]

$$
\mathrm{K}_{\mathrm{W}}\left(X_{1}, X_{2}\right)=\frac{1}{2} \log \frac{1+\rho}{1-\rho}
$$

Notice that for this symmetric Gaussian example, the original Wyner common information $\mathrm{K}_{\mathrm{W}}\left(X_{1}, X_{2}\right)$ equals the lossy Wyner common information $\mathrm{K}_{\mathrm{W}}\left(X_{1}, X_{2} ; D, D\right)$ when $0<D<(1-\rho)$ [11, Eq. (30)].

We can now define the four regions $\mathcal{S}_{1}, \mathcal{S}_{2}, \mathcal{S}_{3}, \mathcal{S}_{4}$ :

$$
\begin{gathered}
\mathcal{S}_{1}:=\left\{(D, C): C \geq \mathrm{R}_{\mathrm{G}, X_{1} X_{2}}(D, D)\right\} \\
\mathcal{S}_{2}:=\left\{(D, C): \mathrm{K}_{\mathrm{W}}\left(X_{1}, X_{2}\right) \leq C \leq \mathrm{R}_{\mathrm{G}, X_{1} X_{2}}(D, D)\right\}, \\
\mathcal{S}_{3}:=\left\{(D, C): D \leq 1-\rho, C \leq \mathrm{K}_{\mathrm{W}}\left(X_{1}, X_{2}\right)\right\}
\end{gathered}
$$

and

$$
\mathcal{S}_{4}:=\left\{(D, C): 1-\rho \leq D \leq 1, C \leq \mathrm{R}_{\mathrm{G}, X_{1} X_{2}}(D, D)\right\} .
$$

The four regions are illustrated in Figure 4

Theorem 22. For the zero mean bivariate Gaussian source $\left(X_{1}, X_{2}\right)$ with the covariance matrix $\boldsymbol{K}_{X_{1} X_{2}}$ in 22 and squared error distortion constraints, we have

$$
\mathrm{R}_{G}((D, D), C)= \begin{cases}0, & (C, D) \in \mathcal{S}_{1}, \\ \frac{1}{4} \log \frac{1-\rho^{2}}{D^{2}}-\frac{C}{2}, & (C, D) \in \mathcal{S}_{2},\end{cases}
$$

and

$$
\mathrm{R}_{G}((D, D), C) \leq \frac{1}{2} \log \frac{1-\frac{1}{2}(1+\rho)\left(1-2^{-2 C}\right)}{D}, \quad(C, D) \in \mathcal{S}_{3} \cup \mathcal{S}_{4} .
$$

Proof: Theorem 22 is proved in Appendix $\mathrm{K}$

Figure 5 illustrates the results in this Theorem 22 and the previous Proposition 21 at hand of an example.

\section{Doubly Symmetric Binary Source}

We now evaluate the RDC function for a doubly symmetric binary source (DSBS) under Hamming distortion functions. Fix $0 \leq \rho \leq 1 / 2$. Suppose that the library consists of two sources, $\mathcal{X}_{1}=\mathcal{X}_{2}=\hat{\mathcal{X}}_{1}=\hat{\mathcal{X}}_{2}=\{0,1\}$ and

$$
p_{\boldsymbol{X}}\left(x_{1}, x_{2}\right)=\frac{1}{2}(1-\rho) \mathbb{1}\left\{x_{1}=x_{2}\right\}+\frac{1}{2} \rho \mathbb{1}\left\{x_{1} \neq x_{2}\right\} \text {. }
$$

Let

$$
\rho^{*}=\frac{1}{2}-\frac{1}{2} \sqrt{1-2 \rho}
$$

and note that Wyner's common information reduces to

$$
\begin{aligned}
\mathrm{K}_{\mathrm{W}}\left(X_{1}, X_{2}\right) & =\min _{U: X_{1} \leftrightarrow U \leftrightarrow X_{2}} I\left(X_{1}, X_{2} ; U\right) \\
& =1+h(\rho)-2 h\left(\rho^{*}\right) .
\end{aligned}
$$

Theorem 23. The following is true for the DSBS:

(i) $\mathrm{C}_{\mathrm{s}}^{*}(\mathbf{0})=\mathrm{K}_{W}\left(X_{1}, X_{2}\right)$ and

$$
\mathrm{R}(\mathbf{0}, C)=\frac{1+h(\rho)-C}{2}
$$

for all cache capacities $\mathrm{K}_{W}\left(X_{1}, X_{2}\right) \leq C \leq 1+h(\rho)$.

(ii) $\mathrm{R}(\mathbf{0}, 0)=1, \mathrm{C}_{\mathrm{g}}(\mathbf{0})=0$ and

$$
\mathrm{R}(\mathbf{0}, C)>[1-C]^{+}
$$




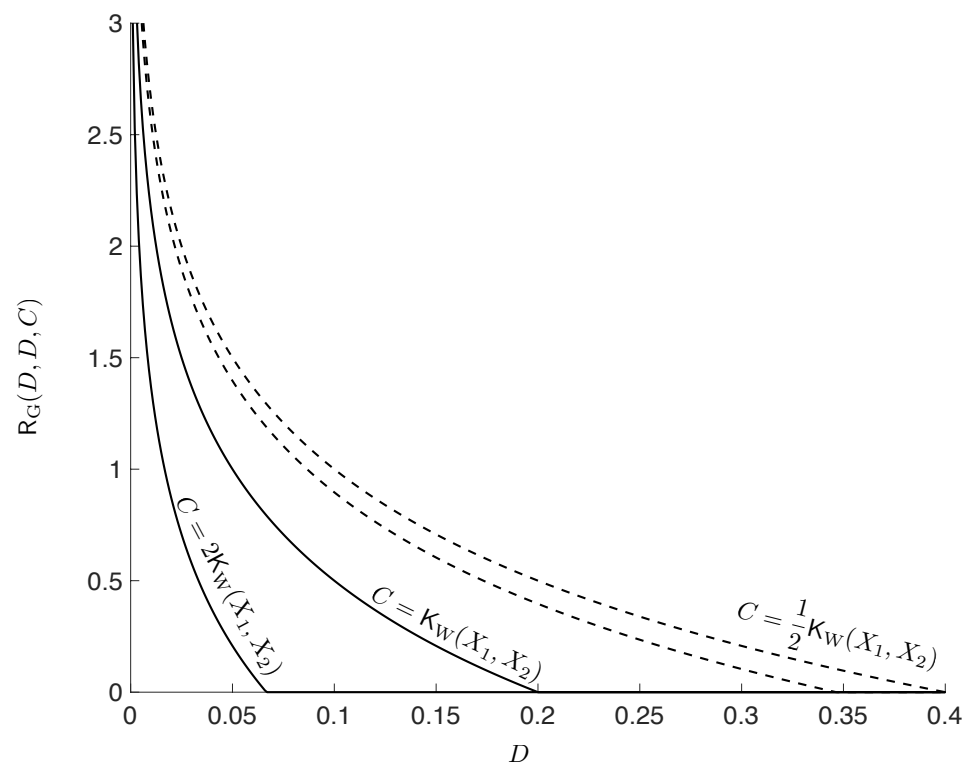

Fig. 5. Illustration of the RDC functions in Theorem 22 for a zero mean bivariate Gaussian source $\left(X_{1}, X_{2}\right)$ with the covariance matrix $\boldsymbol{K}_{X_{1} X_{2}}$ in 22 $(\rho=0.8)$, and symmetric distortion constraints $D_{1}=D_{2}=D$. The $\operatorname{RDC}$ function $\mathrm{R}_{\mathrm{G}}(D, D, C)$ is plotted as a function of the distortion $D$ for three difference cache capacities $C=2 \mathrm{~K}_{\mathrm{W}}\left(X_{1}, X_{2}\right), \mathrm{K}_{\mathrm{W}}\left(X_{1}, X_{2}\right)$ and $\left.(1 / 2) \mathrm{K}_{\mathrm{W}}\left(X_{1}, X_{2}\right)\right)$, where $\mathrm{K}_{\mathrm{W}}\left(X_{1}, X_{2}\right)$ denotes the Wyner common information 23 . For $C=(1 / 2) \mathrm{K}_{\mathrm{W}}\left(X_{1}, X_{2}\right)$, Proposition 21 and Theorem 22 only give lower and upper bounds, and these are shown with dashed lines.

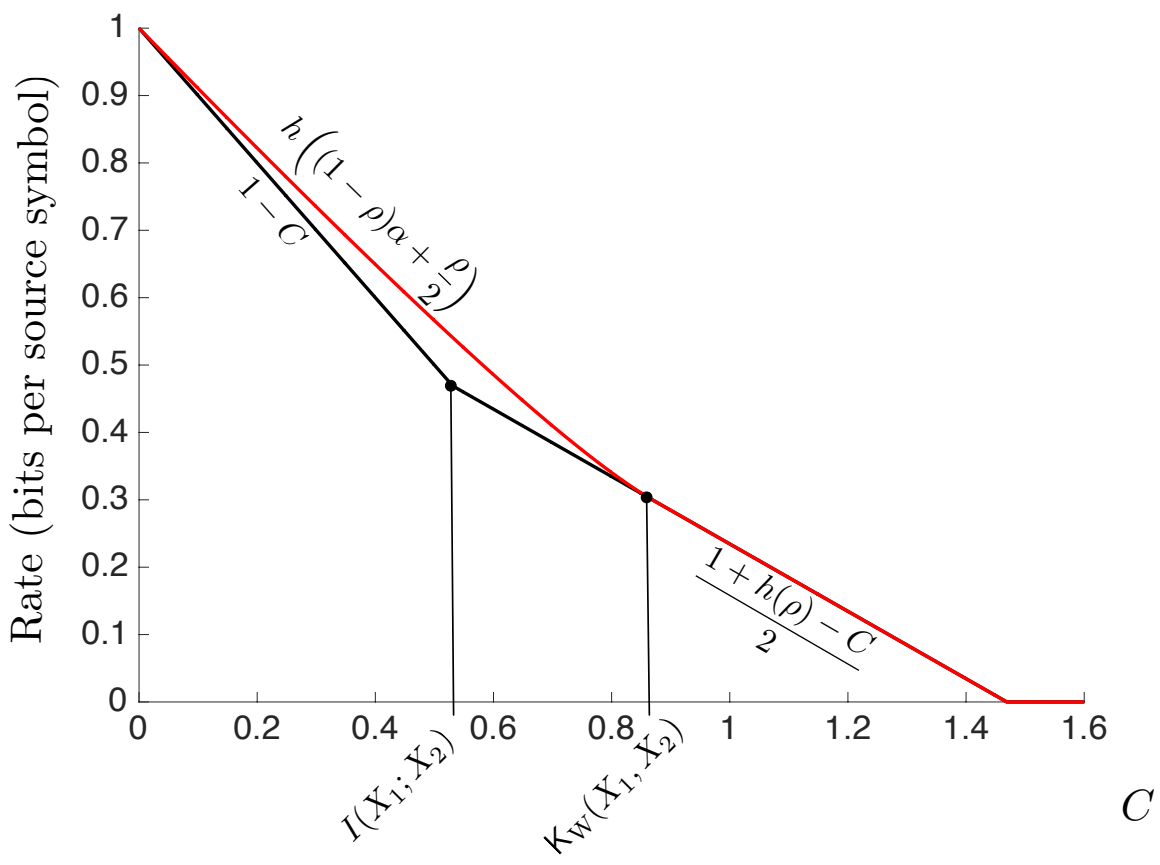

Fig. 6. Illustration of the upper (achievable) and lower (converse) bounds in Theorem 23 for the DSBS RDC function $\mathrm{R}(\mathbf{0}, C)$ with $\rho=0.1$.

for all cache capacities $0<C<1+h(\rho)$.

(iii)

$$
\frac{1+h(\rho)-C}{2} \leq \mathrm{R}(\mathbf{0}, C) \leq h\left((1-\rho) \alpha+\frac{\rho}{2}\right)
$$


for all cache capacities $0<C \leq \mathrm{K}_{W}\left(X_{1}, X_{2}\right)$, where

$$
\alpha=h^{-1}\left(\frac{1-\rho-C}{1-\rho}\right) .
$$

The above bounds are illustrated in Figure 6.

Proof: The fact that $\mathrm{R}(\mathbf{0}, C)$ is strictly greater than $[1-C]^{+}$for all positive $0<C<1+h(\rho)$ follows from Theorem 6 . Corollary 7, and $\mathrm{C}_{\mathrm{g}}^{*}(\mathbf{0})=\mathrm{K}_{\mathrm{GK}}(\boldsymbol{X} ; \mathbf{0})=0$. The remaining assertions in Theorem 23 can be proved using similar methods to evaluate the RDC function in Theorem 20 as in [14, Sec. 1.5], [19, Sec. III.C] and 17, Ex. 1], and we omit the details.

It is worth pointing out that, in this special case, the RDC function $\mathrm{R}(\boldsymbol{D}, C)$ particularises to the same expression as in [7. Ex. 1] (see also [14, Sec. 1.5]). Of course, this does not mean that Theorem 23 is a special case of the results in [7]; rather, the equivalence appears to be a consequence of the symmetry of the problem.

\section{THE RDC FUnCTION FOR f-SEPARABLE Distortion FunCtions}

Consider the operational RDC functions in Definitions 3 and 4 for the general case of $\mathrm{f}$-separable distortion functions (4), with $\mathbf{f}$ denoting the $L$ continuous and increasing functions in $(3)$ and $\mathbf{d}$ denoting the $L$ single-symbol distortion functions in (11).

For each request $\ell \in \mathcal{L}$ let

$$
\mathrm{d}_{\ell}^{*}: \hat{\mathcal{X}}_{\ell} \times \mathcal{X}_{\ell} \rightarrow[0, \infty)
$$

be the single-symbol distortion function obtained by setting

$$
\mathrm{d}_{\ell}^{*}\left(\hat{x}_{\ell}, x_{\ell}\right)=\mathrm{f}_{\ell}\left(\mathrm{d}_{\ell}\left(\hat{x}_{\ell}, x_{\ell}\right)\right) .
$$

Now let $\mathrm{R}_{\mathbf{d}^{*}}(\mathbf{f}(\boldsymbol{D}), C)$ denote the informational RDC function in (7) evaluated w.r.t. the single-symbol distortion functions

$$
\mathbf{d}^{*}=\left(\mathrm{d}_{1}^{*}, \ldots, \mathrm{d}_{L}^{*}\right)
$$

and distortion tuple

$$
\mathbf{f}(\boldsymbol{D})=\left(\mathrm{f}_{1}\left(D_{1}\right), \ldots, \mathrm{f}_{L}\left(D_{L}\right)\right) .
$$

Using Lemma 19 and the ideas in [3], it is not too difficult to obtain the following proposition. We omit the proof.

Proposition 24. For $\mathrm{f}$-separable distortion functions and all cache capacities $C \leq \mathrm{C}_{\mathrm{g}}(\boldsymbol{D})$, we have

$$
\mathbf{R}_{\mathbf{f}}^{\dagger}(\boldsymbol{D}, C)=\mathbf{R}_{\mathbf{f}}^{\ddagger}(\boldsymbol{D}, C)=\mathbf{R}_{\mathbf{d}^{*}}(\mathbf{f}(\boldsymbol{D}), C) .
$$

Proposition 24 is quite intuitive, and a natural question is whether or not it extends to cache capacities larger than $C_{g}(D)$. The next result considers such cases, but it requires a slightly more restricted version of the expected distortions operational model. Specifically, let us consider the following definition in place of Definition 3

Definition 9. We say that a rate-distortion-cache tuple $(R, \boldsymbol{D}, C)$ is achievable w.r.t. the expected max-distortion criterium if there exists a sequence of $\left(n, \mathcal{M}_{c}^{(n)}, \mathcal{M}^{(n)}\right)$-codes such that (5a) and (5b) hold and

$$
\limsup _{n \rightarrow \infty} \mathbb{E}\left[\max _{\ell \in \mathcal{L}}\left(\overline{\mathrm{fd}}_{\ell}\left(\hat{X}_{\ell}^{n}, X_{\ell}^{n}\right)-D_{\ell}\right)\right] \leq 0 .
$$

The RDC function w.r.t. expected max-distortions criterion is

$$
\tilde{\mathrm{R}}_{\mathbf{f}, \text { max-exc }}^{\dagger}(\boldsymbol{D}, C):=\inf \{R \geq 0:(R, \boldsymbol{D}, C) \text { is achievable w.r.t. expected max-distortions }\}
$$

Definition 9 is more restrictive than Definition 3 in the following sense: Any tuple $(R, D, C)$ that is achievable w.r.t. the expected max-distortion criteria is also achievable w.r.t. the expected distortion criteria. Therefore,

$$
\mathrm{R}_{\mathbf{f}}^{\dagger}(\boldsymbol{D}, C) \leq \tilde{\mathrm{R}}_{\mathbf{f}, \max -\mathrm{exc}}^{\dagger}(\boldsymbol{D}, C) .
$$

Theorem 25. For f-separable distortions we have

$$
\tilde{\mathrm{R}}_{\mathbf{f}, \text { max-exc }}^{\dagger}(\boldsymbol{D}, C)=\mathrm{R}_{\mathbf{f}}^{\ddagger}(\boldsymbol{D}, C)=\mathrm{R}_{\mathbf{d}^{*}}(\mathbf{f}(\boldsymbol{D}), C) .
$$

Proof: Theorem 25 is proved in Appendix [

\section{iX. Optimal Caching for Two Users (Separable Distortion Functions)}

This section generalises some of the ideas in previous sections from one user to two users. In particular, we will assume that user 1 has a cache with capacity $C$ while user 2 does not have a cache as illustrated Figure 7 


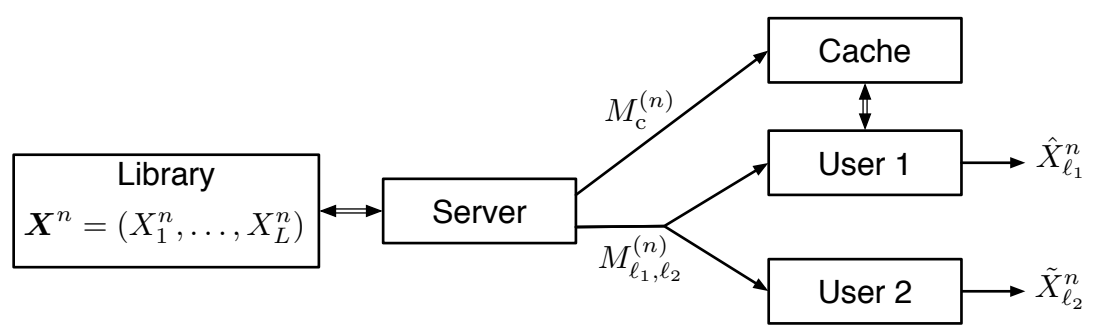

Fig. 7. Two-user RD cache problem.

\section{A. Setup}

Suppose that user 1 selects a source index $\ell_{1}$ from the set $\mathcal{L}_{1}$ and user 2 selects an index $\ell_{2}$ from the set $\mathcal{L}_{2}$, where $\mathcal{L}_{1}, \mathcal{L}_{2} \subseteq \mathcal{L}$. Let $L_{1}=\left|\mathcal{L}_{1}\right|$ and $L_{2}=\left|\mathcal{L}_{2}\right|$. We assume that the requests $\ell_{1}$ and $\ell_{2}$ are known by the server and both users during the delivery phase (this information can be shared, for example, with vanishing rate for finite $L$ and $n \rightarrow \infty$ ). A two-user $R D C$ code for a given blocklength $n$, which we call a two-user $\left(n, \mathcal{M}_{\mathrm{c}}^{(n)}, \mathcal{M}^{(n)}\right)$-code, consists of the following mappings:

(i) A cache-phase encoder at the server

$$
\phi_{\mathrm{c}}^{(n)}: \mathcal{X}^{n} \rightarrow \mathcal{M}_{\mathrm{c}}^{(n)}
$$

(ii) A delivery-phase encoder at the server

$$
\phi_{\left(\ell_{1} \ell_{2}\right)}^{(n)}: \mathcal{X}^{n} \rightarrow \mathcal{M}^{(n)}
$$

for each pair of user requests $\left(\ell_{1}, \ell_{2}\right) \in \mathcal{L}_{1} \times \mathcal{L}_{2}$.

(iii) A delivery-phase decoder at user 1

$$
\varphi_{\ell_{1} \ell_{2}}^{(n)}: \mathcal{M}^{(n)} \times \mathcal{M}_{\mathrm{c}}^{(n)} \rightarrow \hat{\mathcal{X}}_{\ell_{1}}^{n}
$$

for each pair of user requests $\left(\ell_{1}, \ell_{2}\right) \in \mathcal{L}_{1} \times \mathcal{L}_{2}$.

(iv) A delivery-phase decoder at user 2

$$
\psi_{\ell_{1} \ell_{2}}^{(n)}: \mathcal{M}^{(n)} \rightarrow \tilde{\mathcal{X}}_{\ell_{2}}^{n}
$$

for each pair of user requests $\left(\ell_{1}, \ell_{2}\right) \in \mathcal{L}_{1} \times \mathcal{L}_{2}$.

During the caching phase the server places

$$
M_{\mathrm{c}}^{(n)}=\phi_{\mathrm{c}}^{(n)}\left(\boldsymbol{X}^{n}\right)
$$

in the cache of user 1 . After the request of both users $\left(\ell_{1}, \ell_{2}\right) \in \mathcal{L}_{1} \times \mathcal{L}_{2}$ are revealed to the server and both users, the server sends

$$
M_{\ell_{1} \ell_{2}}^{(n)}=\phi_{\ell_{1} \ell_{2}}^{(n)}\left(\boldsymbol{X}^{n}\right)
$$

over a common noiseless channel to both users. Users 1 and 2 respectively output

$$
\hat{X}_{\ell_{1}}^{n}=\varphi_{\ell_{1} \ell_{2}}^{(n)}\left(M_{\ell_{1} \ell_{2}}^{(n)}, M_{\mathrm{c}}^{(n)}\right)
$$

and

$$
\tilde{X}_{\ell_{2}}^{n}=\psi_{\ell_{1} \ell_{2}}^{(n)}\left(M_{\ell_{1} \ell_{2}}^{(n)}\right) .
$$

The users might have differing exigencies regarding the sources in the library. To account for this, we allow for different single-symbol distortion functions at each user:

$$
\mathrm{d}_{\ell_{1}}: \hat{\mathcal{X}}_{\ell_{1}} \times \mathcal{X}_{\ell_{1}} \rightarrow[0, \infty) \quad(\text { user } 1)
$$

and

$$
\delta_{\ell_{2}}: \tilde{\mathcal{X}}_{\ell_{2}} \times \mathcal{X}_{\ell_{2}} \rightarrow[0, \infty) \quad(\text { user } 2)
$$

where $\mathrm{d}_{\ell_{1}}$ and $\delta_{\ell_{2}}$ satisfy the two conditions preceding Definition 1

Definition 10. We say that a rate-distortion-cache tuple $(R, \boldsymbol{D}, \boldsymbol{\Delta}, C)$-tuple is $(\mathbf{d}, \boldsymbol{\delta})$-achievable w.r.t. expected distortions if there exists a sequence of two-user $\left(n, \mathcal{M}_{c}^{(n)}, \mathcal{M}^{(n)}\right)$-codes such that

$$
\begin{gathered}
\limsup _{n \rightarrow \infty} \frac{1}{n} \log \left|\mathcal{M}_{c}^{(n)}\right| \leq C \\
\limsup _{n \rightarrow \infty} \frac{1}{n} \log \left|\mathcal{M}^{(n)}\right| \leq R
\end{gathered}
$$




$$
\begin{gathered}
\limsup _{n \rightarrow \infty} \mathbb{E}\left[\overline{\mathrm{d}}_{\ell_{1}}\left(\hat{X}_{\ell_{1}}^{n}, X_{\ell_{1}}^{n}\right)\right] \leq D_{\ell_{1}}, \quad \forall \ell_{1} \in \mathcal{L}_{1} \\
\limsup _{n \rightarrow \infty} \mathbb{E}\left[\bar{\delta}_{\ell_{2}}\left(\tilde{X}_{\ell_{2}}^{n}, X_{\ell_{2}}^{n}\right)\right] \leq \Delta_{\ell_{2}}, \quad \forall \ell_{2} \in \mathcal{L}_{2},
\end{gathered}
$$

where $\overline{\mathrm{d}}_{\ell_{1}}$ and $\bar{\delta}_{\ell_{2}}$ are the separable distortion functions (defined in the same way as (2)) corresponding to $\mathrm{d}_{\ell_{1}}$ and $\delta_{\ell_{2}}$. The two-user operational RDC function w.r.t. expected distortions $\mathrm{R}^{\dagger}(\boldsymbol{D}, \boldsymbol{\Delta}, C)$ is the infimum of all rates $R \geq 0$ such that the rate-distortion-cache tuple $(R, \boldsymbol{D}, \boldsymbol{\Delta}, C)$ is $(\mathbf{d}, \boldsymbol{\delta})$-achievable.

Unfortunately, we have been unable to find a single-letter (or, computable) expression for the two-user operational RDC function. An achievable (upper) bound and a converse (lower) bound are presented in the next two subsections.

\section{B. Achievable Bound}

As in the single-user problem, we would first like to jointly quantise the library $\boldsymbol{X}^{n}$ to a codeword $U^{n}$ that is placed in user 1's cache. (The manner in which $U^{n}$ is placed in the cache is described next.) After the requests $\ell_{1}$ and $\ell_{2}$ are revealed to the server and both users, we then would like to communicate the approximations $\hat{X}_{\ell_{1}}^{n}$ and $\tilde{X}_{\ell_{2}}^{n}$ to users 1 and 2 respectively.

Note that user 2 does not have a cache and, therefore, $\tilde{X}_{\ell_{2}}^{n}$ can always be recovered by user 1 . Indeed, user 1 will be able to reconstruct $\tilde{X}_{\ell_{2}}^{n}$ before it attempts to recover $U^{n}$ from its cache. We may, therefore, view $\tilde{X}_{\ell_{2}}^{n}$ as a type of side information at user 1 that should be exploited by the cache encoder at the server to reduce the caching rate.

For example, one could use a Wyner-Ziv-style code [20] to compress $\boldsymbol{X}^{n}$ to a description $U^{n}$ that is randomly binned at a rate matched to the bin size $I\left(U ; \tilde{X}_{\ell_{2}}\right)$. Unfortunately, the particular demand $\ell_{2}$ and, consequently, the bin size (or, binning rate) are not known to the server during the caching phase, and, for this reason, we need to use a slightly more sophisticated "implicit" binning technique to encode the cache. The technique is similar to that used by Tuncel in [21], and it leads to the following achievability result.

Let

$$
\overline{\mathrm{R}}(\boldsymbol{D}, \boldsymbol{\Delta}, C)=\min _{(U, \hat{\boldsymbol{X}}, \tilde{\boldsymbol{X}})\left(\ell_{1}, \ell_{2}\right)} \max _{\max }\left\{I\left(\boldsymbol{X} ; U, \hat{X}_{\ell_{1}}, \tilde{X}_{\ell_{2}}\right)-C, I\left(U, \boldsymbol{X} ; \tilde{X}_{\ell_{2}}\right)+I\left(\boldsymbol{X} ; \hat{X}_{\ell_{1}} \mid U, \tilde{X}_{\ell_{2}}\right),\right\}
$$

where the minimum is taken over all tuples $(U, \hat{\boldsymbol{X}}, \tilde{\boldsymbol{X}})$ jointly distributed with $\boldsymbol{X}$ such that

$$
\begin{array}{ll}
\forall \ell_{1} \in \mathcal{L}_{1}: & \mathbb{E}\left[\mathrm{d}_{\ell_{1}}\left(\hat{X}_{\ell_{1}}, X_{\ell_{1}}\right)\right] \leq D_{\ell_{1}} \\
\forall \ell_{2} \in \mathcal{L}_{2}: & \mathbb{E}\left[\delta_{\ell_{2}}\left(\hat{X}_{\ell_{2}}, X_{\ell_{2}}\right)\right] \leq \Delta_{\ell_{2}} .
\end{array}
$$

Theorem 26.

$$
\overline{\mathrm{R}}(\boldsymbol{D}, \boldsymbol{\Delta}, C) \geq \mathrm{R}^{\dagger}(\boldsymbol{D}, \boldsymbol{\Delta}, C) .
$$

with equality when $\mathcal{L}_{1}=\{\ell\}$ or $\mathcal{L}_{2}=\{\ell\}$ for some $\ell \in \mathcal{L}$.

Proof: An outline of the proof of Theorem 26 is given in Appendix $\mathrm{L}$.

\section{Genie-Aided Lower Bound}

If both users' demands were revealed by a genie to the server even before the caching phase, then the caching problem would coincide with a "worst-demands" of the classic successive-refinement of information problem [22, Sec. 13.5]. The RD function corresponding to this worst-demands problem thus forms a lower bound on $\mathrm{R}^{\dagger}(\boldsymbol{D}, \boldsymbol{\Delta}, C)$. More specifically, let

$$
\underline{\mathrm{R}}(\boldsymbol{D}, \boldsymbol{\Delta}, C)=\min _{(\hat{\boldsymbol{X}}, \tilde{\boldsymbol{X}})} \max _{\left(\ell_{1}, \ell_{2}\right)} \max \left\{I\left(\boldsymbol{X} ; \tilde{X}_{\ell_{2}}\right), I\left(\boldsymbol{X} ; \hat{X}_{\ell_{1}}, \tilde{X}_{\ell_{2}}\right)-C\right\}
$$

where the minimum is taken over all $(\hat{\boldsymbol{X}}, \tilde{\boldsymbol{X}})$ jointly distributed with $\boldsymbol{X}$ such that 28a and (28b) hold.

Theorem 27.

$$
\underline{\mathrm{R}}(\boldsymbol{D}, \boldsymbol{\Delta}, C) \leq \mathrm{R}^{\dagger}(\boldsymbol{D}, \boldsymbol{\Delta}, C) .
$$

D. (Almost) Lossless Reconstructions at both users

We now specialise Theorems 26 and 27 to the (almost) lossless reconstructions setup.

Corollary 28. For Hamming distortions

$$
\begin{aligned}
\mathrm{R}^{\dagger}(\mathbf{0}, \mathbf{0}, C) & \geq \underline{\mathrm{R}}(\mathbf{0}, \mathbf{0}, C) \\
& =\max _{\left(\ell_{1}, \ell_{2}\right)} \max \left\{H\left(X_{\ell_{2}}\right), H\left(X_{\ell_{1}}, X_{\ell_{2}}\right)-C\right\}
\end{aligned}
$$


and

$$
\begin{aligned}
\mathrm{R}^{\dagger}(\mathbf{0}, \mathbf{0}, C) & \leq \overline{\mathrm{R}}(\mathbf{0}, \mathbf{0}, C) \\
& =\min _{U} \max _{\left(\ell_{1}, \ell_{2}\right)} \max \left\{H\left(X_{\ell_{2}}\right)+H\left(X_{\ell_{1}} \mid U, X_{\ell_{2}}\right), H\left(U, X_{\ell_{1}}, X_{\ell_{2}}\right)-C\right\}
\end{aligned}
$$

where the minimisation is over all auxiliaries $U$. Moreover, 29] holds with equality when $\mathcal{L}_{1}=\{\ell\}$ for some $\ell \in \mathcal{L}$ or $\mathcal{L}_{1}=\mathcal{L}_{2}=\left\{\ell, \ell^{\prime}\right\}$ for $\ell, \ell^{\prime} \in \mathcal{L}$.

Interestingly, in these cases there is no penalty in the rate-distortions function even though the server does not know the users' demands during the caching phase.

\section{E. (Almost) Lossless Reconstruction at user 2}

We finally consider the setup where reconstruction at user 1 is lossy with a prescribed distortion, and reconstruction at user 2 is lossless $(\boldsymbol{\Delta}=\mathbf{0})$. We specialise Theorems 26, 27, and compare the obtained lower and upper bounds for a doubly symmetric binary source (DSBS). Then, we prove a stronger lower bound and show that it matches the upper bound of Theorem 26 for the studied DSBS.

Corollary 29. Theorems 26 and 27 provide the following upper and lower bound on the rate-distortion-memory function when $\Delta=0$ under the Hamming distortion function.

$$
\begin{aligned}
\mathrm{R}^{\dagger}(\boldsymbol{D}, \mathbf{0}, C) & \geq \underline{\mathrm{R}}(\boldsymbol{D}, \mathbf{0}, C) \\
& =\min _{\hat{\boldsymbol{X}}} \max _{\left(\ell_{1}, \ell_{2}\right)} H\left(X_{\ell_{2}}\right)+\max \left\{0, I\left(\boldsymbol{X} ; \hat{X}_{\ell_{1}} \mid X_{\ell_{2}}\right)-C\right\}
\end{aligned}
$$

and

$$
\begin{aligned}
R^{\dagger}(\boldsymbol{D}, \mathbf{0}, C) & \leq \overline{\mathrm{R}}(\boldsymbol{D}, \mathbf{0}, C) \\
& =\min _{(U, \hat{\boldsymbol{X}})} \max _{\left(\ell_{1}, \ell_{2}\right)} \max \left\{I\left(\boldsymbol{X} ; U, \hat{X}_{\ell_{1}}, X_{\ell_{2}}\right)-C, H\left(X_{\ell_{2}}\right)+I\left(\boldsymbol{X} ; \hat{X}_{\ell_{1}} \mid U, X_{\ell_{2}}\right),\right\}
\end{aligned}
$$

where the minimum is taken over all $(U, \hat{\boldsymbol{X}})$ jointly distributed with $\boldsymbol{X}$ such that (28a) holds.

For the case where the library consists of two files, which are the outcomes of a DSBS, we use Corollary 29 to find lower and upper bounds on the rate-distortion-cache function. The bounds meet for all distortions below a certain threshold.

Corollary 30. For the DSBS $\left(X_{\ell}, X_{\ell}^{\prime}\right)$ with parameter $\rho, 0 \leq \rho \leq \frac{1}{2}$, with symmetric distortion criteria $D_{\ell}=D_{\ell}^{\prime}=D$ under the Hamming distortion function, we have

$$
\mathrm{R}^{\dagger}(\boldsymbol{D}, \mathbf{0}, C) \geq 1+(h(\rho)-h(D)-C)^{+}
$$

and

$$
\begin{aligned}
& R^{\dagger}(\boldsymbol{D}, \mathbf{0}, C) \\
& \leq\left\{\begin{array}{l}
1+(h(\rho)-h(D)-C)^{+} \\
\text {If } D \leq D^{\star} \\
1+\left(h(D)-\rho-(1-\rho) h\left(\frac{2 D-\rho}{2(1-\rho)}\right)-C\right)^{+} \\
\text {If } D^{\star}<D \leq \frac{1}{2}
\end{array}\right.
\end{aligned}
$$

where

$$
D^{\star}=\frac{1}{2}(1-\sqrt{1-2 \rho)} .
$$

Proof: The proof is outlined in Appendix M, and uses connections to the problem of broadcasting to two users with complementary side information [19].

Finally, we find a lower bound on the delivery rate by considering an average-case scenario, rather than a worst-case scenario, for user 2. This type of lower bounds has also appeared in [31], [32], and [33].

Theorem 31. For any distribution $p_{I}($.$) on \left\{1, \ldots, L_{2}\right\}$, we have

$$
R^{\dagger}(\boldsymbol{D}, \mathbf{0}, C) \geq \min _{(U, \hat{\boldsymbol{X}})} \max _{\ell_{1}} \max \left\{I\left(\boldsymbol{X} ; U, \hat{X}_{\ell_{1}}, X_{I}\right)-C, H\left(X_{I} \mid I\right)+I\left(\boldsymbol{X} ; \hat{X}_{\ell_{1}} \mid U, X_{I}\right),\right\}
$$

where $X_{I}$ is indexed by the random variable $I$ and the minimisation is over all $p_{U \hat{\boldsymbol{X}} \mid \boldsymbol{X} I}$ such that

$$
p_{U \hat{\boldsymbol{X}} \mid \boldsymbol{X} I}=p_{\hat{\boldsymbol{X}} \mid \boldsymbol{X}} \times p_{U \mid \hat{\boldsymbol{X}} \boldsymbol{X} I}
$$


and 28a holds. To compute the above optimization, the cardinality of $\mathcal{U}$ may be restricted to $\mathcal{U} \leq|\mathcal{X}|+2 L_{1}$ without any loss of generality.

Proof: Theorem 31 is proved in Appendix $\mathrm{N}$

Remark 2. For symmetric sources where $p_{X_{1}}()=.\ldots=p_{X_{L}}($.$) , 40 reduces to$

$$
R^{\dagger}(\boldsymbol{D}, \mathbf{0}, C) \geq \min _{(U, \hat{\boldsymbol{X}})} \max _{\ell_{1}} \max \left\{I\left(\boldsymbol{X} ; U, \hat{X}_{\ell_{1}}, X_{I}\right)-C, H\left(X_{I}\right)+I\left(\boldsymbol{X} ; \hat{X}_{\ell_{1}} \mid U, X_{I}\right)\right\}
$$

Note that (42) looks similar to 35 but $X_{I}$ implies an average demand criterion at user 2 and may in general be weaker than (27).

Remark 3. The lower bound in Theorem 31 is larger than or equal to the genie-aided lower bound in Theorem 27

\section{APPENDIX A}

\section{PROOF OF THEOREM 3}

\section{A. Achievability (Expected and Excess Distortions)}

Pick any test channel $q_{U \mid \boldsymbol{X}}$ from $\mathcal{X}$ to $\mathcal{U}$ such that $(\boldsymbol{X}, U) \sim p_{\boldsymbol{X}} q_{U \mid \boldsymbol{X}}$ satisfies $I(\boldsymbol{X} ; U)<C$. Build a codebook for the cache by randomly generating $2^{n R^{\prime}}$ codewords

$$
\mathcal{C}_{\text {cache }}:=\left\{U^{n}(j)=\left(U_{1}(j), U_{2}(j), \ldots, U_{n}(j)\right)\right\}_{j=1}^{2^{n R^{\prime}}},
$$

each of length $n$, by selecting symbols iid $U \sim q_{U}$ from $\mathcal{U}$. Give $\mathcal{C}_{\text {cache }}$ to the server and user.

The server observes the entire library $\boldsymbol{X}^{n}$, and it looks for a unique codeword index $j$ such that $\left(\boldsymbol{X}^{n}, U^{n}(j)\right)$ is jointly typical (for example, we may use letter typicality [24] or strong typicality [25]). If successful, the transmitter places $j$ in the cache; if unsuccessful, the server places $j=1$ in the cache. If $R^{\prime}>I(\boldsymbol{X} ; U)$, then the probability of a cache encoding error (i.e., such a $j$ cannot be found) vanishes exponentially fast in the blocklength $n$.

The user reads the index $j$ from its cache, and it recovers the codeword $U^{n}(j)$ from the cache codebook $\mathcal{C}_{\text {cache. If the server's }}$ cache-encoding step was successful, then $U^{n}(j)$ will be jointly typical with $X_{\ell}^{n}$. The server-user compression problem then reduces to the standard conditional rate-distortion problem [10] with $U^{n}(j)$ as side information.

\section{B. Weak Converse (Expected Distortions)}

Fix the distortion tuple $\boldsymbol{D}$ and cache capacity $C$ arbitrarily, and suppose that $R$ is $(\boldsymbol{D}, C)$-admissible. Suppose that we have a sequence of $\left(n, \mathcal{M}, \mathcal{M}_{\mathrm{c}}^{(n)}\right)$-codes satisfying (5). For any $\varepsilon>0$ there exists a sufficiently large $n^{*}$ depending on $\epsilon$ such that for all $n \geq n^{*}$ we have

$$
\begin{aligned}
C+\varepsilon & \geq \frac{1}{n} \log \left|\mathcal{M}_{\mathrm{c}}^{(n)}\right| \\
& \geq \frac{1}{n} H\left(M_{\mathrm{c}}^{(n)}\right) \\
& \geq \frac{1}{n} I\left(\boldsymbol{X}^{n} ; M_{\mathrm{c}}^{(n)}\right) \\
& =\frac{1}{n} \sum_{i=1}^{n} I\left(\boldsymbol{X}_{i} ; M_{\mathrm{c}}^{(n)} \mid \boldsymbol{X}_{1}^{i-1}\right) \\
& \stackrel{\mathrm{a}}{=} \frac{1}{n} \sum_{i=1}^{n} I\left(\boldsymbol{X}_{i} ; M_{\mathrm{c}}^{(n)}, \boldsymbol{X}_{1}^{i-1}\right) \\
& \stackrel{\mathrm{b}}{=} \frac{1}{n} \sum_{i=1}^{n} I\left(\boldsymbol{X}_{i} ; U_{i}\right),
\end{aligned}
$$

where (a) follows because $\boldsymbol{X}^{n}$ is iid, and (b) follows by setting

$$
U_{i}=\left(M_{\mathrm{c}}^{(n)}, \boldsymbol{X}_{1}^{i-1}\right) \quad \text { on } \quad \mathcal{U}_{i}=\mathcal{M}_{\mathrm{c}}^{(n)} \times \mathcal{X}^{i-1} .
$$

Similarly, for each index $\ell \in \mathcal{L}$ and $n \geq n^{*}$ we have

$$
\begin{aligned}
R+\varepsilon & \geq \frac{1}{n} \log \left|\mathcal{M}^{(n)}\right| \\
& \geq \frac{1}{n} H\left(M_{\ell}^{(n)}\right)
\end{aligned}
$$




$$
\begin{aligned}
& \geq \frac{1}{n} I\left(\boldsymbol{X}^{n} ; M_{\ell}^{(n)} \mid M_{\mathrm{c}}^{(n)}\right) \\
& \geq \frac{1}{n} I\left(\boldsymbol{X}^{n} ; \hat{X}_{\ell}^{n} \mid M_{\mathrm{c}}^{(n)}\right) \\
& \geq \frac{1}{n} \sum_{i=1}^{n} I\left(\boldsymbol{X}_{i} ; \hat{X}_{\ell}^{n} \mid M_{\mathrm{c}}^{(n)}, \boldsymbol{X}_{1}^{i-1}\right) \\
& \stackrel{\mathrm{b}}{=} \frac{1}{n} \sum_{i=1}^{n} I\left(\boldsymbol{X}_{i} ; \hat{X}_{\ell}^{n} \mid U_{i}\right) \\
& \geq \frac{1}{n} \sum_{i=1}^{n} I\left(X_{\ell i} ; \hat{X}_{\ell i} \mid U_{i}\right)
\end{aligned}
$$

where (a) follows because $\hat{X}_{\ell}^{n}$ is a function of the delivery-phase message $M_{\ell}^{(n)}$ and the cached message $M_{\mathrm{c}}^{(n)}$ and (b) substitutes $U_{i}$.

Consider each index $i$ in the above sum and, in particular, the tuple $\left(\boldsymbol{X}_{i}, U_{i}, \hat{\boldsymbol{X}}_{i}\right)$. We can write the joint pmf of this tuple as

$$
p_{\boldsymbol{X}_{i} U_{i} \hat{\boldsymbol{X}}_{i}}\left(\boldsymbol{x}_{i}, u_{i}, \hat{\boldsymbol{x}}_{i}\right)=p_{\boldsymbol{X}}\left(\boldsymbol{x}_{i}\right) q_{U_{i} \mid \boldsymbol{X}_{i}}\left(u_{i} \mid \boldsymbol{x}_{i}\right) q_{\hat{\boldsymbol{X}}_{i} \mid \boldsymbol{X}_{i}, U_{i}}\left(\hat{\boldsymbol{x}}_{i} \mid \boldsymbol{x}_{i}, u_{i}\right)
$$

Let

$$
\mathcal{U}^{*}=\bigcup_{i=1}^{n} \mathcal{U}_{i}
$$

and construct a new joint pmf on $\tilde{p}_{\boldsymbol{X} U \hat{\boldsymbol{X}}}$ on $\mathcal{X} \times \mathcal{U} \times \hat{\mathcal{X}}$ by setting

$$
p_{\boldsymbol{X} U \hat{\boldsymbol{X}}}^{*}(\boldsymbol{x}, u, \hat{\boldsymbol{x}})=p_{\boldsymbol{X}}(\boldsymbol{x}) \frac{1}{n} \sum_{i=1}^{n} \mathbb{1}\left\{u \in \mathcal{U}_{i}\right\} q_{U_{i} \mid \boldsymbol{X}_{i}}\left(u_{i} \mid \boldsymbol{x}_{i}\right) q_{\hat{\boldsymbol{X}}_{i} \mid \boldsymbol{X}_{i}}\left(\hat{\boldsymbol{x}}_{i} \mid u, \boldsymbol{x}\right) .
$$

Then,

$$
\begin{aligned}
\frac{1}{n} \sum_{n=1}^{n} I\left(\boldsymbol{X}_{i} ; U_{i}\right) & =I_{p_{\boldsymbol{X} U \hat{\boldsymbol{X}}}^{*}}(\boldsymbol{X} ; U) \\
\frac{1}{n} \sum_{n=1}^{n} I\left(X_{\ell, i} ; \hat{X}_{\ell, i} \mid U_{i}\right) & =I_{p_{\boldsymbol{X} U \hat{\boldsymbol{X}}}^{*}}\left(X_{\ell} ; \hat{X}_{\ell} \mid U\right)
\end{aligned}
$$

and

$$
D_{\ell}+\epsilon \geq \mathbb{E}\left[\frac{1}{n} \sum_{i=1}^{n} \mathrm{~d}_{\ell}\left(\hat{X}_{\ell, i}, X_{\ell, i}\right)\right]=\mathbb{E}_{p_{X U \hat{\mathbf{X}}}^{*}}\left[\mathrm{~d}_{\ell}\left(\hat{X}_{\ell}, X_{\ell}\right)\right]
$$

for all $\ell \in \mathcal{L}$. To complete the converse, we need only show that the alphabet $\mathcal{U}^{*}$ and the joint pmf $p_{X U \hat{\boldsymbol{X}}}^{*}$ on $\mathcal{X} \times \mathcal{U}^{*} \times \hat{\mathcal{X}}$ can be replaced by an alphabet $\mathcal{U}$ of cardinality $|\mathcal{U}| \leq|\mathcal{X}|+2 L$ and a joint pmf $q_{\boldsymbol{X} U \hat{\boldsymbol{X}}}$ on $\mathcal{X} \times \mathcal{U} \times \hat{\mathcal{X}}$ such that

(i) $I_{p_{\boldsymbol{X} U \hat{\boldsymbol{X}}}^{*}}(\boldsymbol{X} ; U)=I_{q_{\boldsymbol{X} U \hat{\boldsymbol{X}}}}(\boldsymbol{X} ; U)$

(ii) $\forall \ell \in \mathcal{L}: I_{p_{X U \hat{\boldsymbol{X}}}^{*}}\left(X_{\ell} ; \hat{X}_{\ell} \mid U\right)=I_{q_{\boldsymbol{X} U \hat{\boldsymbol{X}}}}\left(X_{\ell} ; \hat{X}_{\ell} \mid U\right)$

(iii) $\forall \ell \in \mathcal{L}: \mathbb{E}_{p_{\boldsymbol{X} U \hat{\boldsymbol{X}}}^{*}}\left[\mathrm{~d}_{\ell}\left(\hat{X}_{\ell}, X_{\ell}\right)\right]=\mathbb{E}_{q_{\boldsymbol{X} U \hat{\boldsymbol{X}}}}\left[\mathrm{d}_{\ell}\left(\hat{X}_{\ell}, X_{\ell}\right)\right]$.

Let $q \in \mathcal{P}^{*}$ and $\boldsymbol{x}^{*} \in \mathcal{X}$ be arbitrary, and define the following functions.

1) For each $\boldsymbol{x} \in \mathcal{X}$, let

$$
g_{\boldsymbol{x}}^{1}(q):=\sum_{\hat{\boldsymbol{x}} \in \hat{\mathcal{X}}} q(\boldsymbol{x}, \hat{\boldsymbol{x}})
$$

2)

$$
g^{2}(q):=-\sum_{\boldsymbol{x} \in \mathcal{X}}\left(\sum_{\hat{\boldsymbol{x}} \in \hat{\mathcal{X}}} q(\boldsymbol{x}, \hat{\boldsymbol{x}})\right) \log \left(\sum_{\hat{\boldsymbol{x}} \in \hat{\mathcal{X}}} q(\boldsymbol{x}, \hat{\boldsymbol{x}})\right)
$$

3) For each $\ell \in \mathcal{L}$, let

$$
g_{\ell}^{3}(q):=\sum_{x_{\ell} \in \mathcal{X}_{\ell}} \sum_{\hat{x}_{\ell} \in \hat{\mathcal{X}}_{\ell}} q\left(x_{\ell}, \hat{x}_{\ell}\right) \cdot \log \frac{q\left(x_{\ell}, \hat{x}_{\ell}\right)}{\left(\sum_{\hat{x}_{\ell} \in \hat{\mathcal{X}}_{\ell}} q\left(x_{\ell}, \hat{x}_{\ell}\right)\right)\left(\sum_{x_{\ell} \in \mathcal{X}_{\ell}} q\left(x_{\ell}, \hat{x}_{\ell}\right)\right)}
$$


4) For each $\ell \in \mathcal{L}$, let

$$
g_{\ell}^{4}(q):=\sum_{x_{\ell} \in \mathcal{X}_{\ell}} \sum_{\hat{x}_{\ell} \in \hat{\mathcal{X}}_{\ell}} q\left(x_{\ell}, \hat{x}_{\ell}\right) \mathrm{d}_{\ell}\left(\hat{x}_{\ell}, x_{\ell}\right) .
$$

Define a Borel measure $\mu$ on $\mathcal{P}^{*}$ by

$$
\mu\left(p_{\mid U=u}^{*}\right):=p_{U}^{*}(u), \quad u \in \mathcal{U}^{*} .
$$

Then,

$$
\begin{aligned}
& \int g_{\boldsymbol{x}}^{1} d \mu=p_{\boldsymbol{X}}^{*}(\boldsymbol{x}), \quad \forall \boldsymbol{x} \in \mathcal{X} . \\
& \int g^{2} d \mu=H_{p_{\boldsymbol{X}, U}^{*}}(\boldsymbol{X} \mid U) \\
& \int g_{\ell}^{3} d \mu=I_{p_{\boldsymbol{X}, U, \hat{\boldsymbol{X}}}^{*}}\left(X_{\ell} ; \hat{X}_{\ell} \mid U\right), \quad \forall \ell \in \mathcal{L} . \\
& \int g_{\ell}^{4} d \mu=\mathbb{E}_{p_{X_{\ell}, U, \hat{X}_{\ell}}^{*}} \mathrm{~d}_{\ell}\left(\hat{X}_{\ell}, X_{\ell}\right), \quad \forall \ell \in \mathcal{L} .
\end{aligned}
$$

By Carathéodory's theorem, it is possible to assign probabilities to at most $|\mathcal{X}|+2 L$ points in $\mathcal{U}^{*}$ and preserve the area of the above functions.

\section{Weak Converse (Excess Distortions)}

The weak converse for excess distortions follows immediately from Assertion IV of Lemma 2 and the weak converse for expected distortions given above.

\section{APPENDIX B}

PROOF OF THEOREM 6

\section{A. Proof of Theorem 6}

Choose the cache capacity to be $C=\mathrm{C}_{\mathrm{g}}(\boldsymbol{D})$ and assume that $\mathrm{R}\left(\boldsymbol{D}, \mathrm{C}_{\mathrm{g}}(\boldsymbol{D})\right)>0$. By the definition of $\mathrm{C}_{\mathrm{g}}(\boldsymbol{D})$ :

$$
\mathrm{R}(\boldsymbol{D}, C)=\max _{\ell \in \mathcal{L}} \mathrm{R}_{X_{\ell}}\left(D_{\ell}\right)-C .
$$

Let $U$ be an optimal auxiliary random variable for the informational $\operatorname{RDC}$ function $\operatorname{R}(\boldsymbol{D}, C)$, i.e., $U$ is so that

$$
\mathrm{R}(\boldsymbol{D}, C)=\max _{\ell \in \mathcal{L}} \mathrm{R}_{X_{\ell} \mid U}\left(D_{\ell}\right)
$$

and

$$
I(\boldsymbol{X} ; U) \leq C
$$

Let $\ell^{*} \in \mathcal{L}^{*}$, i.e., $\ell^{*}$ attains the maximum in 43 . We have the following:

$$
\begin{aligned}
& \mathrm{R}(\boldsymbol{D}, C) \stackrel{\mathrm{a}}{=} \max _{\ell \in \mathcal{L}} \mathrm{R}_{X_{\ell} \mid U}\left(D_{\ell}\right) \\
& \geq \mathrm{R}_{X_{\ell^{*}} \mid U}\left(D_{\ell^{*}}\right) \\
& =\min _{q_{\hat{X}_{\ell^{*}} \mid X_{\ell^{*}}, U}: \mathbb{E}\left[d\left(\hat{X}_{\ell^{*}}, X_{\ell^{*}}\right)\right] \leq D_{\ell}} I\left(X_{\ell^{*}} ; \hat{X}_{\ell^{*}} \mid U\right) \\
& \stackrel{\mathrm{b}}{\geq} \min _{q_{\hat{X}_{\ell^{*}} \mid X_{\ell^{*}, U}}: \mathbb{E}\left[d\left(\hat{X}_{\ell^{*}}, X_{\ell^{*}}\right] \leq D_{\ell}\right.} I\left(X_{\ell^{*}} ; U, \hat{X}_{\ell^{*}}\right)-I(\boldsymbol{X} ; U) \\
& \stackrel{\mathrm{c}}{\geq} \mathrm{R}_{X_{\ell^{*}}}\left(D_{\ell^{*}}\right)-I(\boldsymbol{X} ; U) \\
& \stackrel{\mathrm{d}}{\geq} \mathrm{R}_{X_{\ell^{*}}}\left(D_{\ell^{*}}\right)-C \\
& \stackrel{\mathrm{e}}{=} \mathrm{R}(\boldsymbol{D}, C) \text {, }
\end{aligned}
$$

where (a) is identical to (44); (b) follows by adding the negative term $I\left(X_{\ell^{*}} ; U\right)-I(\boldsymbol{X} ; U) ;\left(\right.$ c) holds because $I\left(X_{\ell^{*}} ; U, \hat{X}_{\ell^{*}}\right) \geq$ $I\left(X_{\ell^{*}} ; \hat{X}_{\ell^{*}}\right)$; (d) holds by (45); and (e) holds by (43) and because $\ell^{*} \in \mathcal{L}^{*}$. 
The above inequalities must all hold with equality and so the chosen $U$ must satisfy $I(\boldsymbol{X} ; U)=C=\mathrm{C}_{\mathrm{g}}(\boldsymbol{D}), 9 \mathrm{a}$ ) and $9 \mathrm{~b}$. Therefore,

$$
\mathrm{C}_{\mathrm{g}}(\boldsymbol{D}) \leq \mathrm{C}_{\mathrm{g}}^{*}(\boldsymbol{D})
$$

Choose now the cache capacity $C=\mathrm{C}_{\mathrm{g}}^{*}(\boldsymbol{D})$, and let $U$ be an optimal auxiliary random variable for $\mathrm{C}_{\mathrm{g}}^{*}(\boldsymbol{D})$. That means, $U$ satisfies $9 \mathrm{a}$ and $9 \mathrm{~b}$ and

$$
I(\boldsymbol{X} ; U)=\mathrm{C}_{\mathrm{g}}^{*}(\boldsymbol{D})=C
$$

The following holds for all $\ell^{*} \in \mathcal{L}^{*}$ :

$$
\begin{aligned}
\mathrm{R}(\boldsymbol{D}, C) & \stackrel{\mathrm{a}}{\leq} \max _{\ell \in \mathcal{L}} \mathrm{R}_{X_{\ell} \mid U}\left(D_{\ell}\right) \\
& \stackrel{\mathrm{b}}{=} \mathrm{R}_{X_{\ell^{*}} \mid U}\left(D_{\ell^{*}}\right) \\
& \stackrel{\mathrm{c}}{=} \mathrm{R}_{X_{\ell^{*}}}\left(D_{\ell^{*}}\right)-I(\boldsymbol{X} ; U) \\
& \stackrel{\mathrm{d}}{=} \mathrm{R}_{X_{\ell^{*}}}\left(D_{\ell^{*}}\right)-C,
\end{aligned}
$$

where (a) follows because $U$ need not be optimal for $\mathrm{R}(\boldsymbol{D}, C)$, (b) follows from (9b), (c) follows from 9a), and (d) from (47).

Therefore, at the cache capacity $C=I(\boldsymbol{X} ; U)=\mathrm{C}_{\mathrm{g}}^{*}(\boldsymbol{D})$ we have $\mathrm{R}(D, C)=\mathrm{R}_{X_{\ell^{*}}}\left(D_{\ell^{*}}\right)-C$ and consequently

$$
\mathrm{C}_{\mathrm{g}}(\boldsymbol{D}) \geq \mathrm{C}_{\mathrm{g}}^{*}(\boldsymbol{D})
$$

The theorem follows from 46) and 48.

\section{B. Proof of Corollary 7}

The conditional RD function particularises to the conditional entropy function: $\mathrm{R}_{X_{\ell} \mid U}(0)=H\left(X_{\ell} \mid U\right)$. Similarly, the constraint 9a particularises to

$$
I(\boldsymbol{X} ; U)=H\left(X_{\ell^{*}}\right)-H\left(X_{\ell^{*}} \mid U\right)=I\left(X_{\ell^{*}} ; U\right),
$$

which is equivalent to $U \leftrightarrow X_{\ell^{*}} \leftrightarrow X_{\mathcal{L} \backslash \ell^{*}}$.

\section{APPENDIX C}

PROOF OF THEOREM 8

Let $\ell \in \mathcal{L}$. For any $(\boldsymbol{X}, U) \sim p_{\boldsymbol{X}} p_{U \mid \boldsymbol{X}}$ on $\mathcal{X} \times \mathcal{U}$, the following inequalities hold:

$$
\begin{aligned}
\mathrm{R}_{\boldsymbol{X}_{\ell} \mid U}\left(D_{\ell}\right) & =\min _{q_{\hat{X}_{\ell}^{\prime} \mid U, X_{\ell}}: \mathbb{E}\left[\mathrm{d}_{\ell}\left(\hat{X}_{\ell}^{\prime}, X_{\ell}\right)\right] \leq D_{\ell}} I\left(X_{\ell} ; \hat{X}_{\ell}^{\prime} \mid U\right) \\
& \geq \min _{q_{\hat{X}_{\ell}^{\prime} \mid X_{\ell}}: \mathbb{E}\left[\mathrm{d}_{\ell}\left(\hat{X}_{\ell}^{\prime}, X_{\ell}\right)\right] \leq D_{\ell}} I\left(X_{\ell} ; \hat{X}_{\ell}^{\prime}\right)-I(\boldsymbol{X} ; U) \\
& =\mathrm{R}_{X_{\ell}}\left(D_{\ell}\right)-I(\boldsymbol{X} ; U) .
\end{aligned}
$$

Now suppose that we have $(U, \hat{\boldsymbol{X}}) \sim p_{\hat{\boldsymbol{X}}, U \mid \boldsymbol{X}}$ on $\mathcal{U} \times \hat{\mathcal{X}}$ satisfying conditions (i), (ii), (iii), and (iv) in Definition 5 . Then,

$$
\begin{aligned}
\mathrm{R}_{X_{\ell} \mid U}\left(D_{\ell}\right) & \stackrel{\mathrm{a}}{\leq} I\left(X_{\ell} ; \hat{X}_{\ell} \mid U\right) \\
& \stackrel{\mathrm{b}}{=} I\left(X_{\ell} ; \hat{X}_{\ell}\right)-I(\boldsymbol{X} ; U) \\
& \stackrel{\mathrm{c}}{=} \mathrm{R}_{X_{\ell}}\left(D_{\ell}\right)-I(\boldsymbol{X} ; U)
\end{aligned}
$$

where (a) follows from property (iii) of Definition 5, (b) follows by properties (i) and (ii) of Definition 5, and (c) follows from property (iv) of Definition 5 .

Inequalities (49) and (50) combine to

$$
\mathrm{R}_{X_{\ell} \mid U}\left(D_{\ell}\right)=\mathrm{R}_{X_{\ell}}\left(D_{\ell}\right)-I(\boldsymbol{X} ; U), \quad \forall \ell \in \mathcal{L} .
$$

Thus, the pair $(U, \hat{\boldsymbol{X}})$ satisfies $(9 \mathrm{a})$. Moreover, since the mutual information $I(\boldsymbol{X} ; U)$ does not depend on $\ell \in \mathcal{L}$, the conditional rate-distortion function $\mathrm{R}_{X_{\ell} \mid U}\left(D_{\ell}\right)$ is largest for the same indices $\ell$ as the standard rate-distortion function $\mathrm{R}_{X_{\ell}}\left(D_{\ell}\right)$. Since $\mathrm{R}_{X_{\ell}}\left(D_{\ell}\right)$ is maximum for indices $\ell^{*} \in \mathcal{L}^{*}$, this proves that the pair $(U, \hat{\boldsymbol{X}})$ also satisfies $(9 \mathrm{~b})$. To conclude: If $(U, \hat{\boldsymbol{X}})$ satisfies (i), (ii), (iii), and (iv) in Definition 5, then $U$ is a valid tuple for $\mathrm{C}_{\mathrm{g}}^{*}(\boldsymbol{D})$ and $\mathrm{K}_{\mathrm{GK}}(\boldsymbol{D}) \leq \mathrm{C}_{\mathrm{g}}^{*}(\boldsymbol{D})$.

Now suppose that

$$
\mathrm{R}_{X_{1}}\left(D_{1}\right)=\mathrm{R}_{X_{2}}\left(D_{2}\right)=\cdots=\mathrm{R}_{X_{L}}\left(D_{L}\right)
$$


and, therefore, $\mathcal{L}^{*}=\mathcal{L}$. Let $U \sim p_{U \mid X}$ on $\mathcal{U}$ be any auxiliary random variable satisfying $9 \mathrm{a}$ ) for every $\ell \in \mathcal{L}$. (Condition $9 \mathrm{~b}$ automatically follows because $\mathcal{L}^{*}=\mathcal{L}$.) For each $\ell \in \mathcal{L}$, let $p_{\hat{X}_{\ell} \mid U X_{\ell}}$ be any test channel that is optimal for the informational conditional RD function

$$
\mathrm{R}_{X_{\ell} \mid U}\left(D_{\ell}\right)=\min _{q_{\hat{X}_{\ell}^{\prime} \mid U X_{\ell}}: \mathbb{E}\left[\mathrm{d}_{\ell}\left(\hat{X}_{\ell}^{\prime}, X_{\ell}\right)\right] \leq D_{\ell}} I\left(X_{\ell} ; \hat{X}_{\ell}^{\prime} \mid U\right) .
$$

Now consider the tuple

$$
(\boldsymbol{X}, U, \hat{\boldsymbol{X}}) \sim p_{\boldsymbol{X}} p_{U \mid \boldsymbol{X}} \prod_{\ell \in \mathcal{L}} p_{\hat{X}_{\ell} \mid U X_{\ell}}
$$

For all $\ell \in \mathcal{L}$ we have

$$
\begin{aligned}
\mathrm{R}_{X_{\ell} \mid U}\left(D_{\ell}\right) & \stackrel{\mathrm{a}}{=} \mathrm{R}_{X_{\ell}}\left(D_{\ell}\right)-I(\boldsymbol{X} ; U) \\
& \stackrel{\mathrm{b}}{\leq} I\left(X_{\ell} ; \hat{X}_{\ell}\right)-I(\boldsymbol{X} ; U) \\
& \leq I\left(X_{\ell} ; \hat{X}_{\ell} \mid U\right) \\
& \stackrel{\mathrm{c}}{=} \mathrm{R}_{X_{\ell} \mid U}\left(D_{\ell}\right),
\end{aligned}
$$

where (a) follow because $U$ was originally chosen to satisfy 9 9a); (b) follows because $(\boldsymbol{X}, U, \hat{\boldsymbol{X}})$ need not be optimal for the informational RD functions $\mathrm{R}_{X_{\ell}}\left(D_{\ell}\right)$; and (c) follows because $p_{\hat{X}_{\ell} \mid U X_{\ell}}$ achieves $\mathrm{R}_{X_{\ell} \mid U}\left(D_{\ell}\right)$. The above inequalities must be equalities and, therefore, $(\boldsymbol{X}, U, \hat{\boldsymbol{X}})$ satisfies the following four conditions:

- $\forall \ell \in \mathcal{L}: U \leftrightarrow X_{\ell} \leftrightarrow X_{\mathcal{L} \backslash \ell}$

- $\forall \ell \in \mathcal{L}: U \leftrightarrow \hat{X}_{\ell} \leftrightarrow X_{\ell}$

- $\forall \ell \in \mathcal{L}: I\left(X_{\ell} ; \hat{X}_{\ell}\right)=\mathrm{R}_{X_{\ell}}\left(D_{\ell}\right)$

- $\forall \ell \in \mathcal{L}: \mathbb{E}\left[\mathrm{d}_{\ell}\left(\hat{X}_{\ell}, X_{\ell}\right)\right] \leq D_{\ell}$.

To conclude: Given any $(\boldsymbol{X}, U) \sim p_{\boldsymbol{X}} p_{U \mid \boldsymbol{X}}$ satisfying 9a for all $\ell \in \mathcal{L}$ we can always find a test channel $p_{\hat{\boldsymbol{X}} \mid U \boldsymbol{X}}$ such that $(\boldsymbol{X}, U, \hat{\boldsymbol{X}}) \sim p_{\boldsymbol{X}} p_{U \mid \boldsymbol{X}} p_{\hat{\boldsymbol{X}} \mid U \boldsymbol{X}}$ satisfies the conditions of Definition 5

\section{APPENDIX D}

PROOF OF LEMMa 9

For Hamming distortion functions, it is not too hard to see that Definition 5 particularises to

$$
\mathrm{K}_{\mathrm{GK}}(\mathbf{0})=\max _{U: U \leftrightarrow X_{\ell} \leftrightarrow X_{\mathcal{L} \backslash \ell,}, \forall \ell \in \mathcal{L}} I(\boldsymbol{X} ; U) .
$$

Clearly, we have

$$
\max _{U: H\left(U \mid X_{\ell}\right)=0, \forall \ell \in \mathcal{L}} H(U) \leq \max _{U: U \leftrightarrow X_{\ell} \leftrightarrow X_{\mathcal{L} \backslash \ell}, \forall \ell \in \mathcal{L}} I(\boldsymbol{X} ; U)
$$

since any $U$ satisfying $H\left(U \mid X_{\ell}\right)=0$ for all $\ell \in \mathcal{L}$ must also satisfy $U \leftrightarrow X_{\ell} \leftrightarrow X_{\mathcal{L} \backslash \ell}$ for all $\ell \in \mathcal{L}$. The reverse inequality (and therefore Lemma 9] follows by the next lemma, which is a multivariate extension of [26, Lem. A.1].

Lemma 32. If $U$ is jointly distributed with $X$ such that $U \leftrightarrow X_{\ell} \leftrightarrow X_{\mathcal{L} \backslash \ell}$ for all $\ell \in \mathcal{L}$, then there exists $U^{\prime}$ jointly distributed with $(U, \boldsymbol{X})$ such that $U \leftrightarrow U^{\prime} \leftrightarrow \boldsymbol{X}$ and $H\left(U^{\prime} \mid X_{\ell}\right)=0$ for all $\ell \in \mathcal{L}$.

Proof: Let $p_{U \mid \boldsymbol{X}}$ denote the conditional distribution of $U$ given $\boldsymbol{X}$, and suppose that

$$
U \leftrightarrow X_{\ell} \leftrightarrow X_{\mathcal{L} \backslash \ell}, \quad \forall \ell \in \mathcal{L} .
$$

We first generate an $L$-partite graph

$$
\mathcal{G}=(\mathcal{V}, \mathcal{E})
$$

with vertices

$$
\mathcal{V}=\bigcup_{\ell \in \mathcal{L}} \mathcal{X}_{\ell}
$$

The edge set $\mathcal{E}$ contains an edge

$$
\left\{x, x^{\prime}\right\}, \quad x \in \mathcal{X}_{i}, x^{\prime} \in \mathcal{X}_{j}, \quad i, j \in \mathcal{L} \text { with } i \neq j,
$$

if and only if there exists an $\tilde{\boldsymbol{x}} \in \mathcal{X}$ with $\tilde{x}_{i}=x$ and $\tilde{x}_{j}=x^{\prime}$ and $p_{\boldsymbol{X}}(\tilde{\boldsymbol{x}})>0$.

Let $\mathcal{C}_{1}, \mathcal{C}_{2}, \ldots, \mathcal{C}_{N_{\mathrm{cc}}}$ denote the connected components of $\mathcal{G}$, and let $c(x)$ denote the index of the connected component that contains vertex $x$. 
Let us now construct a new auxiliary random variable $U^{\prime}$ on $\left\{1, \ldots, N_{\mathrm{cc}}\right\}$ that is jointly distributed with $\boldsymbol{X}$ by setting

$$
U^{\prime}=c\left(X_{1}\right) \text {. }
$$

Now, for any $\boldsymbol{x} \in \mathcal{X}$ with $p_{\boldsymbol{X}}(\boldsymbol{x})>0$, the corresponding set of vertices $\left\{x_{1}, \ldots, x_{L}\right\}$ forms a clique and, therefore, is a subgraph of some connected component. Therefore,

$$
U^{\prime}=c\left(X_{\ell}\right) \quad \text { a.s., } \forall \ell \in\{2, \ldots, L\} .
$$

This, of course, implies $H\left(U^{\prime} \mid X_{\ell}\right)=0$ for all $\ell$.

To complete the proof, we need only to show that $U$ can be generated by some conditional distribution $q_{U \mid U^{\prime}}:\{1, \ldots$, $\left.N_{\text {cc }}\right\} \rightarrow \mathcal{U}$. We first notice that the Markov chain (52) is equivalent to the following condition: For all $\boldsymbol{x} \in \mathcal{X}$ with $p_{\boldsymbol{X}}(\boldsymbol{x})>0$, we have

$$
p_{U \mid \boldsymbol{X}}(u \mid \boldsymbol{x})=p_{U \mid X_{1}}\left(u \mid x_{1}\right)=\cdots=p_{U \mid X_{L}}\left(u \mid x_{L}\right), \quad \forall u \in \mathcal{U} .
$$

Now consider any connected component $\mathcal{C}_{i}$ and any $u \in \mathcal{U}$. By the above method of constructing $\mathcal{G}$, we may conclude that

$$
p_{U \mid X_{\ell}}\left(u \mid x_{\ell}\right)=\text { constant, } \quad \forall \ell \in \mathcal{L} \text { and } x_{\ell} \in \mathcal{C}_{i} \cap \mathcal{X}_{\ell}
$$

That is, $p_{U \mid X_{\ell}}\left(u \mid x_{\ell}\right)$ depends only on the connected component $c\left(x_{\ell}\right)$ and the particular $u \in \mathcal{U}$, and we can write the above constant as $q_{c\left(x_{\ell}\right)}(u)$. Choose $p_{U \mid U^{\prime}}\left(u \mid u^{\prime}\right):=q_{u^{\prime}}(u)$ to complete the proof.

\section{ApPEndix E \\ PROOF OF THEOREM 12}

Choose the cache capacity $C=\mathrm{C}_{\mathrm{s}}(\boldsymbol{D})$. Let $U$ be an optimal auxiliary random variable for the informational RDC function; that is,

$$
\mathrm{R}(\boldsymbol{D}, C)=\max _{\ell \in \mathcal{L}} \mathrm{R}_{X_{\ell} \mid U}\left(D_{\ell}\right)
$$

Now, for each $\ell \in \mathcal{L}$, let $p_{\hat{X}_{\ell} \mid U}$ be an optimal test channel for the informational conditional RD function $\mathrm{R}_{X_{\ell} \mid U}\left(D_{\ell}\right)$. Define

$$
(\boldsymbol{X}, U, \hat{\boldsymbol{X}}) \sim p_{\boldsymbol{X}} p_{U \mid \boldsymbol{X}} \prod_{\ell \in \mathcal{L}} p_{\hat{X}_{\ell} \mid U X_{\ell}}
$$

and note that

$$
\hat{X}_{\ell} \leftrightarrow\left(U, X_{\ell}\right) \leftrightarrow\left(X_{\mathcal{L} \backslash \ell}, \hat{X}_{\mathcal{L} \backslash \ell}\right), \quad \forall \ell \in L
$$

Then,

$$
\begin{aligned}
\mathrm{R}(\boldsymbol{D}, C) & =\max _{\ell \in \mathcal{L}} I\left(X_{\ell} ; \hat{X}_{\ell} \mid U\right) \\
& \geq \frac{1}{L} \sum_{\ell=1}^{L} I\left(X_{\ell} ; \hat{X}_{\ell} \mid U\right) \\
& \geq \frac{1}{L} \sum_{\ell=1}^{L} I\left(\boldsymbol{X} ; \hat{X}_{\ell} \mid U, \hat{X}_{1}^{\ell-1}\right) \\
& =\frac{1}{L} I(\boldsymbol{X} ; \hat{\boldsymbol{X}} \mid U) \\
& \geq \frac{1}{L}\left(I(\boldsymbol{X} ; \hat{\boldsymbol{X}})-\mathrm{C}_{\mathrm{s}}(\boldsymbol{D})\right) \\
& \stackrel{\mathrm{c}}{\geq} \frac{1}{L}\left(\mathrm{R} \boldsymbol{X}(\boldsymbol{D})-\mathrm{C}_{\mathrm{s}}(\boldsymbol{D})\right) \\
& \stackrel{\mathrm{d}}{=} \mathrm{R}(\boldsymbol{D}, C),
\end{aligned}
$$

where (a) follows from 53 ; (b) follows because $I(\boldsymbol{X} ; U) \leq \mathrm{C}_{\mathbf{s}}(\boldsymbol{D})$; (c) follows because $\mathbb{E}\left[\mathrm{d}_{\ell}\left(X_{\ell}, \hat{X}_{\ell}\right)\right] \leq D_{\ell}$; and $(d)$ follows from the definition of $\mathrm{C}_{\mathrm{s}}(\boldsymbol{D})$.

The above inequalities are equalities and consequently $I\left(X_{1} ; \hat{X}_{1} \mid U\right)=\cdots=I\left(X_{L} ; \hat{X}_{L} \mid U\right), \hat{X}_{\ell} \leftrightarrow U \leftrightarrow \hat{X}_{\ell-1}$ (and therefore $\hat{X}_{\ell} \leftrightarrow U \leftrightarrow \hat{X}_{\mathcal{L} \backslash \ell}$ since the chain rule expansion order is arbitrary), $\boldsymbol{X} \leftrightarrow \hat{\boldsymbol{X}} \leftrightarrow U$ and $C=I(\boldsymbol{X} ; U)$. We can thus conclude that the tuple $(\boldsymbol{X}, U, \hat{\boldsymbol{X}})$ satisfies conditions (i)-(v) in the definition of $\mathrm{C}_{\mathrm{s}}^{*}(\boldsymbol{D})$ and $\mathrm{C}_{\mathrm{s}}^{*}(\boldsymbol{D}) \leq \mathrm{C}_{\mathrm{s}}(\boldsymbol{D})$.

Now suppose that $(\boldsymbol{X}, U, \hat{\boldsymbol{X}})$ satisfies conditions (i)-(v) in the definition of $\mathrm{C}_{\mathrm{s}}^{*}(\boldsymbol{D})$ and $I(\boldsymbol{X} ; U)=\mathrm{C}_{\mathrm{s}}^{*}(\boldsymbol{D})$. Then

$$
\mathrm{R}(\boldsymbol{D}, C) \leq \max _{\ell \in \mathcal{L}} I\left(X_{\ell} ; \hat{X}_{\ell} \mid U\right)
$$




$$
\begin{aligned}
& \stackrel{\mathrm{a}}{=} \frac{1}{L} \sum_{\ell=1}^{L} I\left(X_{\ell} ; \hat{X}_{\ell} \mid U\right) \\
& \stackrel{\mathrm{b}}{\leq} \frac{1}{L} \sum_{\ell=1}^{L} I\left(\boldsymbol{X} ; \hat{X}_{\ell} \mid U, \hat{X}_{1}^{\ell-1}\right) \\
& =\frac{1}{L}(I(\boldsymbol{X} ; \hat{\boldsymbol{X}}, U)-I(\boldsymbol{X} ; U)) \\
& \stackrel{\mathrm{c}}{\leq} \frac{1}{L}\left(\mathrm{R}_{\boldsymbol{X}}(\boldsymbol{D})-\mathrm{C}_{\mathrm{s}}^{*}(\boldsymbol{D})\right),
\end{aligned}
$$

where (a) follows because from condition (ii); (b) follows from condition (iii); (c) follows from conditions (i) and (v). Thus, we can achieve the superuser bound at $C=\mathrm{C}_{\mathrm{s}}^{*}(\boldsymbol{D})$ and $\mathrm{C}_{\mathrm{s}}^{*}(\boldsymbol{D}) \leq \mathrm{C}_{\mathrm{s}}(\boldsymbol{D})$.

\section{APPENDIX F}

PROOF OF LEMMA 15

First suppose that $\mathcal{S}=\{1,2, \ldots, S\}$ for some $1 \leq S \leq L$. Then

$$
D_{\mathcal{S}}=\left(D_{1}, \ldots, D_{S}\right) \text { and } \quad X_{\mathcal{S}}=\left(X_{1} \ldots, X_{S}\right)
$$

Let

$$
\mathrm{R}_{X_{\mathcal{S}}}\left(D_{\mathcal{S}}\right)=\min _{p_{\hat{X}_{\mathcal{S}} \mid X_{\mathcal{S}}}: \mathbb{E}\left[\mathrm{d}_{\ell}\left(\hat{X}_{\ell}, X_{\ell}\right)\right] \leq \mathrm{d}_{\ell}, \forall \ell \in \mathcal{L}} I\left(X_{\mathcal{S}} ; \hat{X}_{\mathcal{S}}\right)
$$

denote the joint RD function of the source $X_{\mathcal{S}}$ w.r.t. the $S$ distortion functions $\mathrm{d}_{1}, \mathrm{~d}_{2}, \ldots, \mathrm{d}_{S}$. A proof of the next lemma is essentially given in [27, Thm. 3.1] and is omitted.

Lemma 33. The joint RD function is upper bounded by

$$
\mathrm{R}_{X_{\mathcal{S}}}\left(D_{\mathcal{S}}\right) \leq \sum_{\ell=1}^{S} \mathrm{R}_{X_{\ell} \mid X_{1}^{\ell-1}}\left(D_{\ell}\right)
$$

where

$$
\mathrm{R}_{X_{\ell} \mid X_{1}^{\ell-1}}\left(D_{\ell}\right)=\min _{p_{\hat{X}_{\ell} \mid X_{1}^{\ell}}: \mathbb{E d}_{\ell}\left(\hat{X}_{\ell}, X_{\ell}\right) \leq D_{\ell}} I\left(X_{\ell} ; \hat{X}_{\ell} \mid X_{1}^{\ell-1}\right)
$$

denotes the conditional RD function for compressing a source $X_{\ell}$ with side information $X_{1}^{\ell-1}$.

Let us now return to Lemma 15 . We first notice that the information RDC function can be written as

$$
\mathrm{R}(\boldsymbol{D}, C)=\min _{p_{\hat{\boldsymbol{X}} U \mid \boldsymbol{X}} \in \mathcal{P}_{C}(\boldsymbol{D})} \max _{\ell \in \mathcal{L}} I\left(X_{\ell} ; \hat{X}_{\ell} \mid U\right),
$$

where $\mathcal{P}_{C}(\boldsymbol{D})$ denotes the set of all test channels $p_{\hat{\boldsymbol{X}} U \mid \boldsymbol{X}}$ from $\mathcal{X}$ to $\hat{\mathcal{X}} \times \mathcal{U}$ satisfying the cache-capacity constraint

$$
I(\boldsymbol{X} ; U) \leq C
$$

the expected-distortion constraints

$$
\mathbb{E}\left[\mathrm{d}_{\ell}\left(\hat{X}_{\ell}, X_{\ell}\right)\right] \leq \mathrm{d}_{\ell}, \quad \forall \ell \in \mathcal{L}
$$

and the Markov chains

$$
\hat{X}_{\ell} \leftrightarrow\left(X_{\ell}, U\right) \leftrightarrow\left(X_{\mathcal{L} \backslash \ell}, \hat{X}_{\mathcal{L} \backslash \ell}\right), \quad \forall \ell \in \mathcal{L} .
$$

Intuitively, the Markov chains in 57) can be imposed without changing the minimisation because each conditional mutual information $I\left(X_{\ell} ; \hat{X}_{\ell} \mid U\right)$ depends only on the marginal distribution of $\left(X_{\ell}, U, \hat{X}_{\ell}\right)$. Moreover, the minimum in (54) exists because the random variables are all defined on finite alphabets and conditional mutual information is continuous in $p_{\hat{\boldsymbol{X}} U \mid \boldsymbol{X}}$ and bounded from below. Pick any test channel that achieves this minimum and, with a slight abuse of notation, let $(\boldsymbol{X}, U, \hat{\boldsymbol{X}})$ denote the resulting tuple of random variables. We then have

$$
\begin{aligned}
& \mathrm{R}(\boldsymbol{D}, C) \geq \max _{\ell \in \mathcal{S}} I\left(X_{\ell} ; \hat{X}_{\ell} \mid U\right) \\
& \geq \frac{1}{S} \sum_{\ell=1}^{S} I\left(X_{\ell} ; \hat{X}_{\ell} \mid U\right) \\
& \geq \frac{1}{S} \sum_{\ell=1}^{S} I\left(X_{\ell} ; \hat{X}_{\ell} \mid U, X_{1}^{\ell-1}\right)
\end{aligned}
$$




$$
\begin{aligned}
& \geq \frac{1}{S} \sum_{\ell=1}^{S} I\left(X_{\ell} ; \hat{X}_{\ell} \mid X_{1}^{\ell-1}\right)-\frac{1}{S} \sum_{\ell=1}^{S} I\left(X_{\ell} ; U \mid X_{1}^{\ell-1}\right) \\
& \geq \frac{1}{S} \sum_{\ell=1}^{S} I\left(X_{\ell} ; \hat{X}_{\ell} \mid X_{1}^{\ell-1}\right)-\frac{1}{S} C \\
& \geq \frac{1}{S} \sum_{\ell=1}^{S} \mathrm{R}_{X_{\ell} \mid X_{1}^{\ell-1}}\left(D_{\ell}\right)-\frac{1}{S} C \\
& \geq \frac{1}{S}\left(\mathrm{R}_{X_{\mathcal{S}}}\left(D_{\mathcal{S}}\right)-C\right),
\end{aligned}
$$

where (a) follows from the Markov chains in (57), (b) follows from (55), (c) follows from the distortion constraints (56) and the definition of the informational conditional RD function $\mathrm{R}_{X_{\ell} \mid X_{1}^{\ell-1}}\left(D_{\ell}\right)$; and (d) follows from Lemma 33 .

The lower bound for the remaining subsets $\mathcal{S} \subseteq \mathcal{L}$ can be proved by repeating the above arguments with an appropriate relabelling of the variables.

\section{APPENDIX G}

\section{PROOF OF THEOREM 17}

We need the following lemma.

Lemma 34. Take any sequence of $\left(n, \mathcal{M}_{c}^{(n)}, \mathcal{M}^{(n)}\right)$-codes and any positive real sequence $\left\{\alpha_{n}\right\} \downarrow 0$. If for every sufficiently large blocklength $n$ we have

$$
\mathbb{P}\left[\bigcap_{\ell \in \mathcal{L}}\left\{\overline{\mathrm{d}}_{\ell}\left(\hat{X}_{\ell}^{n}, X_{\ell}^{n}\right)<D_{\ell}\right\}\right] \geq 2^{-n \alpha_{n}},
$$

then there exists real sequence $\left\{\zeta_{n}\right\} \rightarrow 0$ such that

$$
\frac{1}{n} \log \left|\mathcal{M}^{(n)}\right| \geq \mathrm{R}\left(\boldsymbol{D}+\zeta_{n}, \frac{1}{n} \log \left|\mathcal{M}_{c}^{(n)}\right|+\zeta_{n}\right)-\zeta_{n} .
$$

Proof: Lemma 34 is proved in Appendix $\mathrm{H}$

Now consider Theorem 17 and any sequence of $\left(n, \mathcal{M}_{\mathrm{c}}^{(n)}, \mathcal{M}^{(n)}\right)$-codes satisfying (16) and (17). Pick a positive real sequence $\left\{\alpha_{n}\right\} \downarrow 0$ satisfying

$$
\lim _{n \rightarrow \infty} 2^{-n \alpha_{n}}=0
$$

Suppose that there exists a large blocklength $n^{*}$ so that for all $n>n^{*}$ :

$$
\mathbb{P}\left[\bigcap_{\ell \in \mathcal{L}}\left\{\overline{\mathrm{d}}_{\ell}\left(\hat{X}_{\ell}^{n}, X_{\ell}^{n}\right)<D_{\ell}\right\}\right] \geq 2^{-n \alpha_{n}}
$$

Pick $\gamma>0$ arbitrarily. By assumptions (16) and (17), and by Lemma 34, we can pick $n^{*}$ sufficiently large so that $\forall n \geq n^{*}$ the following chain of inequalities holds:

$$
\begin{aligned}
\mathrm{R}(\boldsymbol{D}, C)+\gamma & \stackrel{\mathrm{a}}{>} \frac{1}{n} \log \left|\mathcal{M}^{(n)}\right| \\
& \stackrel{\mathrm{b}}{\geq} \mathrm{R}\left(\boldsymbol{D}+\gamma, \frac{1}{n} \log \left|\mathcal{M}_{\mathrm{c}}^{(n)}\right|+\gamma\right)-\gamma \\
& \stackrel{\mathrm{c}}{\geq} \mathrm{R}(\boldsymbol{D}+\gamma, C+2 \gamma)-\gamma,
\end{aligned}
$$

where step (a) follows by assumption (16); step (b) follows from Lemma 34, and step (c) follows by assumption (17) and the fact that the informational RDC function is non-increasing in the cache capacity.

Since the RDC function $\operatorname{R}(\boldsymbol{D}, C)$ is a continuous function of $\boldsymbol{D} \in[0, \infty)^{L}$ and $C \in[0, \infty)$ and by choosing $\gamma$ sufficiently close to 0 , for any desired $\epsilon>0$ we can obtain from 59 that

$$
\begin{aligned}
& \mathrm{R}(\boldsymbol{D}, \boldsymbol{C})-\frac{1}{n} \log \left|\mathcal{M}^{(n)}\right| \\
& \quad \leq \mathrm{R}(\boldsymbol{D}, C)-\mathrm{R}(\boldsymbol{D}+\gamma, C+2 \gamma)+\gamma \\
& \quad<\epsilon
\end{aligned}
$$


This contradicts assumption 16 . We therefore conclude that assumption 58 was wrong and holds with a strict inequality in the reverse direction for some $n \geq n^{*}$ and consequently

$$
\limsup _{n \rightarrow \infty} \mathbb{P}\left[\bigcup_{\ell \in \mathcal{L}}\left\{\overline{\mathrm{d}}_{\ell}\left(\hat{X}_{\ell}^{n}, X_{\ell}^{n}\right) \geq D_{\ell}\right\}\right]=1 \text {. }
$$

\section{APPENDIX H}

PROOF OF LEMMA 34

\section{A. Proof Setup and Outline}

Assume that we have a sequence of $\left(n, \mathcal{M}_{\mathrm{c}}^{(n)}, \mathcal{M}^{(n)}\right)$-codes for the RDC problem. For each blocklength $n$ and RDC code $\left(\phi_{\mathrm{c}}^{(n)}, \phi_{\ell}^{(n)}, \varphi_{\ell}^{(n)}\right)$, let

$$
\left.\mathcal{G}^{(n)}:=\left\{\boldsymbol{x}^{n} \in \boldsymbol{\mathcal { X }}^{n}: \overline{\mathrm{d}}_{\ell}\left(\varphi_{\ell}^{(n)}\left(\mathrm{f}\left(\boldsymbol{x}^{n}\right), \phi_{\mathrm{c}}^{(n)}\left(\boldsymbol{x}^{n}\right)\right), x_{\ell}^{n}\right)\right)<D_{\ell}, \forall \ell \in \mathcal{L}\right\}
$$

denote the set of all "good" sequences that the code will reconstruct with acceptable distortions. Let $\left\{\alpha_{n}\right\} \downarrow 0$ be a sequence of positive real numbers, and suppose that the above mentioned sequence of RDC codes satisfies

$$
\mathbb{P}\left[\boldsymbol{X}^{n} \in \mathcal{G}^{(n)}\right] \geq 2^{-n \alpha_{n}}
$$

for every blocklength $n$. For example, we are free to choose $\left\{\alpha_{n}\right\}$ such that $\left\{2^{-n \alpha_{n}}\right\} \rightarrow 0$ or $\left\{2^{-n \alpha_{n}}\right\} \rightarrow 1$.

The basic idea of the following proof is to show that (62) implies that the delivery-phase rate of the sequence of RDC codes satisfies

$$
\frac{1}{n} \log \left|\mathcal{M}^{(n)}\right| \geq \mathrm{R}\left(\boldsymbol{D}+\zeta_{n}, \frac{1}{n} \log \left|\mathcal{M}_{\mathrm{c}}^{(n)}\right|+\zeta_{n}\right)-\zeta_{n}
$$

for some sequence $\left\{\zeta_{n}\right\} \rightarrow 0$. The key idea in proving this inequality will be to use the RDC code on a hypothetical "perturbed" source that is constructed from the good set $\mathcal{G}^{(n)}$ and the DMS of pmf $p_{\boldsymbol{X}}$.

\section{B. Construction of the Perturbed Source}

The following construction is similar to that used by Watanabe [28] and Gu and Effros [29]. Let us call the DMS

$$
\boldsymbol{X}^{n} \sim p_{\boldsymbol{X}}^{n}\left(\boldsymbol{x}^{n}\right)=\prod_{i=1}^{n} p_{\boldsymbol{X}}\left(\boldsymbol{x}_{i}\right), \quad \boldsymbol{x}^{n} \in \boldsymbol{\mathcal { X }}^{n}
$$

the real source. The perturbed source

$$
\boldsymbol{Y}^{n} \sim q_{\boldsymbol{Y}^{n}}\left(\boldsymbol{y}^{n}\right)=\mathbb{P}\left[\boldsymbol{Y}^{n}=\boldsymbol{y}^{n}\right] \quad \boldsymbol{y}^{n} \in \boldsymbol{\mathcal { X }}^{n}
$$

is defined as follows: If $\boldsymbol{y} \in \mathcal{G}_{n}$, then

$$
q_{\boldsymbol{Y}^{n}}\left(\boldsymbol{y}^{n}\right)=\frac{2^{n\left(\alpha_{n}+\frac{1}{\sqrt{n}}\right)} p_{\boldsymbol{X}}^{n}\left(\boldsymbol{y}^{n}\right)}{2^{n\left(\alpha_{n}+\frac{1}{\sqrt{n}}\right)} \mathbb{P}\left[\boldsymbol{X}^{n} \in \mathcal{G}_{n}\right]+\mathbb{P}\left[\boldsymbol{X}^{n} \notin \mathcal{G}_{n}\right]} .
$$

Otherwise if $\boldsymbol{y}^{n} \notin \mathcal{G}_{n}$, then

$$
q_{\boldsymbol{Y}^{n}}\left(\boldsymbol{y}^{n}\right)=\frac{p_{\boldsymbol{X}}^{n}\left(\boldsymbol{y}^{n}\right)}{2^{n\left(\alpha_{n}+\frac{1}{\sqrt{n}}\right)} \mathbb{P}\left[\boldsymbol{X}^{n} \in \mathcal{G}_{n}\right]+\mathbb{P}\left[\boldsymbol{X}^{n} \notin \mathcal{G}_{n}\right]}
$$

It is worth noting that $q_{\boldsymbol{Y}^{n}}$ need not be a product distribution on $\mathcal{X}^{n}$. It is, however, not too difficult to see that $q_{\boldsymbol{Y}^{n}}$ is "close" to the product distribution $p_{\boldsymbol{X}}^{n}$ of the real DMS in the following sense. For every sequence $\boldsymbol{y}^{n} \in \mathcal{X}^{n}$ :

$$
2^{-n\left(\alpha_{n}+\frac{1}{\sqrt{n}}\right)} p_{\boldsymbol{X}}^{n}\left(\boldsymbol{y}^{n}\right) \leq q_{\boldsymbol{Y}^{n}}\left(\boldsymbol{y}^{n}\right) \leq 2^{n\left(\alpha_{n}+\frac{1}{\sqrt{n}}\right)} p_{\boldsymbol{X}}^{n}\left(\boldsymbol{y}^{n}\right) .
$$




\section{Caching the Perturbed Source - Distortion Bounds}

We now take the $\left(n, \mathcal{M}_{\mathrm{c}}^{(n)}, \mathcal{M}^{(n)}\right)$-code $\left(\phi_{\mathrm{c}}^{(n)}, \phi_{\ell}^{(n)}, \varphi_{\ell}^{(n)}\right)$ from the above mentioned sequence, and use it to cache the perturbed source $\boldsymbol{Y}^{n} \sim q_{\boldsymbol{Y}^{n}}$. For each $\ell \in \mathcal{L}$, let

$$
\hat{Y}_{\ell}^{n}=\varphi_{\ell}^{(n)}\left(\phi_{\mathrm{c}}^{(n)}\left(\boldsymbol{Y}^{n}\right), \phi_{\ell}^{(n)}\left(\boldsymbol{Y}^{n}\right)\right)
$$

denote the corresponding output at the decoder. A lower bound on the probability of the decoding success for this RDC code on $\boldsymbol{Y}^{n}$ can be obtained as follows:

$$
\begin{aligned}
& \mathbb{P}\left[\boldsymbol{Y}^{n} \in \mathcal{G}^{(n)}\right] \\
& =\sum_{\boldsymbol{y}^{n} \in \mathcal{G}^{(n)}} q_{\boldsymbol{Y}^{n}\left(\boldsymbol{y}^{n}\right)} \\
& \stackrel{\mathrm{a}}{=} \sum_{\boldsymbol{y}^{n} \in \mathcal{G}^{(n)}} \frac{2^{n\left(\alpha_{n}+\frac{1}{\sqrt{n}}\right)} p_{\boldsymbol{X}}\left(\boldsymbol{y}^{n}\right)}{2^{n\left(\alpha_{n}+\frac{1}{\sqrt{n}}\right)} \mathbb{P}\left[\boldsymbol{X}^{n} \in \mathcal{G}^{(n)}\right]+1-\mathbb{P}\left[\boldsymbol{X}^{n} \in \mathcal{G}^{(n)}\right]} \\
& =\frac{2^{n\left(\alpha_{n}+\frac{1}{\sqrt{n}}\right)} \mathbb{P}\left[\boldsymbol{X}^{n} \in \mathcal{G}^{(n)}\right]}{2^{n\left(\alpha_{n}+\frac{1}{\sqrt{n}}\right)} \mathbb{P}\left[\boldsymbol{X}^{n} \in \mathcal{G}^{(n)}\right]+1-\mathbb{P}\left[\boldsymbol{X}^{n} \in \mathcal{G}^{(n)}\right]} \\
& =\frac{2^{n\left(\alpha_{n}+\frac{1}{\sqrt{n}}\right)}}{2^{n\left(\alpha_{n}+\frac{1}{\sqrt{n}}\right)}+\frac{1}{\mathbb{P}\left[\boldsymbol{X}^{n} \in \mathcal{G}^{(n)}\right]}-1} \\
& \mathrm{\textrm {b }} \frac{2^{n\left(\alpha_{n}+\frac{1}{\sqrt{n}}\right)}}{2^{n\left(\alpha_{n}+\frac{1}{\sqrt{n}}\right)}+2^{n \alpha_{n}}-1} \\
& =\frac{2^{\sqrt{n}}}{2^{\sqrt{n}}+1-2^{-n \alpha_{n}}} \\
& \geq \frac{2^{\sqrt{n}}}{2^{\sqrt{n}}+1}
\end{aligned}
$$

where (a) substitutes the definition of $q_{\boldsymbol{Y}^{n}}\left(\boldsymbol{y}^{n}\right)$ from 64 and (b) invokes the assumption 62]. Therefore,

$$
\lim _{n \rightarrow \infty} \mathbb{P}\left[\boldsymbol{Y}^{n} \in \mathcal{G}^{(n)}\right]=1 .
$$

The expected distortion performance of the RDC code on $\boldsymbol{Y}^{n} \sim q_{\boldsymbol{Y}^{n}}$ can be upper bounded by

$$
\begin{aligned}
\mathbb{E}\left[\overline{\mathrm{d}}_{\ell}\left(\hat{Y}_{\ell}^{n}, Y_{\ell}^{n}\right)\right]= & \mathbb{E}\left[\overline{\mathrm{d}}_{\ell}\left(\hat{Y}_{\ell}^{n}, Y_{\ell}^{n}\right) \mid \boldsymbol{Y}^{n} \in \mathcal{G}^{(n)}\right] \mathbb{P}\left[\boldsymbol{Y}^{n} \in \mathcal{G}^{(n)}\right] \\
& +\mathbb{E}\left[\overline{\mathrm{d}}_{\ell}\left(\hat{Y}_{\ell}^{n}, Y_{\ell}^{n}\right) \mid \boldsymbol{Y}^{n} \notin \mathcal{G}^{(n)}\right] \mathbb{P}\left[\boldsymbol{Y}^{n} \notin \mathcal{G}^{(n)}\right] \\
& \leq D_{\ell}+D_{\max }\left(1-\frac{2^{\sqrt{n}}}{2^{\sqrt{n}}+1}\right) .
\end{aligned}
$$

Therefore,

$$
\limsup _{n \rightarrow \infty} \mathbb{E}\left[\overline{\mathrm{d}}_{\ell}\left(\hat{Y}_{\ell}^{n}, Y_{\ell}^{n}\right)\right] \leq D_{\ell}, \quad \forall \ell \in \mathcal{L} .
$$

\section{Caching the Perturbed Source - A Lower Bound on the Caching Rate}

We now use a slight modification of the converse proof in Appendix A to give a single-letter lower bound on the caching rate for the perturbed source. Let $M_{\mathrm{c}}^{(n)}=\phi_{\mathrm{c}}^{(n)}\left(\boldsymbol{Y}^{n}\right)$ in $\mathcal{M}_{\mathrm{c}}^{(n)}$ denote the corresponding cache message. We have

$$
\begin{aligned}
& \frac{1}{n} \log \left|\mathcal{M}_{\mathrm{c}}^{(n)}\right| \geq \frac{1}{n} H\left(M_{\mathrm{c}}^{(n)}\right) \geq \frac{1}{n} I\left(\boldsymbol{Y}^{n} ; M_{\mathrm{c}}^{(n)}\right) \\
& =\frac{1}{n} \sum_{i=1}^{n} I\left(\boldsymbol{Y}_{i} ; M_{\mathrm{c}}^{(n)} \mid \boldsymbol{Y}_{1}^{i-1}\right) \\
& \stackrel{\mathrm{a}}{=} \frac{1}{n} \sum_{i=1}^{n} I\left(\boldsymbol{Y}_{i} ; M_{\mathrm{c}}^{(n)}, \boldsymbol{Y}_{1}^{i-1}\right)-I\left(\boldsymbol{Y}_{i} ; \boldsymbol{Y}_{1}^{i-1}\right) \\
& \stackrel{\mathrm{b}}{=} \frac{1}{n} \sum_{i=1}^{n} I\left(\boldsymbol{Y}_{i} ; U_{i}\right)-\frac{1}{n} \sum_{i=1}^{n} H\left(\boldsymbol{Y}_{i}\right)+\frac{1}{n} \sum_{i=1}^{n} H\left(\boldsymbol{Y}_{i} \mid \boldsymbol{Y}_{1}^{i-1}\right) \\
& =\frac{1}{n} \sum_{i=1}^{n} I\left(\boldsymbol{Y}_{i} ; U_{i}\right)-\frac{1}{n} \sum_{i=1}^{n} H\left(\boldsymbol{Y}_{i}\right)+\frac{1}{n} H\left(\boldsymbol{Y}^{n}\right),
\end{aligned}
$$


where in (a) we note that $q_{Y^{n}}$ need not be a product measure and (b) substitutes

$$
U_{i}=\left(M_{\mathrm{c}}^{(n)}, \boldsymbol{Y}_{1}^{i-1}\right) \quad \text { on } \quad \mathcal{U}_{i}=\mathcal{M}_{\mathrm{c}}^{(n)} \times \mathcal{X}^{i-1}
$$

in the same way as the weak converse in Appendix A.

\section{E. Caching the Perturbed Source - A Lower Bound on the Delivery Rate}

Now consider an arbitrary request $\ell \in \mathcal{L}$, and let $M_{\ell}^{(n)}=\phi_{\ell}^{(n)}\left(\boldsymbol{Y}^{n}\right)$ in $\mathcal{M}^{(n)}$ denote the corresponding delivery phase message. The delivery-phase rate can be lower bound as follows:

$$
\begin{aligned}
\frac{1}{n} \log \left|\mathcal{M}^{(n)}\right| & \geq \frac{1}{n} H\left(M_{\ell}^{(n)} \mid M_{\mathrm{c}}^{(n)}\right) \\
& \geq \frac{1}{n} I\left(\boldsymbol{Y}^{n} ; M_{\ell}^{(n)} \mid M_{\mathrm{c}}^{(n)}\right) \\
& \geq \frac{1}{n} I\left(\boldsymbol{Y}^{n} ; \hat{Y}_{\ell}^{n} \mid M_{\mathrm{c}}^{(n)}\right) \\
& =\frac{1}{n} \sum_{i=1}^{n} I\left(\boldsymbol{Y}_{i} ; \hat{Y}_{\ell}^{n} \mid M_{\mathrm{c}}^{(n)}, \boldsymbol{Y}_{1}^{i-1}\right) \\
& \geq \frac{1}{n} \sum_{i=1}^{n} I\left(Y_{\ell, i} ; \hat{Y}_{\ell, i} \mid U_{i}\right)
\end{aligned}
$$

where (a) follows because $\hat{Y}_{\ell}^{n} \leftrightarrow\left(M_{\ell}^{(n)}, M_{\mathrm{c}}^{(n)}\right) \leftrightarrow \boldsymbol{Y}^{n}$ forms a Markov chain; and (b) substitutes $U_{i}$ as in the proof of the weak converse in Appendix A.

\section{F. Caching the Perturbed Source - Timesharing and Cardinality Reduction}

Consider the tuple of random variables $\left(\boldsymbol{Y}^{n}, U^{n}, \hat{\boldsymbol{Y}}^{n}\right)$ constructed in the above sections. Let $J \in\{1,2, \ldots, n\}$ be a uniform random variable that is independent of $\left(\boldsymbol{Y}^{n}, U^{n}, \hat{\boldsymbol{Y}}^{n}\right)$, and let

$$
\overline{\mathcal{U}}^{(n)}=\left(\bigcup_{i=1}^{n} \mathcal{U}_{i}\right) \times\{1,2, \ldots, n\} .
$$

Let $(\overline{\boldsymbol{Y}}, \bar{U}, \hat{\overline{\boldsymbol{Y}}}) \in \mathcal{X} \times \overline{\mathcal{U}} \times \hat{\mathcal{X}}$, denote the random tuples generated by setting

$$
\overline{\boldsymbol{Y}}=\boldsymbol{Y}_{J}, \quad \bar{U}=\left(U_{J}, J\right) \text { and } \hat{\overline{\boldsymbol{Y}}}=\hat{\boldsymbol{Y}}_{J}
$$

With this choice, it then follows from 67 that

$$
\begin{aligned}
\frac{1}{n} \log \left|\mathcal{M}_{\mathrm{c}}^{(n)}\right| & \geq I\left(\boldsymbol{Y}_{J} ; U_{J} \mid J\right)-H\left(\boldsymbol{Y}_{J}\right)+\frac{1}{n} H\left(\boldsymbol{Y}^{n}\right) \\
& =I(\overline{\boldsymbol{Y}} ; \bar{U})-H(\overline{\boldsymbol{Y}})+\frac{1}{n} H\left(\boldsymbol{Y}^{n}\right)
\end{aligned}
$$

and from 68 that

$$
\begin{aligned}
\frac{1}{n} \log \left|\mathcal{M}^{(n)}\right| & \geq I\left(Y_{\ell, J} ; \hat{Y}_{\ell, J} \mid U_{J}, J\right) \\
& =I\left(\bar{Y}_{\ell} ; \hat{\bar{Y}}_{\ell} \mid \bar{U}\right) .
\end{aligned}
$$

Finally, from 66 the expected distortion for satisfies

$$
\begin{aligned}
\mathbb{E}\left[\mathrm{d}_{\ell}\left(\hat{\bar{Y}}_{\ell}, \bar{Y}_{\ell}\right)\right] & =\mathbb{E}\left[\overline{\mathrm{d}}\left(\hat{Y}_{\ell}^{n}, Y_{\ell}^{n}\right)\right] \\
& \leq D_{\ell}+D_{\max }\left(1-\frac{2^{\sqrt{n}}}{2^{\sqrt{n}}+1}\right)
\end{aligned}
$$

Let $q_{\overline{\boldsymbol{Y}} \bar{U} \hat{\overline{\boldsymbol{Y}}}}$ denote the joint distribution of the variables $(\overline{\boldsymbol{Y}}, \bar{U}, \hat{\overline{\boldsymbol{Y}}})$. The cardinality of $\overline{\mathcal{U}}^{(n)}$ grows without bound in $n$, and the next lemma uses the convex cover method [22, Appendix C] (see also the arguments in A to bound this cardinality by a finite number.

Lemma 35. There exists a random tuple $(\overline{\overline{\boldsymbol{Y}}}, \overline{\bar{U}}, \hat{\overline{\boldsymbol{Y}}}) \sim q_{\overline{\overline{\boldsymbol{Y}}} \overline{\bar{U}}}$, defined on $\mathcal{\mathcal { X }} \times \hat{\overline{\mathcal{U}}} \times \hat{\mathcal{X}}$ for which the following is true:

- $|\overline{\bar{U}}| \leq|\mathcal{X}|+2 L$, 
- $q_{\overline{\mathbf{Y}}}=q_{\overline{\mathbf{Y}}}$,

- $I(\overline{\overline{\boldsymbol{Y}}} ; \overline{\bar{U}})=I(\overline{\boldsymbol{Y}} ; \bar{U})$,

- $I\left(\overline{\bar{Y}}_{\ell} ; \hat{\bar{Y}}_{\ell} \mid \overline{\bar{U}}\right)=I\left(\bar{Y}_{\ell} ; \hat{\bar{Y}}_{\ell} \mid \bar{U}\right)$ for all $\ell \in \mathcal{L}$, and

- $\mathbb{E}\left[\mathrm{d}_{\ell}\left(\hat{\bar{Y}}_{\ell}, \overline{\bar{Y}}_{\ell}\right)\right]=\mathbb{E}\left[\mathrm{d}_{\ell}\left(\hat{\bar{Y}}_{\ell}, \bar{Y}_{\ell}\right)\right]$ for all $\ell \in \mathcal{L}$.

Combining Lemma 35 with 69, (70) and 771 yields the following: There exists some tuple

$$
(\overline{\overline{\boldsymbol{Y}}}, \overline{\bar{U}}, \hat{\overline{\boldsymbol{Y}}}) \sim q_{\overline{\overline{\boldsymbol{Y}}} \overline{\bar{U}} \hat{\overline{\boldsymbol{Y}}}} \quad \text { on } \quad \boldsymbol{\mathcal { X }} \times \overline{\overline{\mathcal{U}}} \times \hat{\mathcal{X}}
$$

such that cache rate is lower bounded by

$$
\frac{1}{n} \log \left|\mathcal{M}_{\mathrm{c}}^{(n)}\right| \geq I(\overline{\overline{\boldsymbol{Y}}} ; \overline{\bar{U}})-H(\overline{\overline{\boldsymbol{Y}}})+\frac{1}{n} H\left(\boldsymbol{Y}^{n}\right) ;
$$

the expected distortion is upper bounded by

$$
\mathbb{E}\left[\mathrm{d}_{\ell}\left(\hat{\bar{Y}}_{\ell}, \overline{\bar{Y}}_{\ell}\right)\right] \leq D_{\ell}+D_{\max }\left(1-\frac{2^{\sqrt{n}}}{2^{\sqrt{n}}+1}\right) ;
$$

and the delivery phase rate is lower bounded by

$$
\begin{aligned}
& \frac{1}{n} \log \left|\mathcal{M}^{(n)}\right| \geq I\left(\overline{\bar{Y}}_{\ell} ; \hat{\overline{\bar{Y}}}_{\ell} \mid \overline{\bar{U}}\right) \\
& \geq \mathrm{R}_{\bar{Y}_{\ell} \mid \overline{\bar{U}}}\left(D_{\ell}+D_{\max }\left(1-\frac{2^{\sqrt{n}}}{2^{\sqrt{n}}+1}\right)\right),
\end{aligned}
$$

where the second inequality follows from the definition of the conditional RD function.

\section{G. Convergence of $H(\overline{\overline{\boldsymbol{Y}}})$ to $H(\boldsymbol{X})$}

Fix $\gamma>0$ arbitrarily small. The set of $\gamma$-letter typical sequences [24] with respect to the DMS $p_{\boldsymbol{X}}^{n}$ will be useful in the following arguments. This set is given by

$$
\mathcal{A}_{\gamma}^{(n)}\left(p_{\boldsymbol{X}}^{n}\right)=\left\{\boldsymbol{x}^{n} \in \mathcal{X}^{n}:\left|\frac{1}{n} \mathrm{~N}\left(\boldsymbol{a} \mid \boldsymbol{x}^{n}\right)-p_{\boldsymbol{X}}(\boldsymbol{a})\right| \leq \gamma p_{\boldsymbol{X}}(\boldsymbol{a}), \forall \boldsymbol{a} \in \mathcal{X}\right\} .
$$

Lemma 36. The probability that the real DMS $\boldsymbol{X}^{n} \sim p_{\boldsymbol{X}}$ does not emit an $\gamma$-letter typical sequence satisfies [24. Thm. 1.1]

$$
\mathbb{P}\left[\boldsymbol{X}^{n} \notin \mathcal{A}_{\gamma}^{(n)}\left(p_{\boldsymbol{X}}\right)\right] \leq 2|\mathcal{X}| 2^{-n \gamma^{2} \mu\left(p_{\boldsymbol{X}}\right)},
$$

where $\mu\left(p_{\boldsymbol{X}}\right)$ is the smallest value of $p_{\boldsymbol{X}}$ on its support $\operatorname{set} \operatorname{supp}\left(p_{\boldsymbol{X}}\right)$.

Let us now return to the perturbed source $\boldsymbol{Y}^{n} \sim q_{\boldsymbol{Y}^{n}}$. For each $\boldsymbol{a} \in \mathcal{X}$ we have

$$
\begin{aligned}
& q_{\overline{\overline{\boldsymbol{Y}}}}(\boldsymbol{a}) \\
& \stackrel{\mathrm{a}}{=} q_{\overline{\boldsymbol{Y}}}(\boldsymbol{a}) \\
& \stackrel{\mathrm{b}}{=} \sum_{\boldsymbol{y}^{n} \in \mathcal{X}^{n}} q_{\boldsymbol{Y}^{n}}\left(\boldsymbol{y}^{n}\right) \mathbb{P}\left[\overline{\boldsymbol{Y}}=\boldsymbol{a} \mid \boldsymbol{Y}^{n}=\boldsymbol{y}^{n}\right] \\
& \stackrel{\mathrm{c}}{=} \sum_{\boldsymbol{y}^{n} \in \mathcal{X}^{n}} q_{\boldsymbol{Y}^{n}}\left(\boldsymbol{y}^{n}\right) \frac{\mathrm{N}\left(\boldsymbol{a} \mid \boldsymbol{y}^{n}\right)}{n} \\
& =\sum_{\boldsymbol{y}^{n} \in \mathcal{A}_{\gamma}^{(n)}} q_{\boldsymbol{Y}^{n}}\left(\boldsymbol{y}^{n}\right) \frac{N\left(\boldsymbol{a} \mid \boldsymbol{y}^{n}\right)}{n}+\sum_{\boldsymbol{y}^{n} \notin \mathcal{A}_{\gamma}^{(n)}} q_{\boldsymbol{Y}^{n}}\left(\boldsymbol{y}^{n}\right) \frac{N\left(\boldsymbol{a} \mid \boldsymbol{y}^{n}\right)}{n} \\
& \stackrel{\mathrm{d}}{\leq} p_{\boldsymbol{X}}(\boldsymbol{a})(1+\gamma) \mathbb{P}\left[\boldsymbol{X}^{n} \in \mathcal{A}_{\gamma}^{(n)}\right]+\mathbb{P}\left[\boldsymbol{X}^{n} \notin \mathcal{A}_{\gamma}^{(n)}\right] \\
& \stackrel{\mathrm{e}}{\leq} p_{\boldsymbol{X}}(\boldsymbol{a})(1+\gamma)+2|\mathcal{X}| 2^{-n \gamma^{2} \mu\left(p_{\boldsymbol{X}}\right)}
\end{aligned}
$$

where (a) applies Lemma 35. (b) and (c) use the fact that $\overline{\boldsymbol{Y}}$ is generated by uniformly at random selecting symbols from $Y^{n}$ (the timesharing argument above); (d) uses the definition of $\gamma$-letter typical sequences; and (e) invokes Lemma 36 . Using similar arguments, we obtain

$$
q_{\overline{\boldsymbol{Y}}}(\boldsymbol{a}) \geq p_{\boldsymbol{X}}(\boldsymbol{a})(1-\gamma)\left(1-2^{-n \gamma^{2} \mu\left(p_{\boldsymbol{X}}\right)}\right) .
$$


From (75) and 276, we have

$$
(1-\gamma) p_{\boldsymbol{X}}(\boldsymbol{a}) \leq \liminf _{n \rightarrow \infty} q_{\overline{\bar{Y}}}(\boldsymbol{a}) \leq \limsup _{n \rightarrow \infty} q_{\overline{\bar{Y}}}(\boldsymbol{a}) \leq(1+\gamma) p_{\boldsymbol{X}}(\boldsymbol{a})
$$

Since (77) holds for every $\gamma>0$, and the sequence $\left\{q_{\overline{\boldsymbol{Y}}}\right\}$ does not dependent on $\gamma$, we have

$$
\lim _{n \rightarrow \infty} q_{\overline{\mathbf{Y}}}(\boldsymbol{a})=p_{\boldsymbol{X}}(\boldsymbol{a}), \quad \forall \boldsymbol{a} \in \mathcal{X} .
$$

Therefore, by the continuity of entropy [30, Chap. 2.3] we have

$$
\lim _{n \rightarrow \infty} H(\overline{\bar{Y}})=H(\boldsymbol{X})
$$

\section{H. Convergence of $(1 / n) H\left(\boldsymbol{Y}^{n}\right)$ to $H(\boldsymbol{X})$}

It follows from 65 that for all $\boldsymbol{a}^{n} \in \mathcal{X}^{n}$ we have

$$
\begin{array}{r}
-\alpha_{n}-\frac{1}{\sqrt{n}} \leq \frac{1}{n} \log p_{\boldsymbol{X}}^{n}\left(\boldsymbol{a}^{n}\right)-\frac{1}{n} \log q_{\boldsymbol{Y}^{n}}\left(\boldsymbol{a}^{n}\right) \\
\leq \alpha_{n}+\frac{1}{\sqrt{n}}
\end{array}
$$

Moreover, for every $\boldsymbol{a}^{n} \in \mathcal{A}_{\gamma}^{(n)}\left(p_{\boldsymbol{X}}\right)$ we have

$$
\begin{aligned}
\frac{1}{n} \log \frac{1}{p_{\boldsymbol{X}}^{n}\left(\boldsymbol{a}^{n}\right)} & \stackrel{\mathrm{a}}{=} \frac{1}{n} \log \left(\prod_{i=1}^{n} \frac{1}{p_{\boldsymbol{X}}\left(\boldsymbol{a}_{i}\right)}\right) \\
& =\frac{1}{n} \sum_{i=1}^{n} \log \frac{1}{p_{\boldsymbol{X}}\left(\boldsymbol{a}_{i}\right)} \\
& =\frac{1}{n} \sum_{\boldsymbol{a}^{\prime} \in \mathcal{X}} \mathrm{N}\left(\boldsymbol{a}^{\prime} \mid \boldsymbol{a}^{n}\right) \log \frac{1}{p_{\boldsymbol{X}}\left(\boldsymbol{a}^{\prime}\right)} \\
& \leq(1+\gamma) \sum_{\boldsymbol{a}^{\prime} \in \mathcal{X}} p_{\boldsymbol{X}}\left(\boldsymbol{a}^{\prime}\right) \log \frac{1}{p_{\boldsymbol{X}}\left(\boldsymbol{a}^{\prime}\right)} \\
& =(1+\gamma) H(\boldsymbol{X})
\end{aligned}
$$

where (a) follows because $p_{\boldsymbol{X}}^{n}$ is a product measure and (b) follows because $\boldsymbol{a}^{n} \in \mathcal{A}_{\gamma}^{(n)}\left(p_{\boldsymbol{X}}\right)$. Similarly, we have

$$
\frac{1}{n} \log \frac{1}{p_{\boldsymbol{X}}^{n}\left(\boldsymbol{a}^{n}\right)} \geq(1-\gamma) H(\boldsymbol{X})
$$

for all $\boldsymbol{a}^{n} \in \mathcal{X}^{n}$.

Now consider the joint entropy $H\left(\boldsymbol{Y}^{n}\right)$. With a few manipulations, we obtain the upper bound in (84). Here step (a) uses $(80)$. Step (b) uses the upper bound in 82 on the first logarithmic term, and

$$
\begin{aligned}
\frac{1}{n} \log \frac{1}{p_{\boldsymbol{X}}^{n}\left(\boldsymbol{a}^{n}\right)} & =\frac{1}{n} \sum_{i=1}^{n} \log \frac{1}{p_{\boldsymbol{X}}\left(\boldsymbol{a}_{i}\right)} \\
& \leq \frac{1}{n} \sum_{i=1}^{n} \log \frac{1}{\mu\left(p_{\boldsymbol{X}}\right)} \\
& =\log \frac{1}{\mu\left(p_{\boldsymbol{X}}\right)}
\end{aligned}
$$

on the second term $4^{4}$ Finally, step (c) applies Lemma 36 Using similar arguments, we also have

$$
\begin{aligned}
& \frac{1}{n} H\left(\boldsymbol{Y}^{n}\right) \\
& =\frac{1}{n} \sum_{\boldsymbol{a}^{n} \in \operatorname{supp}\left(q_{\left.\boldsymbol{Y}^{n}\right)}\right.} q_{\boldsymbol{Y}}^{n}\left(\boldsymbol{a}^{n}\right) \log \frac{1}{q_{\boldsymbol{Y}}\left(\boldsymbol{a}^{n}\right)} \\
& \geq \sum_{\boldsymbol{a}^{n} \in \mathcal{A}_{\gamma}^{(n)}\left(p_{\boldsymbol{X}}\right)} q_{\boldsymbol{Y}}^{n}\left(\boldsymbol{a}^{n}\right)\left(\frac{1}{n} \log \frac{1}{p_{\boldsymbol{X}}^{n}\left(\boldsymbol{a}^{n}\right)}-\alpha_{n}-\frac{1}{\sqrt{n}}\right)
\end{aligned}
$$

${ }^{4}$ If $p_{\boldsymbol{X}}^{n}\left(\boldsymbol{a}^{n}\right)=0$, then by definition $q_{\boldsymbol{Y}^{n}}\left(\boldsymbol{a}^{n}\right)=0$ and $\boldsymbol{a}^{n} \notin \operatorname{supp}\left(q_{\boldsymbol{Y}^{n}}\right)$. 


$$
\begin{aligned}
& \frac{1}{n} H\left(\boldsymbol{Y}^{n}\right)=\frac{1}{n} \sum_{\boldsymbol{a}^{n} \in \operatorname{supp}\left(q_{\left.\boldsymbol{Y}^{n}\right)}\right.} q_{\boldsymbol{Y}^{n}}\left(\boldsymbol{a}^{n}\right) \log \frac{1}{q_{\boldsymbol{Y}}\left(\boldsymbol{a}^{n}\right)} \\
& \stackrel{\mathrm{a}}{\leq} \sum_{\boldsymbol{a}^{n} \in \operatorname{supp}\left(q_{\left.\boldsymbol{Y}^{n}\right)}\right.} q_{\boldsymbol{Y}^{n}}\left(\boldsymbol{a}^{n}\right)\left(\frac{1}{n} \log \frac{1}{p_{\boldsymbol{X}}^{n}\left(\boldsymbol{a}^{n}\right)}+\alpha_{n}+\frac{1}{\sqrt{n}}\right) \\
& =\sum_{\boldsymbol{a}^{n} \in \mathcal{A}_{\gamma}^{(n)}\left(p_{\boldsymbol{X}}\right) \cap \operatorname{supp}\left(q_{\boldsymbol{Y}^{n}}\right)} q_{\boldsymbol{Y}^{n}}\left(\boldsymbol{a}^{n}\right)\left(\frac{1}{n} \log \frac{1}{p_{\boldsymbol{X}}^{n}\left(\boldsymbol{a}^{n}\right)}+\alpha_{n}+\frac{1}{\sqrt{n}}\right) \\
& +\sum_{\boldsymbol{a}^{n} \notin \mathcal{A}_{\gamma}^{(n)}\left(p_{\boldsymbol{X}}\right) \cap \operatorname{supp}\left(q_{\boldsymbol{Y}^{n}}\right)} q_{\boldsymbol{Y}^{n}}\left(\boldsymbol{a}^{n}\right)\left(\frac{1}{n} \log \frac{1}{p_{\boldsymbol{X}}^{n}\left(\boldsymbol{a}^{n}\right)}+\alpha_{n}+\frac{1}{\sqrt{n}}\right) \\
& \stackrel{\mathrm{b}}{\leq} \sum_{\boldsymbol{a}^{n} \in \mathcal{A}_{\gamma}^{(n)}\left(p_{\mathbf{X}}\right) \cap \operatorname{supp}\left(q_{\boldsymbol{Y}^{n}}\right)} q_{\boldsymbol{Y}^{n}}\left(\boldsymbol{a}^{n}\right)\left((1+\gamma) H(\boldsymbol{X})+\alpha_{n}+\frac{1}{\sqrt{n}}\right) \\
& +\sum_{\boldsymbol{a}^{n} \notin \mathcal{A}_{\gamma}^{(n)}\left(p_{\boldsymbol{X}}\right) \cap \operatorname{supp}\left(q_{\boldsymbol{Y}^{n}}\right)} q_{\boldsymbol{Y}^{n}}\left(\boldsymbol{a}^{n}\right)\left(\log \frac{1}{\mu\left(p_{\boldsymbol{X}}\right)}+\alpha_{n}+\frac{1}{\sqrt{n}}\right) \\
& \stackrel{\mathrm{c}}{\leq}(1+\gamma) H(\boldsymbol{X})+\alpha_{n}+\frac{1}{\sqrt{n}}+2|\boldsymbol{X}| 2^{-n \gamma \mu\left(p_{\boldsymbol{X}}\right)}\left(\log \frac{1}{\mu\left(p_{\boldsymbol{X}}\right)}+\alpha_{n}+\frac{1}{\sqrt{n}}\right)
\end{aligned}
$$

$$
\begin{aligned}
& \stackrel{\mathrm{b}}{\geq} \sum_{\boldsymbol{a}^{n} \in \mathcal{A}_{\gamma}^{(n)}\left(p_{\boldsymbol{X}}\right)} q_{\boldsymbol{Y}}^{n}\left(\boldsymbol{a}^{n}\right)\left((1-\gamma) H(\boldsymbol{X})-\alpha_{n}-\frac{1}{\sqrt{n}}\right) \\
& \stackrel{\mathrm{c}}{\geq}\left((1-\gamma) H(\boldsymbol{X})-\alpha_{n}-\frac{1}{\sqrt{n}}\right)\left(1-2|\boldsymbol{X}| 2^{-n \gamma \mu\left(p_{\boldsymbol{X}}\right)}\right) .
\end{aligned}
$$

Step (a) follows from [80; ; step (b) follows from (83); and step (c) applies Lemma 36 From 84] and 85] we have for every fixed $\gamma>0$

$$
(1-\gamma) H(\boldsymbol{X}) \leq \liminf _{n \rightarrow \infty} \frac{1}{n} H\left(\boldsymbol{Y}^{n}\right) \leq \limsup _{n \rightarrow \infty} \frac{1}{n} H\left(\boldsymbol{Y}^{n}\right) \leq(1+\gamma) H(\boldsymbol{X}),
$$

which, in turn, implies

$$
\lim _{n \rightarrow \infty} \frac{1}{n} H\left(\boldsymbol{Y}^{n}\right)=H(\boldsymbol{X})
$$

\section{Completing the Proof}

The above arguments show that there exists a sequence of random variables 5

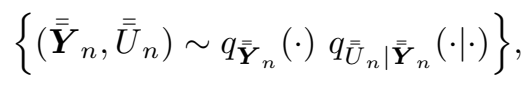

with each $\left(\overline{\bar{Y}}_{n}, \overline{\bar{U}}_{n}\right)$ defined on $\mathcal{X} \times \mathcal{U}$, such that

$$
\lim _{n \rightarrow \infty} q_{\overline{\boldsymbol{Y}}_{n}}(\boldsymbol{a})=p_{\boldsymbol{X}}(\boldsymbol{a}), \quad \forall \boldsymbol{a} \in \mathcal{X}
$$

and

$$
\begin{aligned}
& \frac{1}{n} \log \left|\mathcal{M}_{\mathrm{c}}^{(n)}\right| \geq I(\overline{\bar{Y}} ; \overline{\bar{U}})-\epsilon_{1, n} \\
& \frac{1}{n} \log \left|\mathcal{M}_{\mathrm{c}}^{(n)}\right| \geq \mathrm{R}_{\overline{\bar{Y}}_{\ell, n} \mid \overline{\bar{U}}_{n}}\left(D_{\ell}+\epsilon_{2, n}\right), \quad \forall \ell \in \mathcal{L},
\end{aligned}
$$

where

$$
\begin{aligned}
& \epsilon_{1, n}=\left|\frac{1}{n} H\left(\boldsymbol{Y}^{n}\right)-H(\overline{\bar{Y}})\right| \\
& \epsilon_{2, n}=D_{\max }\left(1-\frac{2^{\sqrt{n}}}{2^{\sqrt{n}}-1}\right) .
\end{aligned}
$$

\footnotetext{
${ }^{5}$ Here, for clarity, we have added the subscript $n$ on the random variables to identify the corresponding blocklength $n$.
} 
Let $\left(\boldsymbol{X}, \overline{\bar{U}}_{n}\right) \sim p_{\boldsymbol{X}}(\cdot) q_{\bar{U}_{n} \mid \overline{\boldsymbol{Y}}_{n}}(\cdot \mid \cdot)$, and define

$$
\epsilon_{3, n}=\left|\mathrm{R}_{\overline{\mathbf{Y}}_{n} \mid \overline{\bar{U}}_{n}}\left(D_{\ell}+\epsilon_{2, n}\right)-\mathrm{R}_{\boldsymbol{X} \mid \overline{\bar{U}}_{n}}\left(D_{\ell}+\epsilon_{2, n}\right)\right| .
$$

Finally, choose $\zeta_{n}=\max \left\{\epsilon_{1, n}, \epsilon_{2, n}, \epsilon_{2, n}\right\}$ so that the lemma follows from [78, 797 and 86 and the continuity of the informational conditional RD function.

\section{APPENDIX I}

\section{PROOF OF THEOREM 25}

The proof of Theorem 25 will bootstrap the achievability part of Theorem 3 and the strong converse in Theorem 17

Take the single-symbol distortion functions $\mathbf{d}^{*}$ from $(24)$, and consider $\mathrm{R}_{\mathbf{d}^{*}}^{\dagger}(\boldsymbol{D}, C)$ and $\mathrm{R}_{\mathbf{d}^{*}}^{\ddagger}(\boldsymbol{D}, C)-$ the respective operational RDC functions in the expected and excess distortion settings w.r.t. the separable distortion functions

$$
\overline{\mathbf{d}}^{*}=\left(\overline{\mathrm{d}}_{1}^{*}, \ldots, \overline{\mathrm{d}}_{L}^{*}\right)
$$

where

$$
\overline{\mathrm{d}}_{\ell}^{*}\left(\hat{x}_{\ell}^{n}, x_{\ell}^{n}\right)=\frac{1}{n} \sum_{i=1}^{n} \mathrm{~d}_{\ell}^{*}\left(\hat{x}_{\ell, i}, x_{\ell, i}\right)=\frac{1}{n} \sum_{i=1}^{n} \mathrm{f}_{\ell}\left(\mathrm{d}_{\ell}\left(\hat{x}_{\ell, i}, x_{\ell, i}\right)\right)
$$

\section{Lemma 37.}

$$
\mathrm{R}_{\mathbf{d}^{*}}^{\dagger}(\boldsymbol{D}, C)=\mathrm{R}_{\mathbf{d}^{*}}^{\ddagger}(\boldsymbol{D}, C)=\mathrm{R}_{\mathbf{d}^{*}}(\boldsymbol{D}, C) .
$$

Proof: Apply Theorem 3 with $\overline{\mathbf{d}}^{*}$.

\section{Lemma 38.}

$$
\mathbf{R}_{\mathbf{f}}^{\ddagger}(\boldsymbol{D}, C)=\mathbf{R}_{\mathbf{d}^{*}}^{\ddagger}(\mathbf{f}(\boldsymbol{D}), C) .
$$

Proof: For every $\left(n, \mathcal{M}_{\mathrm{c}}^{(n)}, \mathcal{M}^{(n)}\right)$-code we have

$$
\begin{aligned}
& \mathbb{P}\left[\bigcup_{\ell \in \mathcal{L}}\left\{\overline{\mathrm{fd}}_{\ell}\left(\hat{X}_{\ell}^{n}, X_{\ell}^{n}\right) \geq D_{\ell}\right\}\right] \\
& \stackrel{\mathrm{a}}{=} \mathbb{P}\left[\bigcup_{\ell \in \mathcal{L}}\left\{\mathrm{f}_{\ell}{ }^{-1}\left(\frac{1}{n} \sum_{i=1}^{n} \mathrm{f}_{\ell}\left(\mathrm{d}_{\ell}\left(\hat{X}_{\ell}^{n}, X_{\ell}^{n}\right)\right) \geq D_{\ell}\right\}\right]\right. \\
& =\mathbb{P}\left[\bigcup_{\ell \in \mathcal{L}}\left\{\frac{1}{n} \sum_{i=1}^{n} \mathrm{~d}_{\ell}^{*}\left(\hat{X}_{\ell}^{n}, X_{\ell}^{n}\right) \geq \mathrm{f}_{\ell}\left(D_{\ell}\right)\right\}\right] \\
& \stackrel{\mathrm{b}}{=} \mathbb{P}\left[\bigcup_{\ell \in \mathcal{L}}\left\{\overline{\mathrm{d}}_{\ell}^{*}\left(\hat{X}_{\ell}^{n}, X_{\ell}^{n}\right) \geq \mathrm{f}_{\ell}\left(D_{\ell}\right)\right\}\right] .
\end{aligned}
$$

The left hand side of (a) corresponds to the excess-distortion event for $\mathrm{R}_{\mathbf{f}}^{\ddagger}(\boldsymbol{D}, C)$, and the right hand side of (b) corresponds to the excess-distortion event for $\mathrm{R}_{\mathbf{d}^{*}}^{\ddagger}(\mathbf{f}(\boldsymbol{D}), C)$. Therefore, a sequence of $\left(n, \mathcal{M}_{\mathrm{c}}^{(n)}, \mathcal{M}^{(n)}\right)$-codes can achieve vanishing error probabilities w.r.t. the $\mathbf{f}$-separable distortion functions $\overline{\mathbf{d}}_{\mathbf{f}}$ if and only if it achieves vanishing error probabilities w.r.t. the separable distortion functions $\overline{\mathbf{d}}^{*}$.

\section{Lemma 39.}

$$
\tilde{\mathrm{R}}_{\mathbf{f}, \max -\operatorname{exc}}^{\dagger}(\boldsymbol{D}, C) \leq \mathrm{R}_{\mathbf{f}}^{\ddagger}(\boldsymbol{D}, C) .
$$

Proof: Recall Definition 4 and fix the distortion tuple $\boldsymbol{D}$ and cache capacity $C$. If $R>\mathrm{R}_{\mathbf{f}}^{\ddagger}(\boldsymbol{D}, C)$ then there exists a sequence of $\left(n, \mathcal{M}_{\mathrm{c}}^{(n)}, \mathcal{M}^{(n)}\right)$-codes satisfying (5a), 5b) and (6). For this sequence of codes, let

$$
\mathcal{G}_{n}=\bigcap_{\ell \in \mathcal{L}}\left\{\overline{\mathrm{d}}_{\mathrm{f}_{\ell}}\left(\hat{X}_{\ell}^{n}, X_{\ell}^{n}\right)<D_{\ell}\right\}
$$

and let $\mathcal{G}_{n}^{c}$ denote the complement of $\mathcal{G}_{n}$. Then

$$
\begin{aligned}
& \mathbb{E}\left[\max _{\ell \in \mathcal{L}}\left(\overline{\mathrm{fd}}_{\ell}\left(\hat{X}_{\ell}^{n}, X_{\ell}^{n}\right)-D_{\ell}\right)\right] \\
& =\mathbb{E}\left[\max _{\ell \in \mathcal{L}}\left(\overline{\mathrm{fd}}_{\ell}\left(\hat{X}_{\ell}^{n}, X_{\ell}^{n}\right)-D_{\ell}\right) \mid \mathcal{G}_{n}\right] \mathbb{P}\left[\mathcal{G}_{n}\right] \\
& \quad+\mathbb{E}\left[\max _{\ell \in \mathcal{L}}\left(\overline{\mathrm{fd}}_{\ell}\left(\hat{X}_{\ell}^{n}, X_{\ell}^{n}\right)-D_{\ell}\right) \mid \mathcal{G}_{n}^{c}\right] \mathbb{P}\left[\mathcal{G}_{n}^{c}\right] \\
& \leq D_{\max } \mathbb{P}\left[\mathcal{G}_{n}^{c}\right] .
\end{aligned}
$$


Since $D_{\max }$ is finite and $\mathbb{P}\left[\mathcal{G}_{n}^{c}\right] \rightarrow 0$ by $[6$, we have

$$
\limsup _{n \rightarrow \infty} \mathbb{E}\left[\max _{\ell \in \mathcal{L}}\left(\overline{\mathrm{fd}}_{\ell}\left(\hat{X}_{\ell}^{n}, X_{\ell}^{n}\right)-D_{\ell}\right)\right] \leq 0
$$

and $R \geq \tilde{\mathrm{R}}_{\mathbf{f}, \max -\mathrm{exc}}^{\dagger}(\boldsymbol{D}, C)$ by Definition 9

\section{Lemma 40.}

$$
\tilde{\mathrm{R}}_{\mathbf{f}, \text { max-exc }}^{\dagger}(\boldsymbol{D}, C) \geq \mathbf{R}_{\mathbf{d}^{*}}(\mathbf{f}(\boldsymbol{D}), C) .
$$

Proof: If $\mathrm{R}_{\mathbf{d}^{*}}\left(\boldsymbol{D}^{*}, C\right)=0$, then the lemma immediately follows because we always have $\tilde{\mathrm{R}}_{\mathbf{f}, \text { max-exc }}^{\dagger}(\boldsymbol{D}, C) \geq 0$. We henceforth restrict attention to the nontrivial case $R_{\mathbf{d}^{*}}(\mathbf{f}(\boldsymbol{D}), C)>0$.

Suppose, to the contrary of Lemma 40 that $\tilde{\mathrm{R}}_{\mathbf{f}, \text { max-exc }}^{\dagger}(\boldsymbol{D}, C)$ is strictly smaller than $\mathrm{R}_{\mathbf{d}^{*}}(\mathbf{f}(\boldsymbol{D}), C)$ and, therefore, there exists some $\gamma>0$ such that

$$
\tilde{\mathbf{R}}_{\mathbf{f}, \text { max-exc }}^{\dagger}(\boldsymbol{D}, C) \leq \mathbf{R}_{\mathbf{d}^{*}}(\mathbf{f}(\boldsymbol{D}), C)-\gamma .
$$

By the continuity and monotonicity of $\mathbf{R}_{\mathbf{d}^{*}}(\mathbf{f}(\boldsymbol{D}), C)$ and each $\mathrm{f}_{\ell}$, there exists some distortion tuple $\boldsymbol{D}^{\prime}$ such that

$$
\mathbf{R}_{\mathbf{d}^{*}}\left(\mathbf{f}\left(\boldsymbol{D}^{\prime}\right), C\right)=\mathrm{R}_{\mathbf{d}^{*}}(\mathbf{f}(\boldsymbol{D}), C)-\frac{\gamma}{2}
$$

where $D_{\ell}^{\prime}>D_{\ell}$ for all $\ell \in \mathcal{L}$.

Now recall Definition 9 and the operational meaning of $\tilde{\mathrm{R}}_{\mathrm{f} \text {,max-exc }}^{\dagger}(\boldsymbol{D}, C)$. There exists a sequence of $\left(n, \mathcal{M}_{\mathrm{c}}^{(n)}, \mathcal{M}^{(n)}\right)$-codes satisfying (5a), (5b) and [25). On combining (5b), 50) and (91), we see that the delivery-phase rates of this sequence of codes satisfy

$$
\limsup _{n \rightarrow \infty} \frac{1}{n} \log \left|\mathcal{M}^{(n)}\right| \leq \mathbf{R}_{\mathbf{d}^{*}}\left(\mathbf{f}\left(\boldsymbol{D}^{\prime}\right), C\right)-\frac{\gamma}{2}
$$

Now consider the excess-distortion performance of the sequence of $\left(n, \mathcal{M}_{\mathrm{c}}^{(n)}, \mathcal{M}^{(n)}\right)$-codes w.r.t. the separable distortion functions $\mathbf{d}^{*}$. Let

$$
\mathcal{B}_{n}=\bigcup_{\ell \in \mathcal{L}}\left\{\overline{\mathrm{d}}_{\ell}^{*}\left(\hat{X}_{\ell}^{n}, X_{\ell}^{n}\right) \geq \mathrm{f}_{\ell}\left(D_{\ell}^{\prime}\right)\right\}
$$

and let $\mathcal{B}_{n}^{c}$ denote the complement of $\mathcal{B}_{n}$. Notice that we have

$$
\mathcal{B}_{n}=\bigcup_{\ell \in \mathcal{L}}\left\{\overline{\mathrm{fd}}_{\ell}\left(\hat{X}_{\ell}^{n}, X_{\ell}^{n}\right) \geq D_{\ell}^{\prime}\right\} .
$$

Since the asymptotic delivery-phase rate is strictly smaller than the informational RDC function (92), the strong converse in Theorem 17 yields

$$
\limsup _{n \rightarrow \infty} \mathbb{P}\left[\mathcal{B}_{n}\right]=1
$$

Let

$$
\zeta=\min _{\ell \in \mathcal{L}}\left(D_{\ell}^{\prime}-D_{\ell}\right)
$$

We now have

$$
\begin{aligned}
& \mathbb{E}\left[\max _{\ell \in \mathcal{L}}\left(\overline{\mathrm{fd}}_{\ell}\left(\hat{X}_{\ell}^{n}, X_{\ell}^{n}\right)-D_{\ell}\right)\right] \\
& =\mathbb{E}\left[\max _{\ell \in \mathcal{L}}\left(\overline{\mathrm{fd}}_{\ell}\left(\hat{X}_{\ell}^{n}, X_{\ell}^{n}\right)-D_{\ell}\right) \mid \mathcal{B}_{n}\right] \mathbb{P}\left[\mathcal{B}_{n}\right] \\
& \quad+\mathbb{E}\left[\max _{\ell \in \mathcal{L}}\left(\overline{\mathrm{fd}}_{\ell}\left(\hat{X}_{\ell}^{n}, X_{\ell}^{n}\right)-D_{\ell}\right) \mid \mathcal{B}_{n}^{c}\right] \mathbb{P}\left[\mathcal{B}_{n}^{c}\right] \\
& \geq \mathbb{E}\left[\max _{\ell \in \mathcal{L}}\left(\overline{\mathrm{fd}}_{\ell}\left(\hat{X}_{\ell}^{n}, X_{\ell}^{n}\right)-D_{\ell}\right) \mid \mathcal{B}_{n}\right] \mathbb{P}\left[\mathcal{B}_{n}\right] \\
& -\left(\min _{\ell \in \ell} D_{\ell}\right) \mathbb{P}\left[\mathcal{B}_{n}^{c}\right] \\
& \geq \\
& \geq \zeta \mathbb{P}\left[\mathcal{B}_{n}\right]-\left(\min _{\ell \in \ell} D_{\ell}\right) \mathbb{P}\left[\mathcal{B}_{n}^{c}\right],
\end{aligned}
$$

where (a) follows because $\overline{\mathrm{fd}}_{\ell}\left(\hat{X}_{\ell}^{n}, X_{\ell}^{n}\right)$ is nonnegative; and (b) follows because, conditioned on $\mathcal{B}_{n}$, there must exist at least one $\ell^{\prime} \in \mathcal{L}$ such that

$$
\overline{\mathrm{fd}}_{\ell^{\prime}}\left(\hat{X}_{\ell^{\prime}}^{n}, X_{\ell^{\prime}}^{n}\right) \geq D_{\ell^{\prime}}^{\prime}>D_{\ell^{\prime}}
$$

and thus

$$
\max _{\ell \in \mathcal{L}}\left(\overline{\mathrm{fd}}_{\ell}\left(\hat{X}_{\ell}^{n}, X_{\ell}^{n}\right)-D_{\ell}\right) \geq \overline{\mathrm{fd}}_{\ell^{\prime}}\left(\hat{X}_{\ell^{\prime}}^{n}, X_{\ell^{\prime}}^{n}\right)-D_{\ell^{\prime}}>\zeta .
$$


Finally, we have

$$
\begin{aligned}
0 & \stackrel{\mathrm{a}}{=} \limsup _{n \rightarrow \infty} \mathbb{E}\left[\max _{\ell \in \mathcal{L}}\left(\overline{\mathrm{fd}}_{\ell}\left(\hat{X}_{\ell}^{n}, X_{\ell}^{n}\right)-D_{\ell}\right)\right] \\
& \geq \limsup _{n \rightarrow \infty}\left[\zeta \mathbb{P}\left[\mathcal{B}_{n}\right]-\left(\min _{\ell \in \mathcal{L}} D_{\ell}\right) \mathbb{P}\left[\mathcal{B}_{n}^{c}\right]\right] \\
& >0,
\end{aligned}
$$

where (a) follows from (25), (b) follows from [93), and (c) follows because $\mathbb{P}\left[\mathcal{B}_{n}\right] \rightarrow 1$ by the strong converse Theorem 17 and $\zeta>0$. The above contradiction implies that $\tilde{R}_{\mathbf{f}, \text { max-exc }}^{\dagger}(\boldsymbol{D}, C)$ cannot be strictly smaller than $\mathrm{R}_{\mathbf{d}^{*}}(\mathbf{f}(\boldsymbol{D}), C)$.

To complete the proof of Theorem $\mathbb{I}$ we need only combine the above lemmas:

$$
\begin{aligned}
\tilde{\mathrm{R}}_{\mathbf{f}, \text { max-exc }}^{\dagger}(D, C) & \stackrel{\mathrm{a}}{\leq} \mathrm{R}_{\mathbf{f}}^{\ddagger}(\boldsymbol{D}, C) \\
& \stackrel{\mathrm{b}}{=} \mathrm{R}_{\mathbf{d}^{*}}^{\ddagger}(\mathbf{f}(\boldsymbol{D}), C) \\
& \stackrel{\mathrm{c}}{=} \mathrm{R}_{\mathbf{d}^{*}}(\mathbf{f}(\boldsymbol{D}), C) \\
& \stackrel{\mathrm{d}}{\leq} \tilde{\mathrm{R}}_{\mathbf{f}, \text { max-exc }}^{\dagger}(\boldsymbol{D}, C),
\end{aligned}
$$

where (a) uses Lemma 39, (b) uses Lemma 38, (c) uses Lemma 37, and (d) uses Lemma 40.

APPENDIX J

PROOF OF THEOREM 21

Fix $\boldsymbol{D}=(D, D, \ldots, D)$ for some $D \geq 0$, and consider any tuple $(U, \hat{\boldsymbol{X}})$ satsifying 21). Fix $\mathcal{S} \subseteq \mathcal{L}$ and let $S:=|\mathcal{S}|$. Then

$$
\begin{array}{rl}
\max _{\ell \in \mathcal{L}} & I\left(X_{\ell} ; \hat{X}_{\ell} \mid U\right) \\
& \geq \max _{\ell \in \mathcal{S}}\left[h\left(X_{\ell} \mid U\right)-h\left(X_{\ell} \mid \hat{X}_{\ell}\right)\right] \\
& \geq \frac{1}{S} h\left(X_{\mathcal{S}} \mid U\right)-\frac{1}{2} \log (2 \pi e D) \\
& \geq \frac{1}{S}\left(\frac{1}{2} \log \left((2 \pi e)^{S} \operatorname{det} \boldsymbol{K}_{X_{\mathcal{S}}}\right)-C\right)-\frac{1}{2} \log (2 \pi e D) \\
& =\frac{1}{2 S} \log \frac{\operatorname{det} \boldsymbol{K}_{X_{\mathcal{S}}}}{D^{S}}-\frac{C}{S} .
\end{array}
$$

Step (a) follows because

$$
\begin{aligned}
h\left(X_{\ell} \mid \hat{X}_{\ell}\right) & \stackrel{\text { a. }}{=} h\left(X_{\ell}-\hat{X}_{\ell} \mid \hat{X}_{\ell}\right) \\
& \stackrel{\text { a.2 }}{\leq} h\left(\mathcal{N}\left(0, \mathbb{E}\left(\hat{X}_{\ell}-X_{\ell}\right)^{2}\right)\right) \\
& \stackrel{\text { a.3 }}{\leq} h(\mathcal{N}(0, D)) \\
& \stackrel{\text { a.4 }}{\leq} \frac{1}{2} \log (2 \pi e D),
\end{aligned}
$$

where (a.1) follows by the translation property of differential entropy [30, Thm. 10.18]; (a.2) uses the fact that the normal distribution maximises differential entropy for a given second moment [30. Thm. 10.43], and (a.3) invokes the distortion constraint in 21. Moreover, for the first term, we have

$$
\max _{\ell \in \mathcal{S}} h\left(X_{\ell} \mid U\right) \stackrel{\text { a.5 }}{\geq} \frac{1}{S} \sum_{\ell \in \mathcal{S}} h\left(X_{\ell} \mid U\right) \stackrel{\text { a.6 }}{\geq} \frac{1}{S} h\left(X_{\mathcal{S}} \mid U\right)
$$

where (a.5) follows because the maximum cannot be smaller than the average, and (a.6) follows by the independence bound for differential entropy [30, Thm. 10.34]

Step (b) follows from the cache capacity constraint in 21

$$
\begin{aligned}
C & \geq I(\boldsymbol{X} ; U) \geq I\left(X_{\mathcal{S}} ; U\right) \\
& =h\left(X_{\mathcal{S}}\right)-h\left(X_{\mathcal{S}} \mid U\right) \\
& =\frac{1}{2} \log \left((2 \pi e)^{S} \operatorname{det} \boldsymbol{K}_{X_{\mathcal{S}}}\right)-h\left(X_{\mathcal{S}} \mid U\right)
\end{aligned}
$$


APPENDIX K

ProOF OF THEOREM 22

A. Case 1: $(D, C) \in \mathcal{S}_{1}$

If $(D, C) \in \mathcal{S}_{1}$, then it trivially follows from the definition of $\mathrm{R}_{\mathrm{G}, X_{1} X_{2}}(D, D)$ that $\mathrm{R}_{\mathrm{G}}(D, D, C)=0$.

B. Case 2: $(D, C) \in \mathcal{S}_{2}$

Since $\mathrm{R}_{\mathrm{G}, X_{1} X_{2}}(D, D)$ is strictly decreasing in $D$, it follows that for a given $C \leq \mathrm{R}_{\mathrm{G}, X_{1} X_{2}}(D, D)$ the distortion $D$ must satisfy

$$
0<D \leq 2^{-C} \sqrt{1-\rho^{2}} .
$$

Define

$$
\alpha=1-\rho-2^{-C} \sqrt{1-\rho^{2}}
$$

and note that $0 \leq \alpha<1-\rho$ for all finite

$$
C>\frac{1}{2} \log \frac{1+\rho}{1-\rho} .
$$

Now let $W, N_{1}, N_{2}, \tilde{N}_{1}, \tilde{N}_{2}, Z_{1}$ and $Z_{2}$ be mutually independent standard Gaussians $\mathcal{N}(0,1)$, and notice that our bivariate Gaussian source $\left(X_{1}, X_{2}\right)$ can be written as

$$
X_{i}=\sqrt{\rho} W+\sqrt{\alpha} N_{i}+\sqrt{1-\rho-D-\alpha} \tilde{N}_{i}+\sqrt{D} Z_{i}, \quad i=1,2
$$

Choose $U=\left(U_{1}, U_{2}\right)$, where

$$
U_{i}=\sqrt{\rho} W+\sqrt{\alpha} N_{i}, \quad i=1,2 .
$$

Define the reconstructions $\hat{X}_{1}$ and $\hat{X}_{2}$ to be

$$
\hat{X}_{i}:=U_{i}+\sqrt{1-\rho-\alpha-D} \tilde{N}_{i}, \quad i=1,2 .
$$

We notice that

$$
X_{1} \leftrightarrow \hat{X}_{1} \leftrightarrow U_{1} \leftrightarrow U \leftrightarrow U_{2} \leftrightarrow \hat{X}_{2} \leftrightarrow X_{2}
$$

forms a Markov chain. Additionally,

$$
\begin{aligned}
& I\left(X_{1}, X_{2} ; U\right) \\
& =h\left(X_{1}, X_{2}\right)-h\left(X_{1}, X_{2} \mid U\right) \\
& \stackrel{\mathrm{a}}{=} h\left(X_{1}, X_{2}\right)-h\left(X_{1} \mid U\right)-h\left(X_{2} \mid U\right) \\
& \stackrel{\mathrm{b}}{=} h\left(X_{1}, X_{2}\right)-h\left(X_{1} \mid U_{1}\right)-h\left(X_{2} \mid U_{2}\right) \\
& =h\left(X_{1}, X_{2}\right)-2 h\left(X_{1} \mid U_{1}\right) \\
& \stackrel{\mathrm{c}}{=} h\left(X_{1}, X_{2}\right)-2 h\left(X_{1}-U_{1} \mid U_{1}\right) \\
& =\frac{1}{2} \log \left((2 \pi \mathrm{e})^{2}\left(1-\rho^{2}\right)\right)-\log (2 \pi \mathrm{e}(1-\rho-\alpha)) \\
& =\frac{1}{2} \log \frac{1-\rho^{2}}{(1-\rho-\alpha)^{2}} \\
& \stackrel{\mathrm{d}}{=} C
\end{aligned}
$$

where (a) and (b) follow from 95, (c) follows by symmetry, and (d) substitutes 947. Similarly,

$$
\begin{aligned}
I\left(X_{1} ; \hat{X}_{1} \mid U\right) & =h\left(X_{1} \mid U\right)-h\left(X_{1} \mid \hat{X}_{1}, U\right) \\
& \stackrel{\mathrm{a}}{=} h\left(X_{1} \mid U_{1}\right)-h\left(X_{1} \mid \hat{X}_{1}\right) \\
& =h\left(X_{1}-U_{1} \mid U_{1}\right)-h\left(X_{1}-\hat{X}_{1} \mid \hat{X}_{1}\right) \\
& =\frac{1}{2} \log (2 \pi \mathrm{e}(1-\rho-\alpha))-\frac{1}{2} \log (2 \pi \mathrm{e} D) \\
& =\frac{1}{2} \log \left(\frac{1-\rho-\alpha}{D}\right) \\
& =\frac{1}{4} \log \left(\frac{1-\rho^{2}}{D^{2}}\right)-\frac{C}{2},
\end{aligned}
$$

where (a) uses the Markov chain (95) and (b) substitutes 947. Finally, we notice that the above achievable rate is equal to the superuser lower bound from Theorem 21. 
C. Case 3: $(D, C) \in \mathcal{S}_{3}$

Let

$$
\alpha=\frac{1}{2}(1+\rho)\left(1-2^{-2 C}\right),
$$

and note that $0 \leq \alpha \leq \rho$. Now let $W, \tilde{W}, Z_{1}, Z_{2}, N_{1}$ and $N_{2}$ be mutually independent standard Gaussians $\mathcal{N}(0,1)$. Choose

$$
U=\sqrt{\alpha} W+\sqrt{\rho-\alpha} \tilde{W}
$$

and

$$
\hat{X}_{i}=\sqrt{\rho} W+\sqrt{1-\rho-D} Z_{i}, \quad i=1,2 .
$$

We may now write our bivariate Gaussian source $\left(X_{1}, X_{2}\right)$ as

$$
X_{i}=\hat{X}_{i}+\sqrt{D} N_{i}, \quad i=1,2 .
$$

The pair $\left(X_{1}, U\right)$ and the pair $\left(X_{2}, U\right)$ are both zero mean bivariate Gaussians with identical covariance matrices

$$
\boldsymbol{K}_{X_{1}, U}=\boldsymbol{K}_{X_{2}, U}=\left[\begin{array}{cc}
1 & \sqrt{\alpha \rho} \\
\sqrt{\alpha \rho} & \rho
\end{array}\right] .
$$

Similarly, $\left(X_{1}, X_{2}, U\right)$ is a zero mean multivariate normal with the covariance matrix

$$
\boldsymbol{K}_{X_{1} X_{2} U}=\left[\begin{array}{ccc}
1 & \rho & \sqrt{\alpha \rho} \\
\rho & 1 & \sqrt{\alpha \rho} \\
\sqrt{\alpha \rho} & \sqrt{\alpha \rho} & \rho
\end{array}\right] .
$$

Thus,

$$
\begin{aligned}
I\left(X_{1}, X_{2} ; U\right) & =h\left(X_{1}, X_{2}\right)+h(U)-h\left(X_{1}, X_{2}, U\right) \\
& =\frac{1}{2} \log \left((2 \pi e)^{2} \operatorname{det} \boldsymbol{K}_{X_{1} X_{2}}\right)+\frac{1}{2} \log (2 \pi e \rho) \\
& \quad-\frac{1}{2} \log \left((2 \pi e)^{3} \operatorname{det} \boldsymbol{K}_{X_{1} X_{2} U}\right) \\
& =\frac{1}{2} \log \frac{1+\rho}{1+\rho-2 \alpha} \\
& =C
\end{aligned}
$$

and

$$
\begin{aligned}
I\left(X_{1} ; \hat{X}_{1} \mid U\right) & =h\left(X_{1} \mid U\right)-h\left(X_{1} \mid \hat{X}_{1}, U\right) \\
& =h\left(X_{1}, U\right)-h(U)-h\left(X_{1} \mid \hat{X}_{1}\right) \\
& =\frac{1}{2} \log \left((2 \pi e)^{2} \operatorname{det} \boldsymbol{K}_{X_{1} U}\right)-\frac{1}{2} \log (2 \pi e \rho) \\
& =\frac{1}{2} \log (2 \pi e D) \\
& =\frac{1}{2} \log \frac{1-\alpha}{D} .
\end{aligned}
$$

D. Case 4: $(D, C) \in \mathcal{S}_{4}$

Suppose that $(D, C) \in \mathcal{S}_{4}$. Since $(D, C)$ lies below the Gaussian joint $\mathrm{RD}$ function $\mathrm{R}_{\mathrm{G}, X_{1} X_{2}}(D, D)$, it follows that for any given distortion $D \in[1-\rho, 1]$ the cache capacity $C$ must lie within

$$
0 \leq C \leq \frac{1}{2} \log \frac{1+\rho}{2 D-1+\rho} .
$$

Define

$$
\alpha=\frac{1}{2}(1+\rho)\left(1-2^{-2 C}\right)
$$

and

$$
\beta=1-\alpha-D,
$$

where we notice that

$$
0 \leq \alpha, \beta \leq 1-D \quad \text { and } \quad \alpha+\beta=1-D \leq \rho .
$$


In this case, we may write

$$
X_{i}=\sqrt{\alpha} A+\sqrt{\beta} B+\sqrt{\rho-(\alpha+\beta)} W+\sqrt{1-\rho} N_{i}, \quad i=1,2,
$$

where $A, B, W, N_{1}$ and $N_{2}$ are mutually independent standard Gaussians $\mathcal{N}(0,1)$. Now let

$$
U=\sqrt{\alpha} A
$$

and

$$
\hat{X}_{1}=\hat{X}_{2}=\hat{X}:=U+\sqrt{\beta} B .
$$

Here $\left(X_{1}, X_{2}, U\right)$ is a zero mean multivariate Gaussian with covariance matrix

$$
\boldsymbol{K}_{X_{1}, X_{2}, U}=\left[\begin{array}{ccc}
1 & \rho & \alpha \\
\rho & 1 & \alpha \\
\alpha & \alpha & \alpha .
\end{array}\right]
$$

Then,

$$
\begin{aligned}
I\left(X_{1}, X_{2} ; U\right)= & h\left(X_{1}, X_{2}\right)-h\left(X_{1}, X_{2}, U\right)-h(U) \\
= & \frac{1}{2} \log \left((2 \pi \mathrm{e})^{2} \operatorname{det} \boldsymbol{K}_{X_{1}, X_{2}}\right) \\
& \quad-\frac{1}{2} \log \left((2 \pi \mathrm{e})^{3} \operatorname{det} \boldsymbol{K}_{X_{1}, X_{2}, U}\right) \\
& +\frac{1}{2} \log (2 \pi \\
= & \frac{1}{2} \log \frac{1+\rho}{1+\rho-2 \alpha} \\
= & C .
\end{aligned}
$$

Moreover,

$$
\begin{aligned}
I\left(X_{1} ; \hat{X} \mid U\right) & =h\left(X_{1} \mid U\right)-h\left(X_{1} \mid U, \hat{X}\right) \\
& =h\left(X_{1} \mid U\right)-h\left(X_{1} \mid \hat{X}\right) \\
& =\frac{1}{2} \log (2 \pi \mathrm{e}(1-\alpha))-\frac{1}{2} \log (2 \pi \mathrm{e}(1-\alpha-\beta)) \\
& =\frac{1}{2} \log \frac{1-\alpha}{D} .
\end{aligned}
$$

\section{APPENDIX L}

Proof of Theorem 26 (Outline)

Choose $(U, \hat{\boldsymbol{X}}, \tilde{\boldsymbol{X}})$ such that $28 \mathrm{a}$ and $28 \mathrm{~b}$ hold. We need only find a scheme that has an arbitrarily small average error probability whenever

$$
\begin{aligned}
& C \geq I(U ; \boldsymbol{X})-I\left(U ; \tilde{X}_{\ell_{2}}\right) \\
& R \geq I\left(U, \boldsymbol{X} ; \tilde{X}_{\ell_{2}}\right)+I\left(\boldsymbol{X} ; \hat{X}_{\ell_{1}} \mid U, \tilde{X}_{\ell_{2}}\right)
\end{aligned}
$$

holds for all $\left(\ell_{1}, \ell_{2}\right) \in \mathcal{L}_{1} \times \mathcal{L}_{2}$.

\section{A. Code Construction}

Fix $\epsilon>0$ arbitrarily small. Generate a $U$-codebook

$$
\left\{U^{n}\left(m_{u}\right)=\left(U_{1}\left(m_{u}\right), U_{2}\left(m_{u}\right), \ldots, U_{n}\left(m_{u}\right)\right)\right\}
$$

indexed by

$$
m_{u}=1,2, \ldots, 2^{n(I(\boldsymbol{X} ; U)+\epsilon)}
$$

by randomly selecting symbols from $\mathcal{U}$ in an iid manner using $U \sim p_{U}$. For each index $\ell_{2}$, generate an $\tilde{X}_{\ell_{2}}$-codebook

$$
\left\{\tilde{X}_{\ell_{2}}^{n}\left(m_{\ell_{2}}\right)=\left(\tilde{X}_{\ell_{2}, 1}\left(m_{\ell_{2}}\right), \ldots, \tilde{X}_{\ell_{2}, n}\left(m_{\ell_{2}}\right)\right)\right\}
$$

indexed by

$$
m_{\ell_{2}}=1,2, \ldots, 2^{n\left(I\left(U, \boldsymbol{X} ; \tilde{X}_{\ell_{2}}\right)+\epsilon\right)}
$$


by selecting symbols from $\tilde{\mathcal{X}}_{\ell_{2}}$ iid $\tilde{X}_{\ell_{2}} \sim p_{\tilde{X}_{\ell_{2}}}$. Finally, for each $\left(\ell_{2}, m_{u}, m_{\ell_{2}}\right)$, generate an $\hat{X}_{\ell_{1}}$-codebook

$$
\left\{\hat{X}_{\ell_{1}}^{n}\left(m_{u}, m_{\ell_{2}}, \ell_{2}, m_{\ell_{1}}\right)=\left(\hat{X}_{\ell_{1}, 1}\left(m_{u}, m_{\ell_{2}}, \ell_{2}, m_{\ell_{1}}\right), \ldots, \hat{X}_{\ell_{1}, n}\left(m_{u}, m_{\ell_{2}}, \ell_{2}, m_{\ell_{1}}\right)\right)\right\}
$$

indexed by

$$
m_{\ell_{1}}=1,2, \ldots, 2^{n\left(I\left(\boldsymbol{X} ; \hat{X}_{\ell_{1}} \mid U, \tilde{X}_{2}\right)+\epsilon\right)}
$$

by selecting symbols from $\hat{\mathcal{X}}_{\ell_{1}}$ independently (in a memoryless manner) according to

$$
\prod_{i=1}^{n} p_{\hat{X}_{\ell_{1}} \mid U, \tilde{X}_{\ell_{2}}}\left(\cdot \mid u_{i}\left(m_{u}\right), \tilde{x}_{\ell_{2} i}\left(m_{\ell_{2}}\right)\right) .
$$

\section{B. Cache Encoder at Server}

Find a tuple of indices $m_{u}$ and $\left(m_{\ell_{2}} ; \ell_{2} \in \mathcal{L}_{2}\right)$ such that

$$
\left(U^{n}\left(m_{u}\right), \boldsymbol{X}^{n}, \tilde{X}_{\ell_{2}}^{n}\left(m_{\ell_{2}}\right)\right)
$$

is jointly typical for every $\ell_{2} \in \mathcal{L}_{2}$. If there are one or more index tuples pick one uniformly at random; otherwise, declare an error. Represent $m_{u}$ as a string of binary bits, and let $m_{c}$ denote the first $n C$ bits of $m_{u}$.

\section{Delivery-Phase Encoder at Server}

Given $\left(m_{u}, m_{\ell_{2}}\right)$, find $m_{\ell_{1}}$ such that

$$
\left(U^{n}\left(m_{u}\right), \boldsymbol{X}^{n}, \tilde{X}_{\ell_{2}}^{n}\left(m_{\ell_{2}}\right), \hat{X}_{\ell_{1}}^{n}\left(m_{u}, m_{\ell_{2}}, \ell_{2}, m_{\ell_{1}}\right)\right)
$$

is jointly typical. If there are one or more indices, pick one uniformly at random and set $m=\left(m_{\ell_{1}}, m_{\ell_{2}}\right)$. If there is no such index tuple declare an error.

\section{Delivery-Phase Decoders}

1) User 1: Find an index $\hat{m}_{u}$ with the same first $n C$ bits as in $m_{\mathrm{c}}$ such that

$$
\left(U^{n}\left(\hat{m}_{u}\right), \tilde{X}_{\ell_{2}}^{n}\left(m_{\ell_{2}}\right)\right)
$$

is jointly typical. If there is no such index declare error. If there are multiple such indices choose one uniformly at random. Output $\hat{X}_{\ell_{1}}^{n}\left(\hat{m}_{u}, m_{\ell_{2}},{\underset{\tilde{\ell}}{2}}_{2}, m_{\ell_{1}}\right)$.

2) User 2: Output $\tilde{X}_{\ell_{2}}^{n}\left(m_{\ell_{2}}\right)$.

\section{APPENDIX M}

PROOF OF COROLLARY 30

Starting from 33 , one can write

$$
\begin{aligned}
& \mathrm{R}^{\dagger}(\boldsymbol{D}, \mathbf{0}, C) \\
& \geq \underline{\mathrm{R}}(\boldsymbol{D}, \mathbf{0}, C) \\
& \geq 1+\max _{\left(\ell_{1}, \ell_{2}\right)} \max \left\{0, R_{X_{\ell_{1}} \mid X_{\ell_{2}}}\left(D_{\ell_{1}}\right)-C\right\} \\
& \geq 1+\max \left\{0, R_{X_{\ell} \mid X_{\ell^{\prime}}}(D)-C, R_{X_{\ell^{\prime}} \mid X_{\ell}}(D)-C\right\} \\
& \geq 1+\max \{0, h(\rho)-h(D)-C\}
\end{aligned}
$$

where 97) is because min - max is larger than or equal to max - min and (99) is because the conditional rate-distortion function of a doubly symmetric source is given by $h(\rho)-h(D)$.

Also, we specialise (35) by choosing $U=\left(\hat{X}_{\ell}, \hat{X}_{\ell^{\prime}}\right)$ to obtain

$$
\begin{aligned}
& R^{\dagger}(\boldsymbol{D}, \mathbf{0}, C) \\
& \leq \overline{\mathrm{R}}(\boldsymbol{D}, \mathbf{0}, C) \\
& \leq \min _{\hat{\boldsymbol{X}}} \max _{\ell_{2}} \max \left\{I\left(\boldsymbol{X} ; \hat{X}_{\ell}, \hat{X}_{\ell^{\prime}}, X_{\ell_{2}}\right)-C, H\left(X_{\ell_{2}}\right)\right\} \\
& =1+\min _{\hat{\boldsymbol{X}}} \max \left\{0, I\left(\boldsymbol{X} ; \hat{X}_{\ell}, \hat{X}_{\ell^{\prime}} \mid X_{\ell^{\prime}}\right)-C,\right.
\end{aligned}
$$




$$
\leq\left\{\begin{array}{c}
\left.I\left(\boldsymbol{X} ; \hat{X}_{\ell}, \hat{X}_{\ell^{\prime}} \mid X_{\ell}\right)-C\right\} \\
\text { If } D \leq D^{\star} \\
1+\left(h(D)-\rho-(1-\rho) h\left(\frac{2 D-\rho}{2(1-\rho)}\right)-C\right)^{+} \\
\text {If } D^{\star}<D \leq \frac{1}{2}
\end{array}\right.
$$

where $D^{\star}$ is defined in (39) and [103) follows by the choice of $\hat{\boldsymbol{X}}$ that is made in the proof of [19. Theorem 3].

\section{APPENDIX N}

\section{PROOF OF THEOREM 31}

For any $\epsilon>0$, let the distortion vector $\epsilon=(\epsilon, \ldots, \epsilon)$. We have

$$
\begin{aligned}
& (R(\boldsymbol{D}, \boldsymbol{\epsilon}, C)+C) \\
\geq & H\left(M_{\ell_{1}, \ell_{2}}^{(n)}, M_{c}^{(n)}\right) \\
\geq & I\left(M_{\ell_{1}, \ell_{2}}^{(n)}, M_{c}^{(n)} ; \boldsymbol{X}^{n}\right) \\
& =\sum_{i=1}^{n} I\left(M_{\ell_{1}, \ell_{2}}^{(n)}, M_{c}^{(n)}, \boldsymbol{X}^{i-1} ; \boldsymbol{X}_{i}\right) \\
& =\sum_{i=1}^{n} I\left(\hat{X}_{\ell_{1}}^{n}, \tilde{X}_{\ell_{2}}^{n}, M_{c}^{(n)}, \boldsymbol{X}^{i-1} ; \boldsymbol{X}_{i}\right) \\
\geq & \sum_{i=1}^{n} I\left(\hat{X}_{\ell_{1}}^{n}, X_{\ell_{2}}^{n}, M_{c}^{(n)}, \boldsymbol{X}^{i-1} ; \boldsymbol{X}_{i}\right)-n \delta_{1}(\epsilon) \\
= & \sum_{i=1}^{n} I\left(\hat{X}_{\ell_{1}}^{n}, X_{\ell_{2}, i}, X_{\ell_{2}, i+1}^{n}, M_{c}^{(n)}, \boldsymbol{X}^{i-1} ; \boldsymbol{X}_{i}\right)-n \delta_{1}(\epsilon) \\
\geq & \sum_{i=1}^{n} I\left(\hat{X}_{\ell_{1}, i}, X_{\ell_{2}, i}, U_{\ell_{2}, i} ; \boldsymbol{X}_{i}\right)-n \delta_{1}(\epsilon) \\
= & n I\left(\hat{X}_{\ell_{1}, Q}, X_{\ell_{2}, Q}, U_{\ell_{2}, Q} ; \boldsymbol{X}_{Q} \mid Q\right)-n \delta_{1}(\epsilon) \\
= & n I\left(\hat{X}_{\ell_{1}, Q}, X_{\ell_{2}, Q}, U_{\ell_{2}, Q}, Q ; \boldsymbol{X}_{Q}\right)-n \delta_{1}(\epsilon)
\end{aligned}
$$

where we have defined $U_{\ell_{2}, i}=\left(M_{c}^{(n)}, X_{\ell_{2}, i+1}^{n}, \boldsymbol{X}^{i-1}\right)$, and $Q$ is a random variable that is independent of everything else and takes values in $\{1, \ldots, n\}$ uniformly at random. In the above chain of inequalities, (107) is because $\tilde{X}_{\ell_{2}}^{n}$ and $\hat{X}_{\ell_{1}}^{n}$ are functions of $M_{\ell_{1}, \ell_{2}}^{(n)}$ and $\left(M_{c}^{(n)}, M_{\ell_{1}, \ell_{2}}^{(n)}\right)$, respectively, and $(108)$ is because $\bar{d}_{\ell_{2}}\left(X_{\ell_{2}}^{n}, \tilde{X}_{\ell_{2}}^{n}\right) \leq \epsilon$. Here, $\delta_{1}(\epsilon) \rightarrow 0$ as $\epsilon \rightarrow 0$.

Similarly, we have

$$
\begin{aligned}
& R(\boldsymbol{D}, \boldsymbol{\epsilon}, C) \\
\geq & I\left(M_{\ell_{1}, \ell_{2}}^{(n)} ; M_{c}^{(n)}, \boldsymbol{X}^{n}\right) \\
= & I\left(M_{\ell_{1}, \ell_{2}}^{(n)}, \tilde{X}_{\ell_{2}}^{n} ; M_{c}^{(n)}, \boldsymbol{X}^{n}\right) \\
\geq & I\left(M_{\ell_{1}, \ell_{2}}^{(n)}, X_{\ell_{2}}^{n} ; M_{c}^{(n)}, \boldsymbol{X}^{n}\right)-n \delta_{2}(\epsilon) \\
\geq & I\left(X_{\ell_{2}}^{n} ; M_{c}^{(n)}, \boldsymbol{X}^{n}\right)+I\left(M_{\ell_{1}, \ell_{2}}^{(n)} ; M_{c}^{(n)}, \boldsymbol{X}^{n} \mid X_{\ell_{2}}^{n}\right)-n \delta_{2}(\epsilon) \\
\geq & I\left(X_{\ell_{2}}^{n} ; M_{c}^{(n)}, \boldsymbol{X}^{n}\right)+I\left(M_{\ell_{1}, \ell_{2}}^{(n)} ; \boldsymbol{X}^{n} \mid M_{c}^{(n)}, X_{\ell_{2}}^{n}\right)-n \delta_{2}(\epsilon) \\
\geq & I\left(X_{\ell_{2}}^{n} ; M_{c}^{(n)}, \boldsymbol{X}^{n}\right)+I\left(\hat{X}_{\ell_{1}}^{n} ; \boldsymbol{X}^{n} \mid M_{c}^{(n)}, X_{\ell_{2}}^{n}\right)-n \delta_{2}(\epsilon) \\
= & \sum_{i=1}^{n} I\left(X_{\ell_{2}, i} ; M_{c}^{(n)}, \boldsymbol{X}^{n} \mid X_{\ell_{2}, i+1}^{n}\right) \\
& +\sum_{i=1}^{n} I\left(\hat{X}_{\ell_{1}}^{n} ; \boldsymbol{X}_{i} \mid M_{c}^{(n)}, X_{\ell_{2}}^{n}, \boldsymbol{X}^{i-1}\right)-n \epsilon \\
= & \sum_{i=1}^{n} I\left(X_{\ell_{2}, i} ; M_{c}^{(n)}, \boldsymbol{X}^{n}, X_{\ell_{2}, i+1}^{n}\right)
\end{aligned}
$$




$$
\begin{aligned}
& +\sum_{i=1}^{n} I\left(\hat{X}_{\ell_{1}}^{n} ; \boldsymbol{X}_{i} \mid M_{c}^{(n)}, X_{\ell_{2}}^{n}, \boldsymbol{X}^{i-1}\right)-n \epsilon \\
\geq & \sum_{i=1}^{n} I\left(X_{\ell_{2}, i} ; M_{c}^{(n)} \boldsymbol{X}^{i-1} X_{\ell_{2}, i+1}^{n}\right) \\
& +\sum_{i=1}^{n} I\left(\hat{X}_{\ell_{1}, i} ; \boldsymbol{X}_{i} \mid M_{c}^{(n)}, \boldsymbol{X}^{i-1}, X_{\ell_{2}, i+1}^{n}, X_{\ell_{2}, i}\right)-n \delta_{2}(\epsilon) \\
= & \sum_{i=1}^{n} I\left(X_{\ell_{2}, i} ; U_{\ell_{2}, i}\right) \\
& +\sum_{i=1}^{n} I\left(\hat{X}_{\ell_{1}, i} ; \boldsymbol{X}_{i} \mid U_{\ell_{2}, i}, X_{\ell_{2}, i}\right)-n \delta_{2}(\epsilon) \\
= & n I\left(X_{\ell_{2}, Q} ; U_{\ell_{2}, Q} \mid Q\right) \\
& +n I\left(\hat{X}_{\ell_{1}, Q} ; \boldsymbol{X}_{Q} \mid U_{\ell_{2}, Q}, X_{\ell_{2}, Q}, Q\right)-n \delta_{2}(\epsilon) \\
= & n I\left(X_{\ell_{2}, Q} ; U_{\ell_{2}, Q} Q\right) \\
& +n I\left(\hat{X}_{\ell_{1}, Q} ; \boldsymbol{X}_{Q} \mid U_{\ell_{2}, Q}, Q, X_{\ell_{2}, Q}\right)-n \delta_{2}(\epsilon)
\end{aligned}
$$

Let us now average (112) and (124) over $\ell_{2} \in\left\{1, \ldots, L_{2}\right\}$ weighted by $p_{I}\left(\ell_{2}\right)$. We thus have

$$
\begin{aligned}
& n(R(\boldsymbol{D}, \boldsymbol{\epsilon}, C)+C) \\
& \geq n \sum_{\ell_{2}} P_{I}\left(\ell_{2}\right) I\left(\hat{X}_{\ell_{1}, Q}, X_{\ell_{2}, Q}, U_{\ell_{2}, Q}, Q ; \boldsymbol{X}_{Q}\right)-n \delta_{1}(\epsilon) \\
& =n I\left(\hat{X}_{\ell_{1}, Q}, X_{I, Q}, U_{I, Q}, Q ; \boldsymbol{X}_{Q} \mid I\right)-n \delta_{1}(\epsilon)
\end{aligned}
$$

and similarly, we have

$$
\begin{aligned}
& n R(\boldsymbol{D}, \boldsymbol{\epsilon}, C) \\
& \geq n I\left(X_{I, Q} ; U_{I, Q}, Q \mid I\right) \\
& \quad+n I\left(\hat{X}_{\ell_{1}, Q} ; \boldsymbol{X}_{Q} \mid U_{I, Q}, Q, X_{I, Q}, I\right)-n \delta_{2}(\epsilon)
\end{aligned}
$$

Finally, we verify that 28a holds. Since the rate-distortion-memory $(R, D, C)$ is admissible, for any $\epsilon^{\prime}>0$ we have

$$
\begin{aligned}
\left(D+\epsilon^{\prime}\right) & \geq \mathbb{E}\left[\bar{d}_{\ell_{1}}\left(X_{\ell_{1}}^{n}, \hat{X}_{\ell_{1}}^{n}\right)\right] \\
& =\frac{1}{n} \sum_{i=1}^{n} \mathbb{E}\left[d_{\ell_{1}}\left(X_{\ell_{1}, i}, \hat{X}_{\ell_{1}, i}\right)\right] \\
& =\mathbb{E}\left[d_{\ell_{1}}\left(X_{\ell_{1}, Q}, \hat{X}_{\ell_{1}, Q}\right)\right]
\end{aligned}
$$

We now define $U=\left(U_{I, Q}, Q\right)$, and rename $\hat{X}_{\ell_{1}, Q}, X_{I, Q}$, and $\boldsymbol{X}_{Q}$ to $\hat{X}_{\ell_{1}}, X_{I}$, and $\boldsymbol{X}$, respectively. It is not difficult to see that 41 holds.

\section{REFERENCES}

[1] M. A. Maddah-Ali and U. Niesen, "Fundamental limits of caching," IEEE Transactions on Information Theory, vol. 60, no. 5, pp. 2856 - 2867, 2014.

[2] U. Niesen and M. A. Maddah-Ali, "Coded caching with nonuniform demands," arXiv, 1308.0178, 2013.

[3] Y. Shkel and S. Verdú, "A coding theorem for f-separable distortion functions," in proceedings Information Theory and Applications Workshop (ITA), San Diego, USA, 2016.

[4] V. Tikhomirov, "On the notion of mean," Selected works of A. N. Kolmogorov series Mathematics and its Applications, vol. $25,1991$.

[5] K. Visweswariah, S. R. Kulkarni, and S. Verdú, "Output distribution of the Burrows-Wheeler transform," in proceedings IEEE International Symposium on Information Theory, Sorrento, Italy, 2000.

[6] M. Effros, K. Visweswariah, S. R. Kulkarni and S. Verdú, "Universal lossless source coding with the Burrows Wheeler transform," IEEE Transactions on Information Theory, vol. 48, no. 5, 2002.

[7] C. Y. Wang, S. H. Lim and M. Gastpar, "Information-theoretic caching: sequential coding for computing," arXiv, vol. 1504.00553, 2015.

[8] P. Hassanzadeh, E. Erkip, J. Llorca and A. Tulino, "Distortion-memory tradeoffs in cache-aided wireless video delivery," arXiv, 1511.03932, 2015.

[9] Q. Yang and D. Gündüz, "Centralized coded caching for Heterogenous lossy requests," arXiv, 1604.08178, 2016.

[10] R. Gray, "Conditional rate-distortion theory," Stanford University Technical Report, October, 1972.

[11] K. B. Viswanatha, E. Akyol and K. Rose, "The lossy common information of correlated sources," IEEE Transactions on Information Theory, vol. 60, no. 6 , pp. $3238-3253,2014$.

[12] P. Gács and J. Körner, "Common information is far less than mutual information," Problems of Control and Information Theory, vol. 2, no. 2, p. 149 $162,1973$.

[13] A. Wyner, "The common information of two dependent random variables," IEEE Transactions on Information Theory, vol. 21, no. 2, pp 163 - 179, 1975. 
[14] R. Gray and A. Wyner, "Source coding for a simple network," Bell Systems Technical Journal, vol. 53, no. 9, pp. 1681 - $1721,1974$.

[15] J. C. Kieffer, "Sample converses in source coding theory," IEEE Transactions on Information Theory, vol. 37, no. 2, pp. 263 - 268, 1991.

[16] A. Wyner, "The rate-distortion function for source coding with side-information at the decoder-II: general sources," Information and Control, vol. 38, pp. $60-80,1978$.

[17] G. Xu, W. Liu, and B. Chen, "Wyner's common information for continuous random variables - a lossy source coding interpretation," in proceedings 45th Annual Conference on Information Sciences and Systems (CISS), Baltimore, USA, 2011.

[18] A. Lapidoth and S. Tinguely, "Sending a bivariate Gaussian over a Gaussian MAC," IEEE Transactions on Information Theory, vol. 56, no. 6, pp. 2714 $-2752,2010$.

[19] R. Timo, A. Grant, and G. Kramer, "Lossy broadcasting with complementary side information," IEEE Transactions on Information Theory, vol. 59, no. 1 , pp. $104-131,2013$.

[20] A. Wyner and J. Ziv, "The rate-distortion function for source coding with side information at the decoder," IEEE Transactions on Information Theory, vol. 22 , no. 1 , pp. $1-10,1976$.

[21] E. Tuncel, "Slepian-Wolf coding over broadcast channels," IEEE Transactions on Information Theory, vol. 52, no. 4 , pp. 1469 - 1482, 2006.

[22] A. El Gamal and Y.-H. Kim, Network Information Theory, Cambridge University Press, 2011.

[23] B. N. Vellambi and R. Timo, "Multi-terminal source coding: Can zero-rate encoders enlarge the rate region?," in proceedings International Zurich Seminar on Communications (IZS), Zurich, Switzerland, 2010.

[24] G. Kramer, "Topics in multi-user information theory," Foundations and Trends in Communications and Information Theory, vol. 4, no. 45, pp. 265 444, 2008.

[25] T. Cover and J. Thomas, Elements of Information Theory, John Wiley and Sons, 2006.

[26] V. M. Prabhakaran and M. M. Prabhakaran, "Assisted common information with an application to secure two-party sampling," IEEE Transactions on Information Theory, vol. 60, no. 6, pp. 3413 - 3434, 2014.

[27] R. Gray, "A new class of lower bounds to information rates of stationary sources via conditional rate-distortion functions," IEEE Transactions on Information Theory, vol. 19, no. 4, pp. 480 - 489, 1973.

[28] S. Watanabe, "Second-order region for Gray-Wyner network," arXiv, 1508.04227, 2015.

[29] W. H. Gu and M. Effros, "A strong converse for a collection of network source coding problems," in proceedings IEEE International Symposium on Information Theory, Seoul, South Korea, 2009.

[30] R. W. Yeung, Information theory and network coding, Springer, 2008.

[31] C. Y. Wang, S. H. Lim and M. Gastpar, "A New Converse Bound for Coded Caching," arXiv, vol. 1601.05690, 2016.

[32] S. Saeedi Bidokhti, M. Wigger and R. Timo, "Noisy Broadcast Networks with Receiver Caching," arXiv, vol. 1605.02317, 2016.

[33] S. Saeedi Bidokhti, M. Wigger and R. Timo, "An Upper Bound on the Capacity-Memory Tradeoff of Degraded Broadcast Channels," in proceedings International Symposium on Turbo Codes \& Iterative Information Processing, Brest, France, 2016. 